\title{
A bis(thiophosphinoyl)methylene ruthenium carbene complex: Synthesis, X-ray crystal structure and DFT calculations of its thermally promoted reverse $\alpha$-hydride migration process
}

Thibault Cantat, ${ }^{\mathrm{a}}$ Matthieu Demange, ${ }^{\mathrm{a}}$ Nicolas Mézailles, ${ }^{\mathrm{a}}$ Louis Ricard, ${ }^{\mathrm{a}}$ Yves Jean ${ }^{\mathrm{a}}$ and Pascal Le Floch ${ }^{\text {a* }}$

\footnotetext{
${ }^{a}$ Laboratoire « Hétéroéléments et Coordination », UMR CNRS 7653 (DCPH), Département de Chimie, Ecole Polytechnique, 91128 Palaiseau Cédex, France

Tel: +33169334570; Fax: +3316933 3990

E-mail: lefloch@poly.polytechnique.fr
} 


\section{Crystallographic data for 2}

Figure S1: Molecular structure of $\mathbf{2}$

Table S1-1: Crystal data and structure refinement for $\mathbf{2}$

1 page

1 page

Table S1-2: Atomic Coordinates and equivalent isotropic displacement parameters for 2

Table S1-3: Bond lengths $(\AA)$ and angles $\left({ }^{\circ}\right)$ for 2

1 page

4 pages

Table S1-4: Anisotropic displacement parameters for $\mathbf{2}$

2 pages

Table S1-5: Coordinates and equivalent isotropic

displacement parameters for $\mathbf{2}$

1 page

Crystallographic data for 3

Figure S2: Molecular structure of $\mathbf{3}(\mathrm{S}, \mathrm{S})$

Table S2-1: Crystal data and structure refinement for $\mathbf{3}$

Table S2-2: Atomic Coordinates and equivalent isotropic displacement parameters for $\mathbf{3}$

Table S2-3: Bond lengths $(\AA)$ and angles $\left(^{\circ}\right)$ for 3

Table S2-4: Anisotropic displacement parameters for 3

Table S2-5: Coordinates and equivalent isotropic

displacement parameters for $\mathbf{3}$

1 page

1 page

3 pages

7 pages

3 pages

2 pages

Theoretical data for the conversion of 2' in 3'

Computational details

1 page

Figure S3-1: Molecular structure of 2'

1 page

Table S3-1: Coordinates for 2'TS4'

Figure S3-2: Molecular structure of 2'TS4'

Table S3-2: Coordinates for 2'TS4'

Figure S3-3: Molecular structure of 4'

Table S3-3: Coordinates for 4'

Figure S3-4: Molecular structure of 4'TS5'

Table S3-4: Coordinates for 4'TS5'

Figure S3-5: Molecular structure of 5,

Table S3-5: Coordinates for 5'

Figure S3-6: Molecular structure of 5'TS6'

1 page

1 page

1 page

1 page

1 page

1 page

1 page

1 page

1 page

1 page

Table S3-6: Coordinates for 5'TS6'

Figure S3-7: Molecular structure of 6'

1 page

1 page

Table S3-7: Coordinates for 6'

1 page

Figure S3-8: Molecular structure of 6'TS3'

1 page

Table S3-8: Coordinates for 6'TS3'

Figure S3-9: Molecular structure of 3'

1 page

Table S3-9: Coordinates for 3'

1 page

1 page 
Figure S1: Molecular structure of 2

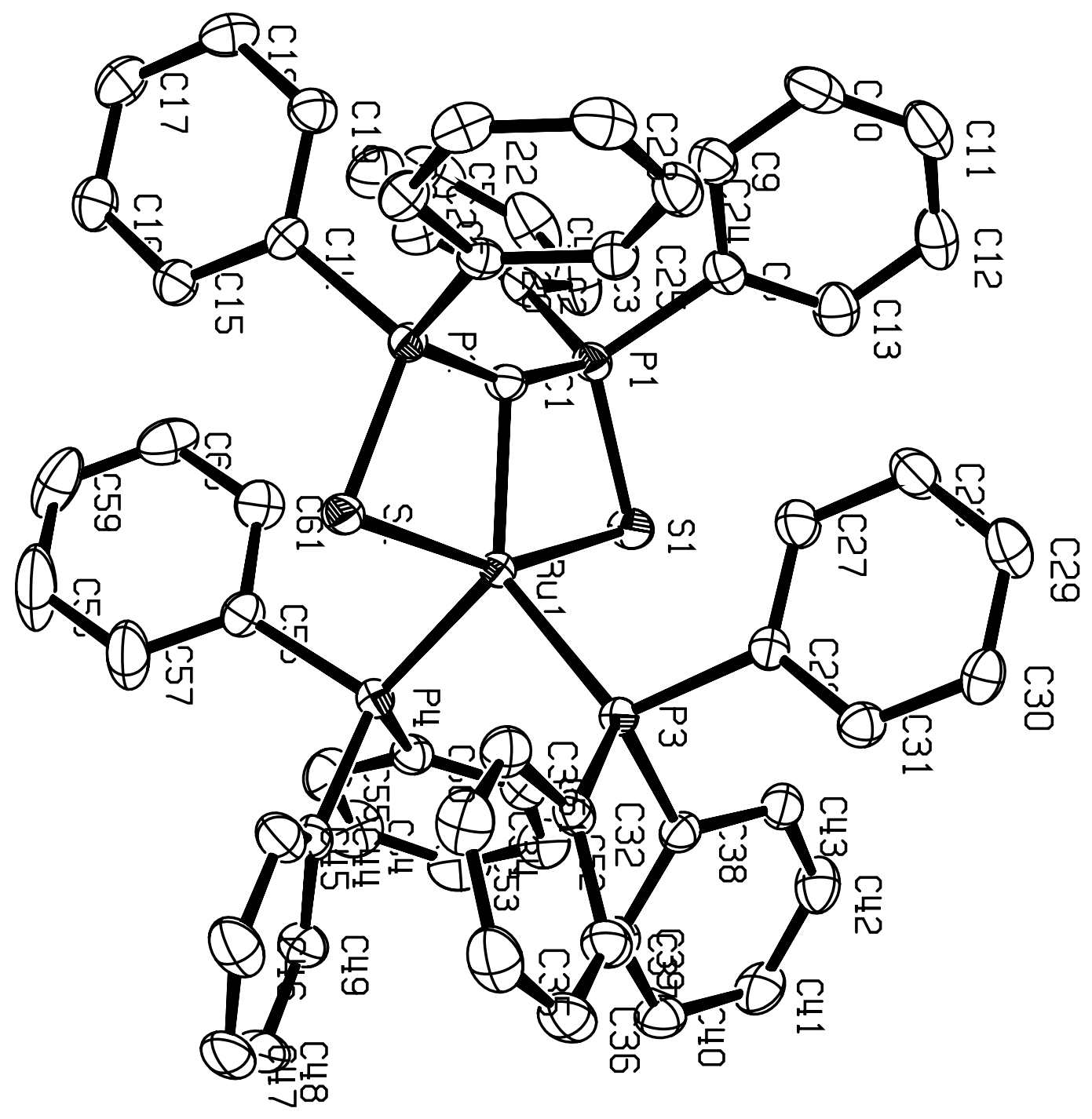


Table S1-1. Crystal data for 2

Compound

Molecular formula

Molecular weight

Crystal habit

Crystal dimensions(mm)

Crystal system

Space group

$\mathrm{a}(\AA)$

$\mathrm{b}(\AA)$

$c(\AA)$

$\alpha\left(^{\circ}\right)$

$\beta\left(^{\circ}\right)$

$\gamma\left({ }^{\circ}\right)$

$\mathrm{V}\left(\AA^{3}\right)$

$\mathrm{Z}$

$\mathrm{d}\left(\mathrm{g}-\mathrm{cm}^{-3}\right)$

$\mathrm{F}(000)$

$\mu\left(\mathrm{cm}^{-1}\right)$

Absorption corrections

Diffractometer

$\mathrm{X}$-ray source

$\lambda(\AA)$

Monochromator

$\mathrm{T}(\mathrm{K})$

Scan mode

Maximum $\theta$

HKL ranges

Reflections measured

Unique data

Rint

Reflections used

Criterion

Refinement type

Hydrogen atoms

Parameters refined

Reflections / parameter

wR2

R1

Weights $a, b$

GoF

difference peak / hole (e $\left.\AA^{-3}\right)$ rucarb

$\mathrm{C}_{61} \mathrm{H}_{50} \mathrm{P}_{4} \mathrm{RuS} \mathrm{S}_{2}$

1072.08

dark red needle

$0.22 \times 0.08 \times 0.08$

monoclinic

$\mathrm{P} 2{ }_{1} / \mathrm{c}$

18.4040(10)

$16.1150(10)$

17.3300(10)

90.00

104.5900(10)

90.00

4974.0(5)

4

1.432

2208

0.569

multi-scan ; 0.8850 min, 0.9559 max

KappaCCD

$\mathrm{MoK} \alpha$

0.71069

graphite

150.0(1)

phi and omega scans

30.02

$-2525 ;-2220 ;-2424$

23879

14470

0.0250

10950

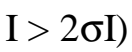

Fsqd

mixed

613

17

0.0902

0.0350

$0.0414 ; 0.1081$

1.018

$0.523(0.074) /-0.807(0.074)$ 
Table S1-2: Atomic Coordinates and equivalent isotropic displacement parameters for 2 Atomic Coordinates $\left(A \times 10^{\wedge} 4\right)$ and equivalent isotropic
displacement parameters $\left(A^{\wedge} 2 \times 10^{\wedge} 3\right)$ for rucarb

\begin{tabular}{|c|c|c|c|c|}
\hline atom & $\mathrm{x}$ & $\mathrm{Y}$ & $z$ & $\mathrm{U}(\mathrm{eq})$ \\
\hline \multicolumn{5}{|c|}{----------------} \\
\hline $\mathrm{Ru}(1)$ & $2554(1)$ & $-4943(1)$ & $2593(1)$ & $17(1)$ \\
\hline$S(1)$ & $1385(1)$ & $-5757(1)$ & $2423(1)$ & $24(1)$ \\
\hline$S(2)$ & $3473(1)$ & $-3845(1)$ & $3187(1)$ & $22(1)$ \\
\hline $\mathrm{P}(1)$ & $1202(1)$ & $-4823(1)$ & $3136(1)$ & $20(1)$ \\
\hline$P(2)$ & $2582(1)$ & $-3478(1)$ & $3585(1)$ & $18(1)$ \\
\hline P (3) & 2558 (1) & $-4941(1)$ & $1287(1)$ & $18(1)$ \\
\hline P (4) & $3382(1)$ & $-6001(1)$ & $2947(1)$ & $20(1)$ \\
\hline$C(1)$ & $1971(1)$ & $-4239(1)$ & $3222(1)$ & $20(1)$ \\
\hline$C(2)$ & $1053(1)$ & $-5231(1)$ & $4062(1)$ & $24(1)$ \\
\hline$C(3)$ & 499 (1) & $-5823(2)$ & $4032(1)$ & $37(1)$ \\
\hline$C(4)$ & $349(1)$ & $-6106(2)$ & $4732(2)$ & $47(1)$ \\
\hline$C(5)$ & 759 (1) & $-5813(2)$ & $5457(2)$ & $44(1)$ \\
\hline$C(6)$ & $1322(1)$ & $-5239(2)$ & 5491 (1) & $40(1)$ \\
\hline$C(7)$ & 1465 (1) & $-4944(1)$ & $4787(1)$ & $33(1)$ \\
\hline$C(8)$ & $335(1)$ & $-4256(1)$ & $2710(1)$ & $23(1)$ \\
\hline$C(9)$ & $239(1)$ & $-3479(1)$ & 3025 (1) & $30(1)$ \\
\hline$C(10)$ & $-389(1)$ & $-3005(2)$ & $2698(1)$ & $38(1)$ \\
\hline$C(11)$ & $-935(1)$ & $-3314(2)$ & 2059 (1) & $39(1)$ \\
\hline C (12) & $-852(1)$ & $-4084(2)$ & $1752(1)$ & $37(1)$ \\
\hline C (13) & $-212(1)$ & $-4557(1)$ & 2069 (1) & $32(1)$ \\
\hline$C(14)$ & $2797(1)$ & $-3352(1)$ & $4657(1)$ & $20(1)$ \\
\hline$C(15)$ & $3395(1)$ & $-3766(1)$ & $5158(1)$ & $26(1)$ \\
\hline$C(16)$ & 3487 (1) & $-3743(1)$ & 5976 (1) & $33(1)$ \\
\hline C (17) & 2989 (1) & $-3311(1)$ & $6298(1)$ & $33(1)$ \\
\hline C (18) & 2395 (1) & $-2888(1)$ & $5804(1)$ & $31(1)$ \\
\hline C (19) & 2297 (1) & $-2905(1)$ & 4989 (1) & $26(1)$ \\
\hline$C(20)$ & 2277 (1) & $-2449(1)$ & $3209(1)$ & $21(1)$ \\
\hline C (21) & $2666(1)$ & $-1746(1)$ & $3548(1)$ & $25(1)$ \\
\hline C (22) & 2438 (1) & $-971(1)$ & 3249 (1) & $28(1)$ \\
\hline C (23) & $1817(1)$ & $-883(1)$ & $2611(1)$ & $32(1)$ \\
\hline$C(24)$ & 1429 (1) & $-1577(1)$ & 2267 (1) & $31(1)$ \\
\hline$C(25)$ & $1657(1)$ & $-2358(1)$ & $2564(1)$ & $25(1)$ \\
\hline$C(26)$ & $1753(1)$ & $-4334(1)$ & $693(1)$ & $19(1)$ \\
\hline C (27) & 1205 (1) & $-4019(1)$ & $1031(1)$ & $27(1)$ \\
\hline C (28) & 589 (1) & $-3600(2)$ & $572(1)$ & $35(1)$ \\
\hline C (29) & $507(1)$ & $-3491(1)$ & $-237(1)$ & $32(1)$ \\
\hline$C(30)$ & 1048 (1) & $-3804(1)$ & $-587(1)$ & $27(1)$ \\
\hline C (31) & $1667(1)$ & $-4219(1)$ & $-121(1)$ & $24(1)$ \\
\hline C (32) & $3323(1)$ & $-4471(1)$ & $911(1)$ & $22(1)$ \\
\hline C (33) & 3647 (1) & $-3743(1)$ & $1270(1)$ & $27(1)$ \\
\hline C (34) & $4220(1)$ & $-3347(1)$ & $1012(1)$ & $35(1)$ \\
\hline$C(35)$ & 4478 (1) & $-3677(2)$ & 395 (1) & $37(1)$ \\
\hline$C(36)$ & $4152(1)$ & $-4391(2)$ & 27 (1) & $33(1)$ \\
\hline$C(37)$ & $3575(1)$ & $-4782(1)$ & $270(1)$ & $28(1)$ \\
\hline C (38) & 2388 (1) & $-5924(1)$ & $739(1)$ & $22(1)$ \\
\hline C (39) & $2979(1)$ & $-6449(1)$ & $697(1)$ & $25(1)$ \\
\hline$C(40)$ & 2846 (1) & $-7211(1)$ & $319(1)$ & $31(1)$ \\
\hline$C(41)$ & 2116 (1) & $-7480(1)$ & $9(1)$ & $35(1)$ \\
\hline$C(42)$ & $1529(1)$ & $-6983(1)$ & $75(1)$ & $33(1)$ \\
\hline$C(43)$ & $1655(1)$ & $-6204(1)$ & 427 (1) & $26(1)$ \\
\hline C ( 4 4) & 4238 (1) & $-6069(1)$ & 2579 (1) & $25(1)$ \\
\hline$C(45)$ & $4599(1)$ & $-5333(1)$ & 2479 (1) & $31(1)$ \\
\hline$C(46)$ & $5222(1)$ & $-5336(2)$ & $2167(1)$ & $40(1)$ \\
\hline$C(47)$ & $5490(1)$ & $-6072(2)$ & $1948(1)$ & $41(1)$ \\
\hline$C(48)$ & $5147(1)$ & $-6807(2)$ & $2042(1)$ & $44(1)$ \\
\hline C (49) & $4523(1)$ & $-6812(2)$ & $2356(1)$ & $34(1)$ \\
\hline$C(50)$ & $3091(1)$ & $-7098(1)$ & $2864(1)$ & $24(1)$ \\
\hline C (51) & $2550(1)$ & $-7377(1)$ & $2202(1)$ & $26(1)$ \\
\hline C (52) & 2416 (1) & $-8217(1)$ & $2064(1)$ & $33(1)$ \\
\hline C (53) & $2811(1)$ & $-8792(1)$ & $2595(2)$ & $40(1)$ \\
\hline C (54) & $3323(1)$ & $-8531(1)$ & $3282(2)$ & $45(1)$ \\
\hline
\end{tabular}




$\begin{array}{lllll}C(55) & 3464(1) & -7693(1) & 3415(1) & 37(1) \\ C(56) & 3745(1) & -5934(1) & 4031(1) & 25(1) \\ C(57) & 4489(1) & -6026(2) & 4434(1) & 62(1) \\ C(58) & 4706(1) & -6012(3) & 5264(2) & 82(1) \\ C(59) & 4191(1) & -5919(2) & 5698(1) & 49(1) \\ C(60) & 3453(1) & -5823(1) & 5313(1) & 36(1) \\ C(61) & 3232(1) & -5827(1) & 4490(1) & 30(1)\end{array}$

$\mathrm{U}(\mathrm{eq})$ is defined as $1 / 3$ the trace of the Uij tensor. 
Table S1-3: Bond lengths $(\AA)$ and angles $\left({ }^{\circ}\right)$ for 2

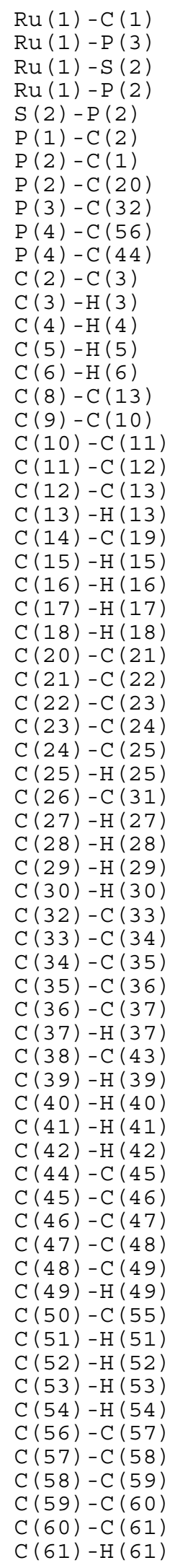

$2.053(2)$

$2.2656(5$

$2.4843(5$

2. $9130(5$

$2.0206(6$

$1.818(2)$

$1.676(2)$

$1.817(2)$

$1.855(2)$

$1.833(2)$

$1.845(2)$

1. 388 (3)

0.9500

0.9500

0.9500

0.9500

1. 385 (3)

1. $383(3)$

1. $387(3)$

1. $374(3)$

1.395 (3)

0.9500

$1.403(3)$

0.9500

0.9500

0.9500

0.9500

1.389(3)

$1.378(3)$

$1.383(3)$

$1.377(3)$

1. $385(3)$

0.9500

1.390(3)

0.9500

0.9500

0.9500

0.9500

1.391(3)

1.398(3)

$1.382(3)$

1. $378(3)$

1.389(3)

0.9500

1.397(2)

0.9500

0.9500

0.9500

0.9500

1. 392 (3)

$1.385(3)$

$1.373(3)$

$1.371(3)$

1. $389(3)$

0.9500

1. 404 (3)

0.9500

0.9500

0.9500

0.9500

$1.378(3)$

1. $392(3)$

$1.359(3)$

$1.363(3)$

1.381(3)

0.9500
$\mathrm{Ru}(1)-\mathrm{P}(4)$

$\mathrm{Ru}(1)-\mathrm{S}(1)$

$\mathrm{Ru}(1)-\mathrm{P}(1)$

$S(1)-P(1)$

$P(1)-C(1)$

$P(1)-C(8)$

$P(2)-C(14)$

$\mathrm{P}(3)-\mathrm{C}(38)$

$P(3)-C(26)$

$P(4)-C(50)$

$C(2)-C(7)$

$C(3)-C(4)$

$C(4)-C(5)$

$C(5)-C(6)$

C (6) $-C(7)$

$\mathrm{C}(7)-\mathrm{H}(7)$

C (8) $-\mathrm{C}(9)$

$\mathrm{C}(9)-\mathrm{H}(9)$

$\mathrm{C}(10)-\mathrm{H}(10)$

$\mathrm{C}(11)-\mathrm{H}(11)$

$\mathrm{C}(12)-\mathrm{H}(12)$

C (14)-C (15)

C (15) - C (16)

C (16) - C (17)

C (17) - C (18)

C (18)-C (19)

$\mathrm{C}(19)-\mathrm{H}(19)$

$C(20)-C(25)$

$\mathrm{C}(21)-\mathrm{H}(21)$

$\mathrm{C}(22)-\mathrm{H}(22)$

$\mathrm{C}(23)-\mathrm{H}(23)$

$\mathrm{C}(24)-\mathrm{H}(24)$

$C(26)-C(27)$

$C(27)-C(28)$

$C(28)-C(29)$

$C(29)-C(30)$

$C(30)-C(31)$

$\mathrm{C}(31)-\mathrm{H}(31)$

C (32) $-\mathrm{C}(37)$

$\mathrm{C}(33)-\mathrm{H}(33)$

$\mathrm{C}(34)-\mathrm{H}(34)$

$\mathrm{C}(35)-\mathrm{H}(35)$

$\mathrm{C}(36)-\mathrm{H}(36)$

C (38) - C (39)

C (39) $-C(40)$

$C(40)-C(41)$

$C(41)-C(42)$

C ( 42$)-C(43)$

$\mathrm{C}(43)-\mathrm{H}(43)$

C (44)-C (49)

$\mathrm{C}(45)-\mathrm{H}(45)$

$\mathrm{C}(46)-\mathrm{H}(46)$

$\mathrm{C}(47)-\mathrm{H}(47)$

$\mathrm{C}(48)-\mathrm{H}(48)$

C ( 50$)-C(51)$

$C(51)-C(52)$

$C(52)-C(53)$

$C(53)-C(54)$

C (54) $-C(55)$

$\mathrm{C}(55)-\mathrm{H}(55)$

C (56)-C (61)

$\mathrm{C}(57)-\mathrm{H}(57)$

C (58) $-\mathrm{H}(58)$

$\mathrm{C}(59)-\mathrm{H}(59)$

$\mathrm{C}(60)-\mathrm{H}(60)$
2. 2641 (5)

$2.4739(5)$

$2.8803(5)$

$2.0269(7)$

1.675 (2)

$1.826(2)$

$1.810(2)$

$1.833(2)$

$1.856(2)$

$1.843(2)$

$1.375(3)$

$1.386(3)$

$1.376(4)$

$1.379(3)$

1.394(3)

0.9500

1.395 (3)

0.9500

0.9500

0.9500

0.9500

1. 389 (3)

$1.386(3)$

$1.375(3)$

$1.386(3)$

$1.378(3)$

0.9500

1.391 (3)

0.9500

0.9500

0.9500

0.9500

1. $384(2)$

$1.386(3)$

$1.383(3)$

$1.385(3)$

1.390(3)

0.9500

1. 400 (3)

0.9500

0.9500

0.9500

0.9500

1.393(3)

1.384(3)

$1.384(3)$

$1.374(3)$

1. 389 (3)

0.9500

1. 401 (3)

0.9500

0.9500

0.9500

0.9500

1.391(3)

$1.387(3)$

$1.377(3)$

$1.384(3)$

$1.383(3)$

0.9500

1.390(3)

0.9500

0.9500

0.9500

0.9500 


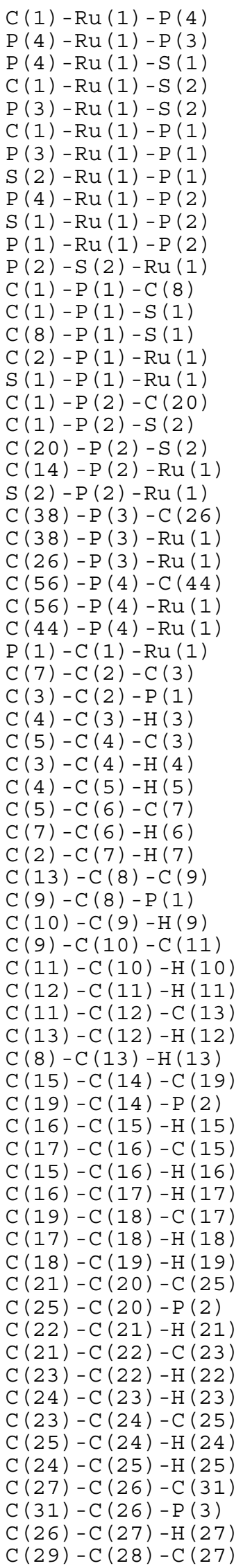

\begin{tabular}{|c|c|}
\hline $132.71(5)$ & $C(1)-R u(1)-P(3)$ \\
\hline $95.75(2)$ & $C(1)-R u(1)-S(1)$ \\
\hline $98.02(2)$ & $\mathrm{P}(3)-\operatorname{Ru}(1)-\mathrm{S}(1)$ \\
\hline $77.22(5)$ & $\mathrm{P}(4)-\mathrm{Ru}(1)-\mathrm{S}(2)$ \\
\hline $103.87(2)$ & $S(1)-R u(1)-S(2)$ \\
\hline $34.84(5)$ & $\mathrm{P}(4)-\mathrm{Ru}(1)-\mathrm{P}(1)$ \\
\hline $23.12(2)$ & $S(1)-R u(1)-P(1)$ \\
\hline $11.76(2)$ & $C(1)-\operatorname{Ru}(1)-P(2)$ \\
\hline $22.68(2)$ & $\mathrm{P}(3)-\mathrm{Ru}(1)-\mathrm{P}(2)$ \\
\hline $12.63(2)$ & $S(2)-R u(1)-P(2)$ \\
\hline $8.96(1)$ & $\mathrm{P}(1)-\mathrm{S}(1)-\mathrm{Ru}(1)$ \\
\hline $9.85(2)$ & $C(1)-P(1)-C(2)$ \\
\hline $12.8(1)$ & $C(2)-P(1)-C(8)$ \\
\hline $01.84(6)$ & $C(2)-P(1)-S(1)$ \\
\hline $12.80(7)$ & $\mathrm{C}(1)-\mathrm{P}(1)-\mathrm{Ru}(1)$ \\
\hline $126.80(6)$ & $C(8)-P(1)-R u(1)$ \\
\hline $57.44(2)$ & $C(1)-P(2)-C(14)$ \\
\hline $114.6(1)$ & $C(14)-P(2)-C(20)$ \\
\hline $100.56(6)$ & $C(14)-P(2)-S(2)$ \\
\hline $110.41(6)$ & $\mathrm{C}(1)-\mathrm{P}(2)-\mathrm{Ru}(1)$ \\
\hline $31.54(6)$ & $C(20)-P(2)-R u(1)$ \\
\hline $7.09(2)$ & $C(38)-P(3)-C(32)$ \\
\hline $.20(8)$ & $C(32)-P(3)-C(26)$ \\
\hline $18.24(6)$ & $\mathrm{C}(32)-\mathrm{P}(3)-\mathrm{Ru}(1)$ \\
\hline $10.63(6)$ & $C(56)-P(4)-C(50)$ \\
\hline $3.56(8)$ & $C(50)-P(4)-C(44)$ \\
\hline $6.27(6)$ & $\mathrm{C}(50)-\mathrm{P}(4)-\mathrm{Ru}(1)$ \\
\hline $1.76(6)$ & $P(1)-C(1)-P(2)$ \\
\hline $100.7(1)$ & $\mathrm{P}(2)-\mathrm{C}(1)-\mathrm{Ru}(1)$ \\
\hline $119.8(2)$ & $C(7)-C(2)-P(1)$ \\
\hline $119.2(2)$ & $C(4)-C(3)-C(2)$ \\
\hline 20.0 & $\mathrm{C}(2)-\mathrm{C}(3)-\mathrm{H}(3)$ \\
\hline $120.1(2)$ & $\mathrm{C}(5)-\mathrm{C}(4)-\mathrm{H}(4)$ \\
\hline 9.9 & $C(4)-C(5)-C(6)$ \\
\hline 9.9 & $\mathrm{C}(6)-\mathrm{C}(5)-\mathrm{H}(5)$ \\
\hline $9.8(2)$ & $\mathrm{C}(5)-\mathrm{C}(6)-\mathrm{H}(6)$ \\
\hline .1 & $C(2)-C(7)-C(6)$ \\
\hline 9.9 & $\mathrm{C}(6)-\mathrm{C}(7)-\mathrm{H}(7)$ \\
\hline $9.4(2)$ & $C(13)-C(8)-P(1)$ \\
\hline $8.3(1)$ & $C(10)-C(9)-C(8)$ \\
\hline 7 & $\mathrm{C}(8)-\mathrm{C}(9)-\mathrm{H}(9)$ \\
\hline $119.5(2)$ & $\mathrm{C}(9)-\mathrm{C}(10)-\mathrm{H}(10)$ \\
\hline 120.2 & $C(12)-C(11)-C(10)$ \\
\hline-19.9 & $\mathrm{C}(10)-\mathrm{C}(11)-\mathrm{H}(11)$ \\
\hline $20.5(2)$ & $\mathrm{C}(11)-\mathrm{C}(12)-\mathrm{H}(12)$ \\
\hline 9.8 & $C(8)-C(13)-C(12)$ \\
\hline 0.2 & $\mathrm{C}(12)-\mathrm{C}(13)-\mathrm{H}(13)$ \\
\hline $9.3(2)$ & $C(15)-C(14)-P(2)$ \\
\hline $8.8(1)$ & $C(16)-C(15)-C(14)$ \\
\hline .0 & $\mathrm{C}(14)-\mathrm{C}(15)-\mathrm{H}(15)$ \\
\hline $.4(2)$ & $\mathrm{C}(17)-\mathrm{C}(16)-\mathrm{H}(16)$ \\
\hline .8 & $C(16)-C(17)-C(18)$ \\
\hline 0 & $\mathrm{C}(18)-\mathrm{C}(17)-\mathrm{H}(17)$ \\
\hline $20.2(2)$ & $\mathrm{C}(19)-\mathrm{C}(18)-\mathrm{H}(18)$ \\
\hline 19.9 & $C(18)-C(19)-C(14)$ \\
\hline 20.0 & $\mathrm{C}(14)-\mathrm{C}(19)-\mathrm{H}(19)$ \\
\hline $9.0(2)$ & $C(21)-C(20)-P(2)$ \\
\hline $0.1(2)$ & $C(22)-C(21)-C(20)$ \\
\hline 9.8 & $\mathrm{C}(20)-\mathrm{C}(21)-\mathrm{H}(21)$ \\
\hline $.3(2)$ & $\mathrm{C}(21)-\mathrm{C}(22)-\mathrm{H}(22)$ \\
\hline 9.8 & $C(24)-C(23)-C(22)$ \\
\hline 0.1 & $\mathrm{C}(22)-\mathrm{C}(23)-\mathrm{H}(23)$ \\
\hline $.2(2)$ & $\mathrm{C}(23)-\mathrm{C}(24)-\mathrm{H}(24)$ \\
\hline .9 & $C(24)-C(25)-C(20)$ \\
\hline 119.8 & $\mathrm{C}(20)-\mathrm{C}(25)-\mathrm{H}(25)$ \\
\hline $8.1(2)$ & $C(27)-C(26)-P(3)$ \\
\hline $0.3(1)$ & $C(26)-C(27)-C(28)$ \\
\hline .5 & $\mathrm{C}(28)-\mathrm{C}(27)-\mathrm{H}(27)$ \\
\hline 5 & $\mathrm{C}(29)-\mathrm{C}(28)-\mathrm{H}(28)$ \\
\hline
\end{tabular}

$131.53(5)$

$78.47(5)$

$95.94(2)$

$94.77(2)$

$155.18(2)$

$122.68(2)$

$43.67(2)$

$34.20(5)$

$125.75(2)$

43.06 (1)

$78.89(2)$

$115.9(1)$

$103.10(8)$

$110.79(7)$

$44.47(6)$

$129.86(6)$

$114.85(8)$

$103.46(8)$

$113.34(6)$

$43.54(6)$

$124.77(6)$

$102.49(8)$

$99.21(8)$

$123.05(6)$

$99.2(1)$

$99.9(1)$

$122.60(6)$

$156.5(1)$

$102.26(8)$

$120.9(2)$

$120.0(2)$

120.0

119.9

$120.2(2)$

119.9

120.1

$120.2(2)$

119.9

$122.2(2)$

$120.6(2)$

119.7

120.2

$120.3(2)$

119.9

119.8

$119.7(2)$

120.2

$121.5(1)$

120.1 (2)

120.0

119.8

$120.1(2)$

120.0

119.9

$120.0(2)$

120.0

$120.9(1)$

$120.4(2)$

119.8

119.8

$119.8(2)$

120.1

119.9

$120.3(2)$

119.8

$121.5(1)$

$120.9(2)$

119.5

119.7 


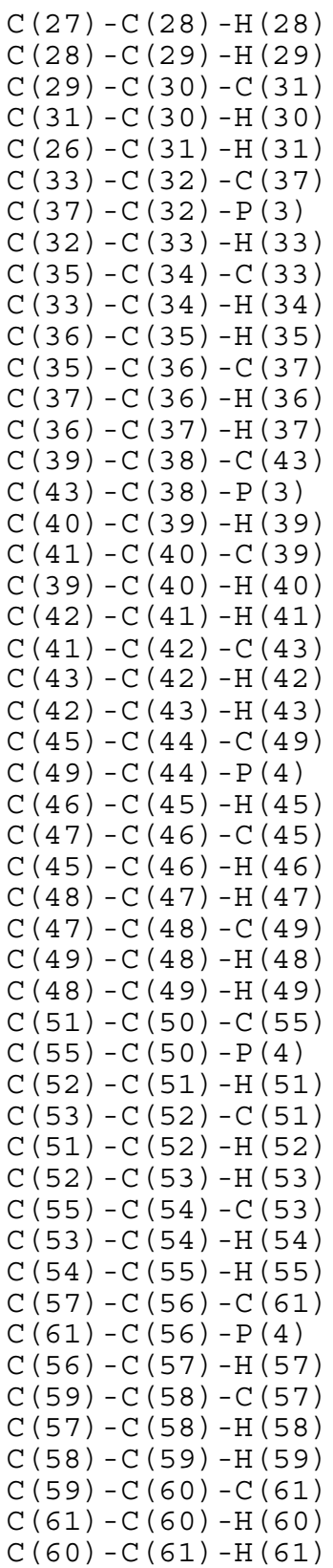

119.7
120.3
$119.6(2)$
120.2
119.3
$117.8(2)$
$124.4(2)$
119.5
$120.4(2)$
119.8
120.5
$121.1(2)$
119.5
119.7
$118.3(2)$
$120.1(1)$
119.5
$120.1(2)$
120.0
120.3
$121.0(2)$
119.5
120.0
$118.1(2)$
$123.9(2)$
119.5
$120.0(2)$
120.0
119.8
$120.2(2)$
119.9
119.8
$117.8(2)$
$121.3(2)$
119.5
$120.1(2)$
120.0
120.0
$119.9(2)$
120.1
119.5
$117.0(2)$
$117.9(1)$
119.6
$120.9(2)$
119.5
120.4
$120.2(2)$
119.9
119.2

$C(28)-C(29)-C(30)$

$\mathrm{C}(30)-\mathrm{C}(29)-\mathrm{H}(29)$

$\mathrm{C}(29)-\mathrm{C}(30)-\mathrm{H}(30)$

$C(26)-C(31)-C(30)$

$\mathrm{C}(30)-\mathrm{C}(31)-\mathrm{H}(31)$

$\mathrm{C}(33)-\mathrm{C}(32)-\mathrm{P}(3)$

$C(32)-C(33)-C(34)$

$\mathrm{C}(34)-\mathrm{C}(33)-\mathrm{H}(33)$

$\mathrm{C}(35)-\mathrm{C}(34)-\mathrm{H}(34)$

$C(36)-C(35)-C(34)$

$\mathrm{C}(34)-\mathrm{C}(35)-\mathrm{H}(35)$

$\mathrm{C}(35)-\mathrm{C}(36)-\mathrm{H}(36)$

$C(36)-C(37)-C(32)$

$\mathrm{C}(32)-\mathrm{C}(37)-\mathrm{H}(37)$

$C(39)-C(38)-P(3)$

$C(40)-C(39)-C(38)$

$\mathrm{C}(38)-\mathrm{C}(39)-\mathrm{H}(39)$

$\mathrm{C}(41)-\mathrm{C}(40)-\mathrm{H}(40)$

$C(42)-C(41)-C(40)$

$\mathrm{C}(40)-\mathrm{C}(41)-\mathrm{H}(41)$

$\mathrm{C}(41)-\mathrm{C}(42)-\mathrm{H}(42)$

$C(42)-C(43)-C(38)$

$\mathrm{C}(38)-\mathrm{C}(43)-\mathrm{H}(43)$

$C(45)-C(44)-P(4)$

$C(46)-C(45)-C(44)$

$\mathrm{C}(44)-\mathrm{C}(45)-\mathrm{H}(45)$

$\mathrm{C}(47)-\mathrm{C}(46)-\mathrm{H}(46)$

$C(48)-C(47)-C(46)$

$\mathrm{C}(46)-\mathrm{C}(47)-\mathrm{H}(47)$

$\mathrm{C}(47)-\mathrm{C}(48)-\mathrm{H}(48)$

$C(48)-C(49)-C(44)$

$\mathrm{C}(44)-\mathrm{C}(49)-\mathrm{H}(49)$

$\mathrm{C}(51)-\mathrm{C}(50)-\mathrm{P}(4)$

$C(52)-C(51)-C(50)$

$\mathrm{C}(50)-\mathrm{C}(51)-\mathrm{H}(51)$

$\mathrm{C}(53)-\mathrm{C}(52)-\mathrm{H}(52)$

$C(52)-C(53)-C(54)$

C ( 54$)-\mathrm{C}(53)-\mathrm{H}(53)$

$\mathrm{C}(55)-\mathrm{C}(54)-\mathrm{H}(54)$

$C(54)-C(55)-C(50)$

$\mathrm{C}(50)-\mathrm{C}(55)-\mathrm{H}(55)$

$C(57)-C(56)-P(4)$

$C(56)-C(57)-C(58)$

$\mathrm{C}(58)-\mathrm{C}(57)-\mathrm{H}(57)$

$\mathrm{C}(59)-\mathrm{C}(58)-\mathrm{H}(58)$

$C(58)-C(59)-C(60)$

$\mathrm{C}(60)-\mathrm{C}(59)-\mathrm{H}(59)$

$\mathrm{C}(59)-\mathrm{C}(60)-\mathrm{H}(60)$

$C(60)-C(61)-C(56)$

$\mathrm{C}(56)-\mathrm{C}(61)-\mathrm{H}(61)$
$119.4(2)$

120.3

120.2

$121.4(2)$

119.3

$117.7(1)$

$121.0(2)$

119.5

119.8

$119.0(2)$

120.5

119.5

$120.7(2)$

119.7

$121.2(1)$

$121.1(2)$

119.5

120.0

$119.4(2)$

120.3

119.5

$120.0(2)$

120.0

$117.9(2)$

$120.9(2)$

119.5

120.0

$120.3(2)$

119.8

119.9

$120.4(2)$

119.8

$120.5(2)$

$121.1(2)$

119.5

120.0

$120.1(2)$

120.0

120.1

120.9 (2)

119.5

$124.9(2)$

$120.8(2)$

119.6

119.5

$119.3(2)$

120.4

119.9

$121.7(2)$

119.2 
Table S1-4: Anisotropic displacement parameters for 2.

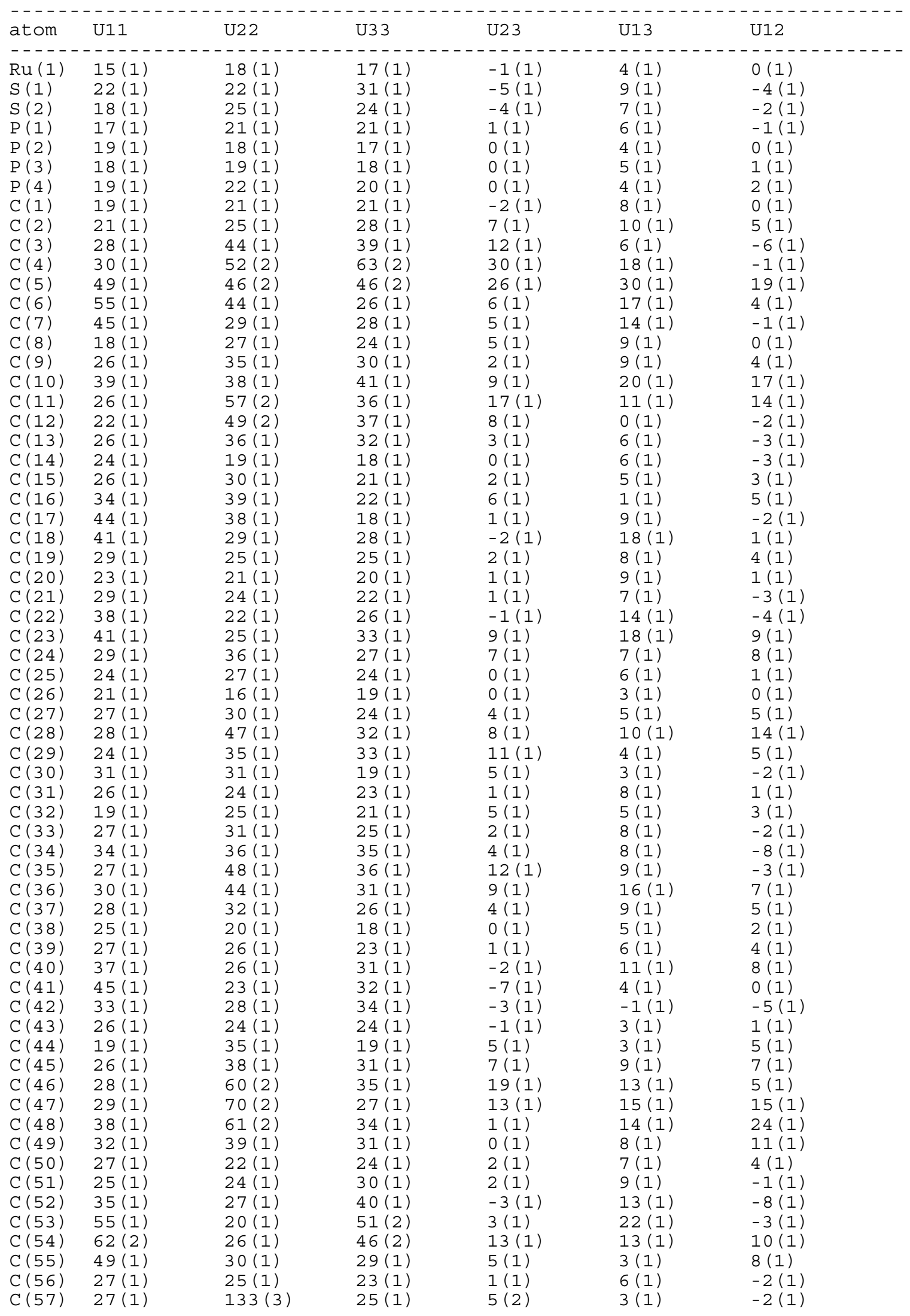




$\begin{array}{lllllll}C(58) & 32(1) & 181(4) & 28(1) & 10(2) & -4(1) & -16(2) \\ C(59) & 60(2) & 63(2) & 20(1) & 0(1) & 3(1) & -23(1) \\ C(60) & 57(1) & 29(1) & 28(1) & 4(1) & 19(1) & 4(1) \\ C(61) & 36(1) & 28(1) & 27(1) & 8(1) & 9(1) & 3(1)\end{array}$

The anisotropic displacement factor exponent takes the form $2 \mathrm{pi}^{\wedge} 2\left[\mathrm{~h}^{\wedge} 2 \mathrm{a}{ }^{\wedge} 2 \mathrm{U}(11)+\ldots+2 \mathrm{hka} * \mathrm{~b} * \mathrm{U}(12)\right]$ 
Table S1-5. Hydrogen Coordinates $\left(A \times 10^{\wedge} 4\right)$ and equivalent isotropic displacement parameters $\left(A^{\wedge} 2 \times 10^{\wedge} 3\right)$ for 2

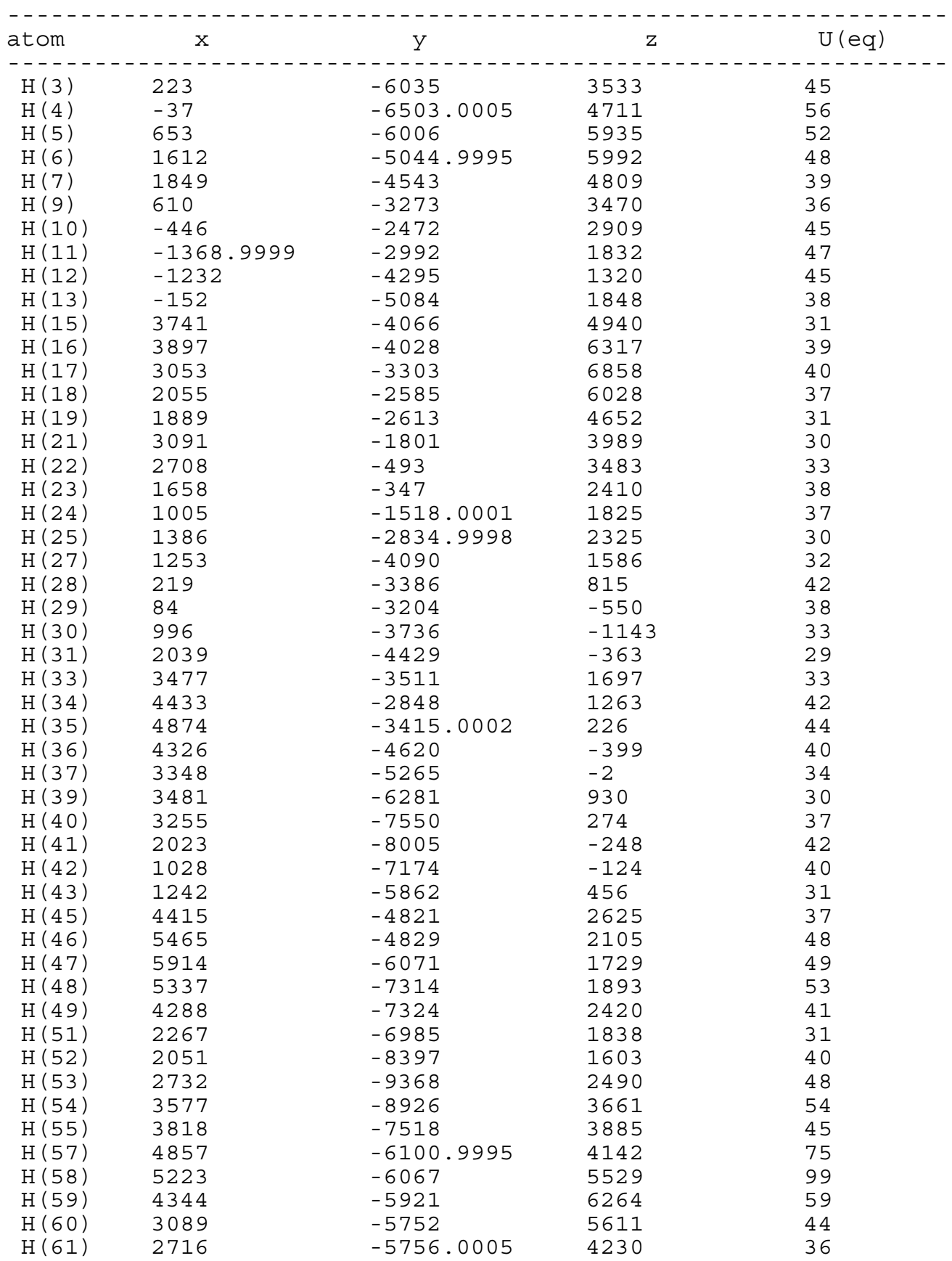


Figure S2: Molecular structure of 3 (S,S)

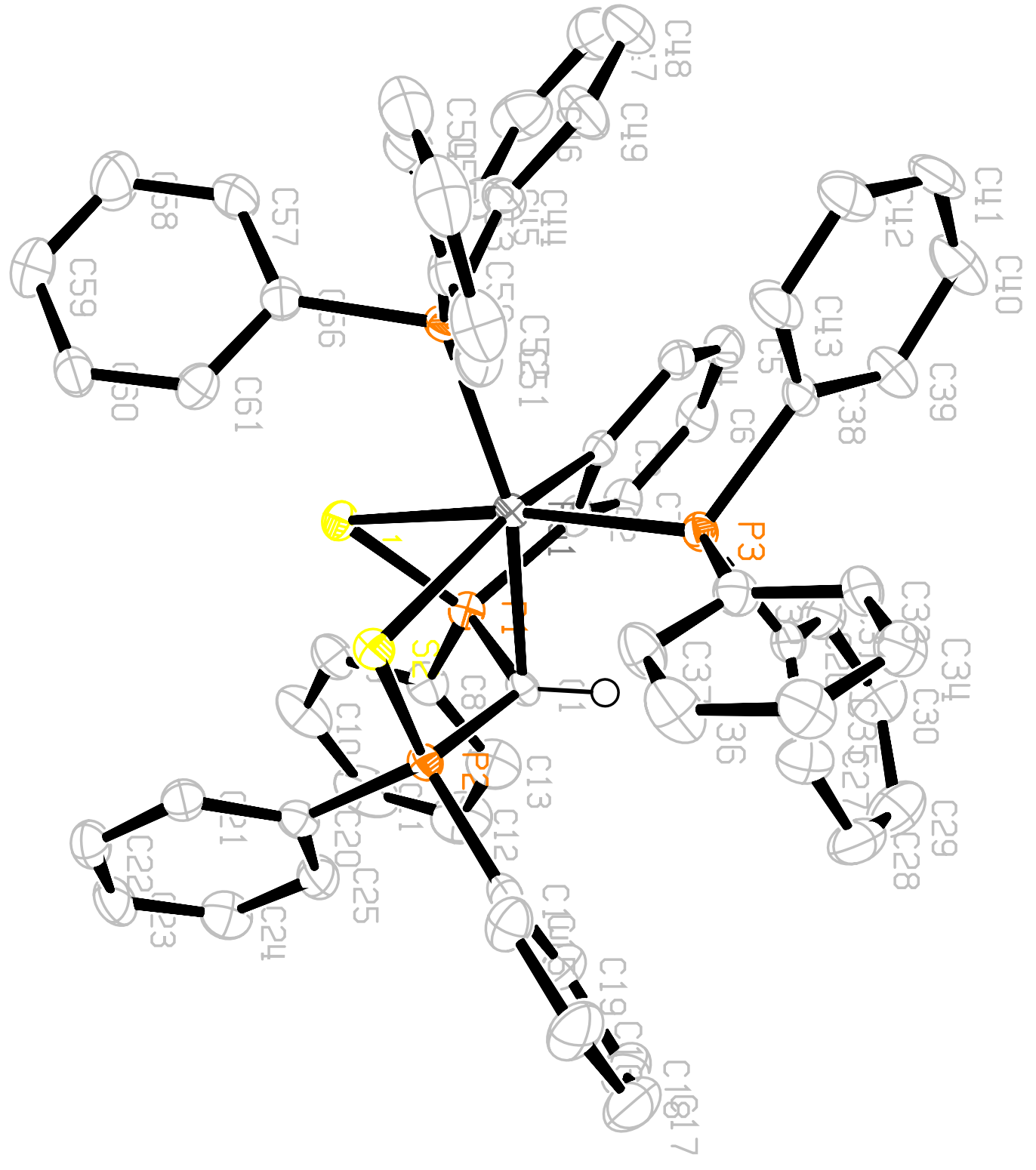




\section{Table S2-1: Crystal data and structure refinement for 3}

\begin{tabular}{|c|c|}
\hline Compound & rucarbormet \\
\hline Molecular formula & $\mathrm{C}_{61} \mathrm{H}_{50} \mathrm{P}_{4} \mathrm{RuS}_{2}, \mathrm{C}_{2} \mathrm{H}_{4} \mathrm{Cl}_{2}$ \\
\hline Molecular weight & 1171.03 \\
\hline Crystal habit & yellow block \\
\hline Crystal dimensions(mm) & $0.24 \times 0.20 \times 0.16$ \\
\hline Crystal system & monoclinic \\
\hline Space group & $\mathrm{P} 2{ }_{1} / \mathrm{c}$ \\
\hline $\mathrm{a}(\AA)$ & $19.696(2)$ \\
\hline $\mathrm{b}(\AA)$ & $29.112(5)$ \\
\hline$c(\AA)$ & $19.044(4)$ \\
\hline$\alpha\left(^{\circ}\right)$ & 90.00 \\
\hline$\beta\left(^{\circ}\right)$ & $91.635(11)$ \\
\hline$\gamma\left({ }^{\circ}\right)$ & 90.00 \\
\hline $\mathrm{V}\left(\AA^{3}\right)$ & $10915(3)$ \\
\hline $\mathrm{Z}$ & 8 \\
\hline $\mathrm{d}\left(\mathrm{g}-\mathrm{cm}^{-3}\right)$ & 1.425 \\
\hline $\mathrm{F}(000)$ & 4816 \\
\hline$\mu\left(\mathrm{cm}^{-1}\right)$ & 0.620 \\
\hline Absorption corrections & multi-scan ; $0.8655 \mathrm{~min}, 0.9073 \max$ \\
\hline Diffractometer & KappaCCD \\
\hline X-ray source & $\operatorname{MoK} \alpha$ \\
\hline$\lambda(\AA)$ & 0.71073 \\
\hline Monochromator & graphite \\
\hline $\mathrm{T}(\mathrm{K})$ & $150.0(1)$ \\
\hline Scan mode & phi and omega scans \\
\hline Maximum $\theta$ & 27.48 \\
\hline HKL ranges & $-250 ;-370 ;-2424$ \\
\hline Reflections measured & 23542 \\
\hline Unique data & 23550 \\
\hline Rint & 0.0320 \\
\hline Reflections used & 20810 \\
\hline Criterion & $\mathrm{I}>2 \sigma \mathrm{I})$ \\
\hline Refinement type & Fsqd \\
\hline Hydrogen atoms & mixed \\
\hline Parameters refined & 1298 \\
\hline Reflections / parameter & 16 \\
\hline wR2 & 0.1487 \\
\hline $\mathrm{R} 1$ & 0.0499 \\
\hline Weights $a, b$ & $0.0585 ; 37.514$ \\
\hline GoF & 1.146 \\
\hline difference peak / hole $\left(\mathrm{e} \AA^{-3}\right)$ & $1.002(0.122) /-0.941(0.122)$ \\
\hline
\end{tabular}


Table S2-2. Atomic Coordinates (A $x$ 10^4) and equivalent isotropic displacement parameters $\left(A^{\wedge} 2 \times 10^{\wedge} 3\right)$ for 3

\begin{tabular}{|c|c|c|c|c|}
\hline atom & $\mathrm{x}$ & Y & z & $\mathrm{U}(\mathrm{eq})$ \\
\hline $\mathrm{Ru}(1)$ & $-4395(1)$ & $-824(1)$ & $-2820(1)$ & $15(1)$ \\
\hline$S(1)$ & $-5278(1)$ & $-206(1)$ & $-2783(1)$ & $18(1)$ \\
\hline$S(2)$ & $-4186(1)$ & $-489(1)$ & $-4072(1)$ & $19(1)$ \\
\hline$P(1)$ & $-4528(1)$ & $-35(1)$ & $-2106(1)$ & $18(1)$ \\
\hline$P(2)$ & $-3765(1)$ & $-4(1)$ & $-3466(1)$ & $17(1)$ \\
\hline$P(3)$ & $-3441(1)$ & $-1286(1)$ & $-2659(1)$ & $18(1)$ \\
\hline P (4) & $-5249(1)$ & $-1338(1)$ & $-3125(1)$ & $18(1)$ \\
\hline$C(1)$ & $-3809(2)$ & $-172(2)$ & $-2576(2)$ & $15(1)$ \\
\hline$C(2)$ & $-4512(2)$ & $-449(2)$ & $-1426(3)$ & $21(1)$ \\
\hline$C(3)$ & $-4514(2)$ & $-882(2)$ & $-1749(2)$ & $19(1)$ \\
\hline$C(4)$ & $-4575(2)$ & $-1252(2)$ & $-1281(3)$ & $22(1)$ \\
\hline$C(5)$ & $-4611(3)$ & $-1183(2)$ & $-559(3)$ & $27(1)$ \\
\hline$C(6)$ & $-4588(3)$ & $-749(2)$ & $-274(3)$ & $28(1)$ \\
\hline$C(7)$ & $-4544(2)$ & $-370(2)$ & $-707(3)$ & $24(1)$ \\
\hline$C(8)$ & $-4605(3)$ & $533(2)$ & $-1729(3)$ & $24(1)$ \\
\hline$C(9)$ & $-5217(3)$ & $763(2)$ & $-1781(3)$ & $29(1)$ \\
\hline C (10) & $-5275(4)$ & $1203(2)$ & $-1518(3)$ & $38(1)$ \\
\hline C (11) & $-4719(4)$ & $1412(2)$ & $-1208(3)$ & $46(2)$ \\
\hline$C(12)$ & $-4107(4)$ & $1179(2)$ & $-1131(4)$ & $46(2)$ \\
\hline$C(13)$ & $-4053(3)$ & $737(2)$ & $-1393(3)$ & $37(1)$ \\
\hline$C(14)$ & $-2876(2)$ & $75(2)$ & $-3638(3)$ & $21(1)$ \\
\hline C (15) & $-2594(3)$ & $-126(2)$ & $-4224(3)$ & $29(1)$ \\
\hline$C(16)$ & $-1913(3)$ & $-67(2)$ & $-4348(3)$ & $39(1)$ \\
\hline C (17) & $-1512(3)$ & $198(2)$ & $-3909(4)$ & $42(2)$ \\
\hline$C(18)$ & $-1781(3)$ & $397(2)$ & $-3322(3)$ & $35(1)$ \\
\hline C (19) & $-2465(3)$ & $339(2)$ & $-3187(3)$ & $28(1)$ \\
\hline$C(20)$ & $-4160(2)$ & $550(2)$ & $-3630(2)$ & $20(1)$ \\
\hline$C(21)$ & $-4625(2)$ & $611(2)$ & $-4180(3)$ & $23(1)$ \\
\hline$C(22)$ & $-4919(3)$ & $1038(2)$ & $-4301(3)$ & $27(1)$ \\
\hline$C(23)$ & $-4771(3)$ & $1397(2)$ & $-3856(3)$ & $33(1)$ \\
\hline C ( 24$)$ & $-4329(3)$ & $1337(2)$ & $-3285(3)$ & $32(1)$ \\
\hline$C(25)$ & $-4015(3)$ & $914(2)$ & $-3173(3)$ & $28(1)$ \\
\hline$C(26)$ & $-2795(2)$ & $-988(2)$ & $-2098(3)$ & $21(1)$ \\
\hline C (27) & $-2232(3)$ & $-785(2)$ & $-2406(3)$ & $32(1)$ \\
\hline$C(28)$ & $-1792(3)$ & $-522(2)$ & $-2005(4)$ & $39(1)$ \\
\hline C (29) & $-1863(3)$ & $-467(2)$ & $-1302(4)$ & $44(2)$ \\
\hline C (30) & $-2422(3)$ & $-660(2)$ & $-993(3)$ & $38(1)$ \\
\hline C (31) & $-2892(3)$ & $-904(2)$ & $-1390(3)$ & $28(1)$ \\
\hline$C(32)$ & $-2873(2)$ & $-1478(2)$ & $-3360(3)$ & $23(1)$ \\
\hline C (33) & $-2357(3)$ & $-1786(2)$ & $-3198(3)$ & $25(1)$ \\
\hline C (34) & $-1890(3)$ & $-1913(2)$ & $-3696(3)$ & $31(1)$ \\
\hline$C(35)$ & $-1945(3)$ & $-1737(2)$ & $-4364(3)$ & $33(1)$ \\
\hline$C(36)$ & $-2454(3)$ & $-1433(2)$ & $-4536(3)$ & $37(1)$ \\
\hline C (37) & $-2920(3)$ & $-1302(2)$ & $-4038(3)$ & $29(1)$ \\
\hline C (38) & $-3543(2)$ & $-1856(2)$ & $-2232(3)$ & $20(1)$ \\
\hline C (39) & $-3370(3)$ & $-1960(2)$ & $-1551(3)$ & $31(1)$ \\
\hline$C(40)$ & $-3496(3)$ & $-2395(2)$ & $-1280(3)$ & $40(2)$ \\
\hline C (41) & $-3778(3)$ & $-2728(2)$ & $-1691(4)$ & $40(2)$ \\
\hline C (42) & $-3931(3)$ & $-2636(2)$ & $-2384(4)$ & $41(1)$ \\
\hline C (43) & $-3827(3)$ & $-2201(2)$ & $-2651(3)$ & $36(1)$ \\
\hline$C(44)$ & $-5628(3)$ & $-1649(2)$ & $-2411(3)$ & $26(1)$ \\
\hline$C(45)$ & $-5973(3)$ & $-1390(2)$ & $-1919(3)$ & $33(1)$ \\
\hline$C(46)$ & $-6255(3)$ & $-1592(2)$ & $-1334(3)$ & $42(2)$ \\
\hline C (47) & $-6172(4)$ & $-2052(3)$ & $-1221(3)$ & $47(2)$ \\
\hline$C(48)$ & $-5824(4)$ & $-2312(2)$ & $-1682(4)$ & $44(2)$ \\
\hline C (49) & $-5546(3)$ & $-2116(2)$ & $-2284(3)$ & $37(1)$ \\
\hline$C(50)$ & $-5127(3)$ & $-1775(2)$ & $-3801(3)$ & $23(1)$ \\
\hline C (51) & $-4546(3)$ & $-1759(2)$ & $-4189(3)$ & $28(1)$ \\
\hline C ( 52$)$ & $-4440(3)$ & $-2066(2)$ & $-4727(3)$ & $36(1)$ \\
\hline C (53) & $-4921(4)$ & $-2398(2)$ & $-4894(3)$ & $43(2)$ \\
\hline$C(54)$ & $-5513(4)$ & $-2412(2)$ & $-4523(3)$ & $40(2)$ \\
\hline$C(55)$ & $-5621(3)$ & $-2104(2)$ & $-3981(3)$ & $32(1)$ \\
\hline C ( 56$)$ & $-6018(2)$ & $-1072(2)$ & $-3527(3)$ & $22(1)$ \\
\hline
\end{tabular}




\begin{tabular}{|c|c|c|c|c|}
\hline$C(57)$ & $-6675(3)$ & -1181 ( 2) & $-3353(3)$ & $34(1)$ \\
\hline$C(58)$ & $-7237(3)$ & $-983(2)$ & $-3685(4)$ & $44(2)$ \\
\hline$C(59)$ & $-7146(3)$ & $-668(2)$ & $-4222(3)$ & $35(1)$ \\
\hline$C(60)$ & $-6494(3)$ & $-552(2)$ & $-4409(3)$ & $28(1)$ \\
\hline$C(61)$ & $-5941(3)$ & $-754(2)$ & $-4069(3)$ & $24(1)$ \\
\hline $\mathrm{Ru}(2)$ & $-760(1)$ & $-4111(1)$ & $-2728(1)$ & $16(1$ \\
\hline$S(3)$ & $195(1)$ & $-4685(1)$ & $-2726(1)$ & $21(1$ \\
\hline$S(4)$ & $-1019(1)$ & $-4362(1)$ & $-4003(1)$ & $21(1$ \\
\hline$P(5)$ & $-517(1)$ & $-4929(1)$ & $-2110(1)$ & $20(1$ \\
\hline$P(6)$ & $-1327(1)$ & $-4918(1)$ & $-3493(1)$ & $18(1)$ \\
\hline $\mathrm{P}(7)$ & $-1732(1)$ & $-3683(1)$ & $-2568(1)$ & $18(1)$ \\
\hline $\mathrm{P}(8)$ & $42(1)$ & $-3560(1)$ & $-3017(1)$ & $20(1)$ \\
\hline$C(62)$ & $-1262(2)$ & $-4793(2)$ & $-2585(3)$ & $21(1)$ \\
\hline$C(63)$ & $-535(2)$ & $-4554(2)$ & $-1378(2)$ & $20(1)$ \\
\hline$C(64)$ & $-598(2)$ & $-4106(2)$ & $-1646(2)$ & $18(1$ \\
\hline$C(65)$ & $-594(2)$ & $-3753(2)$ & $-1139(3)$ & $23(1$ \\
\hline$C(66)$ & $-528(3)$ & $-3851(2)$ & $-429(3)$ & $27(1$ \\
\hline$C(67)$ & $-470(3)$ & $-4302(2)$ & $-184(3)$ & $30(1$ \\
\hline$C(68)$ & $-470(2)$ & $-4657(2)$ & $-661(3)$ & $25(1$ \\
\hline$C(69)$ & $-396(3)$ & $-5520(2)$ & $-1831(3)$ & $28(1$ \\
\hline$C(70)$ & $162(4)$ & $-5757(2)$ & $-2020(5)$ & $67(3$ \\
\hline$C(71)$ & $259(5)$ & $-6206(3)$ & $-1808(5)$ & $79(3$ \\
\hline$C(72)$ & $-199(4)$ & $-6418(2)$ & $-1402(4)$ & $60(2$ \\
\hline$C(73)$ & $-760(4)$ & $-6178(2)$ & $-1193(4)$ & $58(2$ \\
\hline$C(74)$ & $-867(3)$ & $-5725(2)$ & $-1399(4)$ & $42(2$ \\
\hline$C(75)$ & $-891(3)$ & $-5433(2)$ & $-3758(3)$ & $25(1$ \\
\hline$C(76)$ & $-284(3)$ & $-5413(2)$ & $-4103(3)$ & $28(1$ \\
\hline$C(77)$ & $47(3)$ & $-5813(2)$ & $-4303(3)$ & $39(1$ \\
\hline$C(78)$ & $-253(4)$ & $-6229(2)$ & $-4184(4)$ & $55(2$ \\
\hline$C(79)$ & $-876(4)$ & $-6256(2)$ & $-3864(5)$ & $61(2$ \\
\hline$C(80)$ & $-1180(3)$ & $-5859(2)$ & $-3643(4)$ & $45(2$ \\
\hline$C(81)$ & $-2202(2)$ & $-5049(2)$ & $-3719(3)$ & $22(1$ \\
\hline$C(82)$ & $-2690(3)$ & $-5134(2)$ & $-3224(3)$ & $33(1$ \\
\hline$C(83)$ & $-3361(3)$ & $-5224(2)$ & $-3449(4)$ & $40(1$ \\
\hline$C(84)$ & $-3534(3)$ & $-5235(2)$ & $-4137(4)$ & $43(2$ \\
\hline$C(85)$ & $-3052(3)$ & $-5147(2)$ & $-4629(3)$ & $38(1$ \\
\hline$C(86)$ & $-2394(3)$ & $-5055(2)$ & $-4423(3)$ & $28(1$ \\
\hline$C(87)$ & $-2268(2)$ & $-3501(2)$ & $-3328(2)$ & $18(1$ \\
\hline$C(88)$ & $-2541(3)$ & $-3842(2)$ & $-3770(3)$ & $28(1$ \\
\hline$C(89)$ & $-2970(3)$ & $-3736(2)$ & $-4320(3)$ & $34(1$ \\
\hline$C(90)$ & $-3150(3)$ & $-3283(2)$ & $-4452(3)$ & $34(1$ \\
\hline$C(91)$ & $-2892(3)$ & $-2940(2)$ & $-4026(3)$ & $39(1$ \\
\hline$C(92)$ & $-2460(3)$ & $-3052(2)$ & $-3461(3)$ & $29(1$ \\
\hline$C(93)$ & $-1649(2)$ & $-3135(2)$ & $-2089(3)$ & $22(1$ \\
\hline$C(94)$ & $-1961(3)$ & $-3050(2)$ & $-1455(3)$ & $30(1$ \\
\hline$C(95)$ & $-1877(3)$ & $-2633(2)$ & $-1122(3)$ & $38(1$ \\
\hline$C(96)$ & $-1501(3)$ & $-2285(2)$ & $-1402(3)$ & $38(1$ \\
\hline C (97) & $-1186(3)$ & $-2365(2)$ & $-2029(3)$ & $33(1$ \\
\hline$C(98)$ & $-1253(3)$ & $-2792(2)$ & $-2364(3)$ & $25(1$ \\
\hline C (99) & $-2406(2)$ & $-3960(2)$ & $-2054(2)$ & $20(1$ \\
\hline$C\left(\begin{array}{lll}1 & 0 & 0\end{array}\right)$ & $-2235(2)$ & $-4245(2)$ & $-1490(3)$ & $23(1$ \\
\hline$C(101)$ & $-2732(3)$ & $-4446(2)$ & $-1093(3)$ & $29(1$ \\
\hline$C(102)$ & $-3408(3)$ & $-4359(2)$ & $-1240(3)$ & $31(1$ \\
\hline$C(103)$ & $-3585(3)$ & $-4066(2)$ & $-1781(3)$ & $32(1$ \\
\hline$C(104)$ & $-3083(3)$ & $-3867(2)$ & $-2185(3)$ & $28(1$ \\
\hline$C(105)$ & $-132(3)$ & $-3164(2)$ & $-3752(3)$ & $25(1$ \\
\hline$C(106)$ & $-757(3)$ & $-3158(2)$ & $-4086(3)$ & 29( \\
\hline$C(107)$ & $-897(3)$ & $-2865(2)$ & $-4649(3)$ & $36(1)$ \\
\hline$C(108)$ & $-398(4)$ & $-2577(2)$ & $-4888(3)$ & $40(1$ \\
\hline$C(109)$ & $233(3)$ & $-2587(2)$ & $-4566(3)$ & $40(1$ \\
\hline$C(110)$ & $375(3)$ & $-2877(2)$ & $-4010(3)$ & $36(1$ \\
\hline$C(111)$ & $405(3)$ & $-3180(2)$ & $-2330(3)$ & $31(1$ \\
\hline$C(112)$ & $381(3)$ & $-2705(2)$ & $-2363(4)$ & $43(2$ \\
\hline$C(113)$ & $629(4)$ & $-2430(3)$ & $-1840(5)$ & $63(2$ \\
\hline$C(114)$ & $927(4)$ & $-2626(3)$ & $-1275(5)$ & $65(3) x$ \\
\hline$C(115)$ & $962(4)$ & $-3102(3)$ & $-1205(4)$ & $58(2$ \\
\hline$C(116)$ & $691(3)$ & $-3384(3)$ & $-1745(3)$ & 44( \\
\hline C (117) & $825(2)$ & $-3811(2)$ & $-3365(3)$ & $23(1$ \\
\hline$C(118)$ & $1472(2)$ & $-3733(2)$ & $-3081(3)$ & $26(1$ \\
\hline C (119) & $2037(3)$ & $-3916(2)$ & $-3402(3)$ & $35(1$ \\
\hline
\end{tabular}




$\begin{array}{lllll}C(120) & 1963(3) & -4170(2) & -4009(3) & 38(1) \\ C(121) & 1323(3) & -4247(2) & -4298(3) & 36(1) \\ C(122) & 756(3) & -4068(2) & -3979(3) & 30(1) \\ C 1(1) & 2649(1) & -3369(1) & -1567(1) & 54(1) \\ C 1(2) & -7386(1) & -2834(1) & -16(1) & 72(1) \\ C(123) & 2791(4) & -3626(2) & -726(4) & 46(2) \\ C(124) & -6857(4) & -1696(2) & -5230(4) & 52(2) \\ C 1(3) & -6930(2) & -2895(1) & -3214(2) & 103(1) \\ C 1(4) & -1910(2) & -6761(1) & -2513(3) & 152(2) \\ C(125) & -7580(10) & -2444(6) & -3153(8) & 143(6) \\ C(126) & -7573(7) & -2266(4) & -2504(7) & 108(4)\end{array}$

$\mathrm{U}(\mathrm{eq})$ is defined as $1 / 3$ the trace of the Uij tensor. 
Table S2-3. Bond lengths (A) and angles (deg) for 3

\begin{tabular}{|c|c|c|c|}
\hline $\mathrm{Ru}(1)-\mathrm{C}(3)$ & $2.066(5)$ & $\operatorname{Ru}(1)-C(1)$ & $2.262(4)$ \\
\hline $\mathrm{Ru}(1)-\mathrm{P}(4)$ & $2.314(1)$ & $R u(1)-P(3)$ & $2.322(1)$ \\
\hline $\mathrm{Ru}(1)-\mathrm{S}(1)$ & $2.504(1)$ & $R u(1)-S(2)$ & $2.620(1)$ \\
\hline $\mathrm{Ru}(1)-\mathrm{P}(1)$ & $2.687(1)$ & $S(1)-P(1)$ & $1.996(2)$ \\
\hline$S(2)-P(2)$ & $1.990(2)$ & $P(1)-C(1)$ & $1.745(4)$ \\
\hline$P(1)-C(2)$ & $1.769(5)$ & $P(1)-C(8)$ & $1.809(5)$ \\
\hline$P(2)-C(1)$ & $1.768(4)$ & $P(2)-C(14)$ & $1.805(5)$ \\
\hline$P(2)-C(20)$ & $1.815(5)$ & $P(3)-C(26)$ & $1.853(5)$ \\
\hline$P(3)-C(32)$ & $1.854(5)$ & $P(3)-C(38)$ & $1.861(5)$ \\
\hline$P(4)-C(44)$ & $1.813(5)$ & $P(4)-C(50)$ & $1.829(5)$ \\
\hline$P(4)-C(56)$ & $1.848(5)$ & $\mathrm{C}(1)-\mathrm{H}(1)$ & 0.9500 \\
\hline$C(2)-C(7)$ & $1.392(7)$ & $C(2)-C(3)$ & $1.404(7)$ \\
\hline$C(3)-C(4)$ & $1.405(7)$ & $C(4)-C(5)$ & $1.393(7)$ \\
\hline $\mathrm{C}(4)-\mathrm{H}(4)$ & 0.9500 & $C(5)-C(6)$ & $1.377(8)$ \\
\hline $\mathrm{C}(5)-\mathrm{H}(5)$ & 0.9500 & $C(6)-C(7)$ & $1.383(7)$ \\
\hline $\mathrm{C}(6)-\mathrm{H}(6)$ & 0.9500 & $\mathrm{C}(7)-\mathrm{H}(7)$ & 0.9500 \\
\hline$C(8)-C(9)$ & $1.378(7)$ & $C(8)-C(13)$ & $1.381(8)$ \\
\hline$C(9)-C(10)$ & $1.382(7)$ & $\mathrm{C}(9)-\mathrm{H}(9)$ & 0.9500 \\
\hline$C(10)-C(11)$ & $1.37(1)$ & $\mathrm{C}(10)-\mathrm{H}(10)$ & 0.9500 \\
\hline$C(11)-C(12)$ & $1.39(1)$ & $\mathrm{C}(11)-\mathrm{H}(11)$ & 0.9500 \\
\hline$C(12)-C(13)$ & $1.386(8)$ & $\mathrm{C}(12)-\mathrm{H}(12)$ & 0.9500 \\
\hline $\mathrm{C}(13)-\mathrm{H}(13)$ & 0.9500 & $C(14)-C(15)$ & $1.390(7)$ \\
\hline$C(14)-C(19)$ & $1.393(7)$ & $C(15)-C(16)$ & $1.381(7)$ \\
\hline $\mathrm{C}(15)-\mathrm{H}(15)$ & 0.9500 & $C(16)-C(17)$ & $1.370(9)$ \\
\hline $\mathrm{C}(16)-\mathrm{H}(16)$ & 0.9500 & $C(17)-C(18)$ & $1.378(9)$ \\
\hline $\mathrm{C}(17)-\mathrm{H}(17)$ & 0.9500 & $C(18)-C(19)$ & $1.390(8)$ \\
\hline $\mathrm{C}(18)-\mathrm{H}(18)$ & 0.9500 & $\mathrm{C}(19)-\mathrm{H}(19)$ & 0.9500 \\
\hline$C(20)-C(21)$ & $1.384(7)$ & $C(20)-C(25)$ & $1.394(7)$ \\
\hline$C(21)-C(22)$ & $1.389(7)$ & $\mathrm{C}(21)-\mathrm{H}(21)$ & 0.9500 \\
\hline$C(22)-C(23)$ & $1.371(8)$ & $\mathrm{C}(22)-\mathrm{H}(22)$ & 0.9500 \\
\hline$C(23)-C(24)$ & $1.384(8)$ & $\mathrm{C}(23)-\mathrm{H}(23)$ & 0.9500 \\
\hline$C(24)-C(25)$ & $1.391(7)$ & $\mathrm{C}(24)-\mathrm{H}(24)$ & 0.9500 \\
\hline $\mathrm{C}(25)-\mathrm{H}(25)$ & 0.9500 & $C(26)-C(31)$ & $1.389(7)$ \\
\hline$C(26)-C(27)$ & $1.401(7)$ & $C(27)-C(28)$ & $1.373(8)$ \\
\hline $\mathrm{C}(27)-\mathrm{H}(27)$ & 0.9500 & $C(28)-C(29)$ & $1.360(9)$ \\
\hline $\mathrm{C}(28)-\mathrm{H}(28)$ & 0.9500 & $C(29)-C(30)$ & $1.383(9)$ \\
\hline $\mathrm{C}(29)-\mathrm{H}(29)$ & 0.9500 & $C(30)-C(31)$ & $1.375(8)$ \\
\hline $\mathrm{C}(30)-\mathrm{H}(30)$ & 0.9500 & $\mathrm{C}(31)-\mathrm{H}(31)$ & 0.9500 \\
\hline$C(32)-C(33)$ & $1.384(7)$ & $C(32)-C(37)$ & $1.389(7)$ \\
\hline$C(33)-C(34)$ & $1.390(7)$ & $\mathrm{C}(33)-\mathrm{H}(33)$ & 0.9500 \\
\hline$C(34)-C(35)$ & $1.373(8)$ & $\mathrm{C}(34)-\mathrm{H}(34)$ & 0.9500 \\
\hline$C(35)-C(36)$ & $1.369(8)$ & $\mathrm{C}(35)-\mathrm{H}(35)$ & 0.9500 \\
\hline$C(36)-C(37)$ & $1.392(7)$ & $\mathrm{C}(36)-\mathrm{H}(36)$ & 0.9500 \\
\hline $\mathrm{C}(37)-\mathrm{H}(37)$ & 0.9500 & $C(38)-C(39)$ & $1.366(7)$ \\
\hline$C(38)-C(43)$ & $1.391(7)$ & $C(39)-C(40)$ & $1.393(8)$ \\
\hline $\mathrm{C}(39)-\mathrm{H}(39)$ & 0.9500 & $C(40)-C(41)$ & $1.35(1)$ \\
\hline $\mathrm{C}(40)-\mathrm{H}(40)$ & 0.9500 & $C(41)-C(42)$ & $1.372(9)$ \\
\hline $\mathrm{C}(41)-\mathrm{H}(41)$ & 0.9500 & $C(42)-C(43)$ & $1.382(8)$ \\
\hline $\mathrm{C}(42)-\mathrm{H}(42)$ & 0.9500 & $\mathrm{C}(43)-\mathrm{H}(43)$ & 0.9500 \\
\hline$C(44)-C(49)$ & $1.390(7)$ & $C(44)-C(45)$ & $1.394(8)$ \\
\hline$C(45)-C(46)$ & $1.389(8)$ & $\mathrm{C}(45)-\mathrm{H}(45)$ & 0.9500 \\
\hline$C(46)-C(47)$ & $1.37(1)$ & $\mathrm{C}(46)-\mathrm{H}(46)$ & 0.9500 \\
\hline$C(47)-C(48)$ & $1.36(1)$ & $\mathrm{C}(47)-\mathrm{H}(47)$ & 0.9500 \\
\hline$C(48)-C(49)$ & $1.405(9)$ & $\mathrm{C}(48)-\mathrm{H}(48)$ & 0.9500 \\
\hline $\mathrm{C}(49)-\mathrm{H}(49)$ & 0.9500 & $C(50)-C(51)$ & $1.381(7)$ \\
\hline$C(50)-C(55)$ & $1.401(7)$ & $C(51)-C(52)$ & $1.380(7)$ \\
\hline $\mathrm{C}(51)-\mathrm{H}(51)$ & 0.9500 & $C(52)-C(53)$ & $1.384(9)$ \\
\hline $\mathrm{C}(52)-\mathrm{H}(52)$ & 0.9500 & $C(53)-C(54)$ & $1.38(1)$ \\
\hline $\mathrm{C}(53)-\mathrm{H}(53)$ & 0.9500 & $C(54)-C(55)$ & $1.388(8)$ \\
\hline $\mathrm{C}(54)-\mathrm{H}(54)$ & 0.9500 & $\mathrm{C}(55)-\mathrm{H}(55)$ & 0.9500 \\
\hline$C(56)-C(57)$ & $1.383(7)$ & $C(56)-C(61)$ & $1.396(7)$ \\
\hline$C(57)-C(58)$ & $1.385(8)$ & $\mathrm{C}(57)-\mathrm{H}(57)$ & 0.9500 \\
\hline$C(58)-C(59)$ & $1.390(9)$ & $C(58)-H(58)$ & 0.9500 \\
\hline$C(59)-C(60)$ & $1.384(8)$ & $\mathrm{C}(59)-\mathrm{H}(59)$ & 0.9500 \\
\hline$C(60)-C(61)$ & $1.382(7)$ & $\mathrm{C}(60)-\mathrm{H}(60)$ & 0.9500 \\
\hline $\mathrm{C}(61)-\mathrm{H}(61)$ & 0.9500 & $R u(2)-C(64)$ & $2.075(4)$ \\
\hline$R u(2)-C(62)$ & $2.239(5)$ & $R u(2)-P(7)$ & $2.311(1)$ \\
\hline
\end{tabular}




\begin{tabular}{|c|c|c|c|}
\hline $\mathrm{Ru}(2)-\mathrm{P}(8)$ & $2.328(1)$ & $\mathrm{Ru}(2)-\mathrm{S}(3)$ & $2.516(1)$ \\
\hline$R u(2)-S(4)$ & $2.574(1)$ & $\mathrm{Ru}(2)-\mathrm{P}(5)$ & $2.694(1)$ \\
\hline$S(3)-P(5)$ & $1.987(2)$ & $S(4)-P(6)$ & $1.990(2)$ \\
\hline$P(5)-C(62)$ & $1.748(5)$ & $P(5)-C(63)$ & $1.774(5)$ \\
\hline$P(5)-C(69)$ & $1.814(5)$ & $P(6)-C(62)$ & $1.769(5)$ \\
\hline$P(6)-C(81)$ & $1.805(5)$ & $P(6)-C(75)$ & $1.808(5)$ \\
\hline$P(7)-C(93)$ & $1.843(5)$ & $\mathrm{P}(7)-\mathrm{C}(87)$ & $1.845(5)$ \\
\hline$P(7)-C(99)$ & $1.856(5)$ & $P(8)-C(105)$ & $1.837(5)$ \\
\hline$P(8)-C(111)$ & $1.842(5)$ & $P(8)-C(117)$ & $1.847(5)$ \\
\hline $\mathrm{C}(62)-\mathrm{H}(62)$ & 0.9500 & $C(63)-C(68)$ & $1.400(6)$ \\
\hline$C(63)-C(64)$ & $1.405(7)$ & $C(64)-C(65)$ & $1.411(7)$ \\
\hline$C(65)-C(66)$ & $1.384(7)$ & $\mathrm{C}(65)-\mathrm{H}(65)$ & 0.9500 \\
\hline$C(66)-C(67)$ & $1.396(8)$ & $\mathrm{C}(66)-\mathrm{H}(66)$ & 0.9500 \\
\hline$C(67)-C(68)$ & $1.377(8)$ & $\mathrm{C}(67)-\mathrm{H}(67)$ & 0.9500 \\
\hline $\mathrm{C}(68)-\mathrm{H}(68)$ & 0.9500 & $C(69)-C(70)$ & $1.356(9)$ \\
\hline$C(69)-C(74)$ & $1.391(8)$ & $C(70)-C(71)$ & $1.379(9)$ \\
\hline $\mathrm{C}(70)-\mathrm{H}(70)$ & 0.9500 & $C(71)-C(72)$ & $1.35(1)$ \\
\hline $\mathrm{C}(71)-\mathrm{H}(71)$ & 0.9500 & $C(72)-C(73)$ & $1.38(1)$ \\
\hline $\mathrm{C}(72)-\mathrm{H}(72)$ & 0.9500 & $C(73)-C(74)$ & $1.390(9)$ \\
\hline $\mathrm{C}(73)-\mathrm{H}(73)$ & 0.9500 & $\mathrm{C}(74)-\mathrm{H}(74)$ & 0.9500 \\
\hline$C(75)-C(80)$ & $1.383(8)$ & $C(75)-C(76)$ & $1.383(7)$ \\
\hline$C(76)-C(77)$ & $1.391(8)$ & $\mathrm{C}(76)-\mathrm{H}(76)$ & 0.9500 \\
\hline$C(77)-C(78)$ & $1.37(1)$ & $\mathrm{C}(77)-\mathrm{H}(77)$ & 0.9500 \\
\hline$C(78)-C(79)$ & $1.39(1)$ & $\mathrm{C}(78)-\mathrm{H}(78)$ & 0.9500 \\
\hline$C(79)-C(80)$ & $1.374(9)$ & $\mathrm{C}(79)-\mathrm{H}(79)$ & 0.9500 \\
\hline $\mathrm{C}(80)-\mathrm{H}(80)$ & 0.9500 & $C(81)-C(86)$ & $1.382(7)$ \\
\hline$C(81)-C(82)$ & $1.388(7)$ & $C(82)-C(83)$ & $1.401(8)$ \\
\hline $\mathrm{C}(82)-\mathrm{H}(82)$ & 0.9500 & $C(83)-C(84)$ & $1.344(9)$ \\
\hline $\mathrm{C}(83)-\mathrm{H}(83)$ & 0.9500 & $C(84)-C(85)$ & $1.376(9)$ \\
\hline $\mathrm{C}(84)-\mathrm{H}(84)$ & 0.9500 & $C(85)-C(86)$ & $1.370(7)$ \\
\hline $\mathrm{C}(85)-\mathrm{H}(85)$ & 0.9500 & $\mathrm{C}(86)-\mathrm{H}(86)$ & 0.9500 \\
\hline$C(87)-C(92)$ & $1.382(7)$ & $C(87)-C(88)$ & $1.399(7)$ \\
\hline$C(88)-C(89)$ & $1.363(7)$ & $\mathrm{C}(88)-\mathrm{H}(88)$ & 0.9500 \\
\hline$C(89)-C(90)$ & $1.386(8)$ & $\mathrm{C}(89)-\mathrm{H}(89)$ & 0.9500 \\
\hline$C(90)-C(91)$ & $1.374(9)$ & $\mathrm{C}(90)-\mathrm{H}(90)$ & 0.9500 \\
\hline$C(91)-C(92)$ & $1.392(8)$ & $\mathrm{C}(91)-\mathrm{H}(91)$ & 0.9500 \\
\hline $\mathrm{C}(92)-\mathrm{H}(92)$ & 0.9500 & $C(93)-C(98)$ & $1.380(7)$ \\
\hline$C(93)-C(94)$ & $1.392(7)$ & $C(94)-C(95)$ & $1.377(8)$ \\
\hline $\mathrm{C}(94)-\mathrm{H}(94)$ & 0.9500 & $C(95)-C(96)$ & $1.372(9)$ \\
\hline $\mathrm{C}(95)-\mathrm{H}(95)$ & 0.9500 & $C(96)-C(97)$ & $1.380(9)$ \\
\hline $\mathrm{C}(96)-\mathrm{H}(96)$ & 0.9500 & $C(97)-C(98)$ & $1.401(7)$ \\
\hline $\mathrm{C}(97)-\mathrm{H}(97)$ & 0.9500 & $\mathrm{C}(98)-\mathrm{H}(98)$ & 0.9500 \\
\hline$C(99)-C(104)$ & $1.377(7)$ & $C(99)-C(100)$ & $1.390(7)$ \\
\hline$C(100)-C(101)$ & $1.385(7)$ & $\mathrm{C}(100)-\mathrm{H}(100)$ & 0.9500 \\
\hline$C(101)-C(102)$ & $1.376(8)$ & $\mathrm{C}(101)-\mathrm{H}(101)$ & 0.9500 \\
\hline$C(102)-C(103)$ & $1.374(8)$ & $\mathrm{C}(102)-\mathrm{H}(102)$ & 0.9500 \\
\hline$C(103)-C(104)$ & $1.396(7)$ & $\mathrm{C}(103)-\mathrm{H}(103)$ & 0.9500 \\
\hline $\mathrm{C}(104)-\mathrm{H}(104)$ & 0.9500 & $C(105)-C(106)$ & $1.369(8)$ \\
\hline$C(105)-C(110)$ & $1.402(7)$ & $C(106)-C(107)$ & $1.391(7)$ \\
\hline $\mathrm{C}(106)-\mathrm{H}(106)$ & 0.9500 & $C(107)-C(108)$ & $1.379(9)$ \\
\hline $\mathrm{C}(107)-\mathrm{H}(107)$ & 0.9500 & $C(108)-C(109)$ & $1.37(1)$ \\
\hline $\mathrm{C}(108)-\mathrm{H}(108)$ & 0.9500 & $C(109)-C(110)$ & $1.376(8)$ \\
\hline $\mathrm{C}(109)-\mathrm{H}(109)$ & 0.9500 & $\mathrm{C}(110)-\mathrm{H}(110)$ & 0.9500 \\
\hline$C(111)-C(116)$ & $1.369(9)$ & $C(111)-C(112)$ & $1.385(9)$ \\
\hline$C(112)-C(113)$ & $1.357(9)$ & $\mathrm{C}(112)-\mathrm{H}(112)$ & 0.9500 \\
\hline$C(113)-C(114)$ & $1.34(1)$ & $\mathrm{C}(113)-\mathrm{H}(113)$ & 0.9500 \\
\hline$C(114)-C(115)$ & $1.39(1)$ & $\mathrm{C}(114)-\mathrm{H}(114)$ & 0.9500 \\
\hline$C(115)-C(116)$ & $1.410(9)$ & $\mathrm{C}(115)-\mathrm{H}(115)$ & 0.9500 \\
\hline $\mathrm{C}(116)-\mathrm{H}(116)$ & 0.9500 & $C(117)-C(118)$ & $1.389(7)$ \\
\hline$C(117)-C(122)$ & $1.390(7)$ & $C(118)-C(119)$ & $1.393(8)$ \\
\hline $\mathrm{C}(118)-\mathrm{H}(118)$ & 0.9500 & $C(119)-C(120)$ & $1.377(9)$ \\
\hline $\mathrm{C}(119)-\mathrm{H}(119)$ & 0.9500 & $C(120)-C(121)$ & $1.380(8)$ \\
\hline $\mathrm{C}(120)-\mathrm{H}(120)$ & 0.9500 & $C(121)-C(122)$ & $1.389(8)$ \\
\hline $\mathrm{C}(121)-\mathrm{H}(121)$ & 0.9500 & $\mathrm{C}(122)-\mathrm{H}(122)$ & 0.9500 \\
\hline $\mathrm{Cl}(1)-\mathrm{C}(123)$ & $1.782(7)$ & $\mathrm{Cl}(2)-\mathrm{C}(124) \# 4$ & $1.775(8)$ \\
\hline$C(123)-C(124) \# 4$ & $1.489(9)$ & $\mathrm{C}(123)-\mathrm{H}(12 \mathrm{~A})$ & 0.9900 \\
\hline $\mathrm{C}(123)-\mathrm{H}(12 \mathrm{~B})$ & 0.9900 & $C(124)-C(123) \# 4$ & $1.489(9)$ \\
\hline$C(124)-C 1(2) \# 4$ & $1.775(8)$ & $\mathrm{C}(124)-\mathrm{H}(12 \mathrm{C})$ & 0.9900 \\
\hline $\mathrm{C}(124)-\mathrm{H}(12 \mathrm{D})$ & 0.9900 & $C l(3)-C(125)$ & $1.85(2)$ \\
\hline $\mathrm{Cl}(4)-\mathrm{C}(126) \# 2$ & $1.79(1)$ & $C(125)-C(126)$ & $1.34(2)$ \\
\hline
\end{tabular}




$$
\begin{aligned}
& \mathrm{C}(125)-\mathrm{H}(12 \mathrm{E}) \\
& \mathrm{C}(126)-\mathrm{Cl}(4) \# 2 \\
& \mathrm{C}(126)-\mathrm{H}(12 \mathrm{H})
\end{aligned}
$$

0.9900
$1.79(1)$
0.9900

0.9900
$\mathrm{C}(125)-\mathrm{H}(12 \mathrm{~F})$

$\mathrm{C}(126)-\mathrm{H}(12 \mathrm{G})$
$C(3)-R u(1)-P(4)$

$C(3)-R u(1)-P(3)$

$\mathrm{P}(4)-\mathrm{Ru}(1)-\mathrm{P}(3)$

$\mathrm{C}(1)-\mathrm{Ru}(1)-\mathrm{S}(1)$

$\mathrm{P}(3)-\mathrm{Ru}(1)-\mathrm{S}(1)$

$\mathrm{C}(1)-\mathrm{Ru}(1)-\mathrm{S}(2)$

$\mathrm{P}(3)-\mathrm{Ru}(1)-\mathrm{S}(2)$

$\mathrm{C}(3)-\mathrm{Ru}(1)-\mathrm{P}(1)$

$\mathrm{P}(4)-\mathrm{Ru}(1)-\mathrm{P}(1)$

$\mathrm{S}(1)-\mathrm{Ru}(1)-\mathrm{P}(1)$

$\mathrm{P}(1)-\mathrm{S}(1)-\mathrm{Ru}(1)$

$\mathrm{C}(1)-\mathrm{P}(1)-\mathrm{C}(2)$

$C(2)-P(1)-C(8)$

$C(2)-P(1)-S(1)$

$\mathrm{C}(1)-\mathrm{P}(1)-\mathrm{Ru}(1)$

$\mathrm{C}(8)-\mathrm{P}(1)-\mathrm{Ru}(1)$

$\mathrm{C}(1)-\mathrm{P}(2)-\mathrm{C}(14)$

$C(14)-P(2)-C(20)$

$\mathrm{C}(14)-\mathrm{P}(2)-\mathrm{S}(2)$

$C(26)-P(3)-C(32)$

$C(32)-P(3)-C(38)$

$\mathrm{C}(32)-\mathrm{P}(3)-\mathrm{Ru}(1)$

$C(44)-P(4)-C(50)$

$C(50)-P(4)-C(56)$

$\mathrm{C}(50)-\mathrm{P}(4)-\mathrm{Ru}(1)$

$P(1)-C(1)-P(2)$

$P(2)-C(1)-R u(1)$

$\mathrm{P}(2)-\mathrm{C}(1)-\mathrm{H}(1)$

$C(7)-C(2)-C(3)$

$C(3)-C(2)-P(1)$

$\mathrm{C}(2)-\mathrm{C}(3)-\mathrm{Ru}(1)$

$C(5)-C(4)-C(3)$

$\mathrm{C}(3)-\mathrm{C}(4)-\mathrm{H}(4)$

$\mathrm{C}(6)-\mathrm{C}(5)-\mathrm{H}(5)$

$C(5)-C(6)-C(7)$

$\mathrm{C}(7)-\mathrm{C}(6)-\mathrm{H}(6)$

$\mathrm{C}(6)-\mathrm{C}(7)-\mathrm{H}(7)$

$C(9)-C(8)-C(13)$

$\mathrm{C}(13)-\mathrm{C}(8)-\mathrm{P}(1)$

$\mathrm{C}(8)-\mathrm{C}(9)-\mathrm{H}(9)$

$C(11)-C(10)-C(9)$

$\mathrm{C}(9)-\mathrm{C}(10)-\mathrm{H}(10)$

$\mathrm{C}(10)-\mathrm{C}(11)-\mathrm{H}(11)$

$C(13)-C(12)-C(11)$

$\mathrm{C}(11)-\mathrm{C}(12)-\mathrm{H}(12)$

$\mathrm{C}(8)-\mathrm{C}(13)-\mathrm{H}(13)$

C (15) - C (14)-C(19)

$C(19)-C(14)-P(2)$

$\mathrm{C}(16)-\mathrm{C}(15)-\mathrm{H}(15)$

$C(17)-C(16)-C(15)$

$\mathrm{C}(15)-\mathrm{C}(16)-\mathrm{H}(16)$

$\mathrm{C}(16)-\mathrm{C}(17)-\mathrm{H}(17)$

$C(17)-C(18)-C(19)$

$\mathrm{C}(19)-\mathrm{C}(18)-\mathrm{H}(18)$

$\mathrm{C}(18)-\mathrm{C}(19)-\mathrm{H}(19)$

$C(21)-C(20)-C(25)$

$\mathrm{C}(25)-\mathrm{C}(20)-\mathrm{P}(2)$

$\mathrm{C}(20)-\mathrm{C}(21)-\mathrm{H}(21)$

$C(23)-C(22)-C(21)$

$\mathrm{C}(21)-\mathrm{C}(22)-\mathrm{H}(22)$

$\mathrm{C}(22)-\mathrm{C}(23)-\mathrm{H}(23)$

$C(23)-C(24)-C(25)$

$\mathrm{C}(25)-\mathrm{C}(24)-\mathrm{H}(24)$

$\mathrm{C}(24)-\mathrm{C}(25)-\mathrm{H}(25)$

$C(31)-C(26)-C(27)$
0.9900

0.9900
$95.3(1)$

$86.4(1)$

$103.76(4)$

$75.1(1)$

$166.29(4)$

$77.4(1)$

$100.89(4)$

$63.6(1)$

$126.83(4)$

$45.05(4)$

$72.32(5)$

$102.8(2)$

$109.5(2)$

$107.4(2)$

$56.7(2)$

$172.8(2)$

$106.5(2)$

$105.6(2)$

112.1 (2)

$97.9(2)$

$96.9(2)$

$125.6(2)$

$104.2(2)$

$97.0(2)$

$121.2(2)$

$119.4(2)$

$94.2(2)$

120.3

$125.5(5)$

$107.0(3)$

$111.1(3)$

$121.6(5)$

119.2

119.3

$119.9(4)$

120.0

121.3

$120.0(5)$

$120.2(4)$

119.8

$119.6(6)$

120.2

119.7

$119.4(6)$

120.3

120.0

119.3 (5)

$120.5(4)$

120.1

$120.8(6)$

119.6

119.9

119.7 (5)

120.2

119.9

119.7 (5)

$119.3(4)$

119.9

119.9 (5)

120.0

119.8

$120.0(5)$

120.0

120.2

117.7(5) 


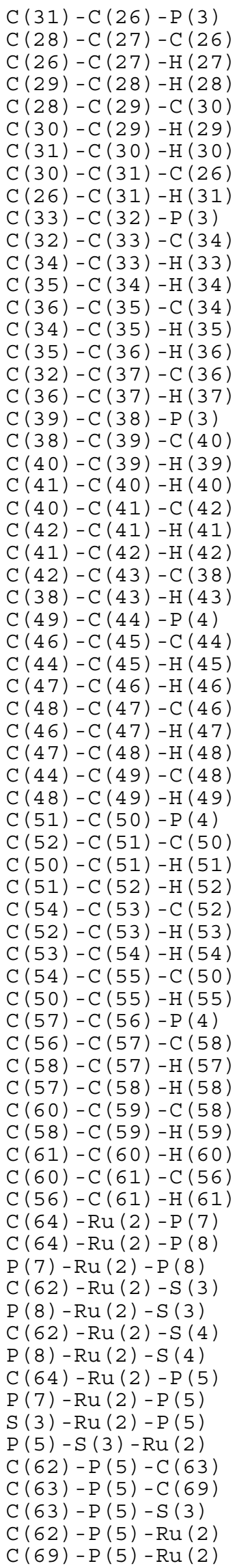

\begin{tabular}{|c|c|}
\hline $122.1(4)$ & $C(27)-C(26)-P(3)$ \\
\hline $119.8(5)$ & $\mathrm{C}(28)-\mathrm{C}(27)-\mathrm{H}(27)$ \\
\hline 20.1 & $C(29)-C(28)-C(27)$ \\
\hline 18.9 & $\mathrm{C}(27)-\mathrm{C}(28)-\mathrm{H}(28)$ \\
\hline $18.5(6)$ & $\mathrm{C}(28)-\mathrm{C}(29)-\mathrm{H}(29)$ \\
\hline 20.8 & $C(31)-C(30)-C(29)$ \\
\hline 19.7 & $\mathrm{C}(29)-\mathrm{C}(30)-\mathrm{H}(30)$ \\
\hline $21.0(5)$ & $\mathrm{C}(30)-\mathrm{C}(31)-\mathrm{H}(31)$ \\
\hline 9.5 & $C(33)-C(32)-C(37)$ \\
\hline $9.3(4)$ & $C(37)-C(32)-P(3)$ \\
\hline $.0(5)$ & $\mathrm{C}(32)-\mathrm{C}(33)-\mathrm{H}(33)$ \\
\hline .5 & $C(35)-C(34)-C(33)$ \\
\hline 0.1 & $\mathrm{C}(33)-\mathrm{C}(34)-\mathrm{H}(34)$ \\
\hline $20.0(5)$ & $\mathrm{C}(36)-\mathrm{C}(35)-\mathrm{H}(35)$ \\
\hline 20.0 & $C(35)-C(36)-C(37)$ \\
\hline 19.8 & $\mathrm{C}(37)-\mathrm{C}(36)-\mathrm{H}(36)$ \\
\hline $20.3(5)$ & $\mathrm{C}(32)-\mathrm{C}(37)-\mathrm{H}(37)$ \\
\hline 19.9 & $C(39)-C(38)-C(43)$ \\
\hline $25.9(4)$ & $C(43)-C(38)-P(3)$ \\
\hline $20.7(6)$ & $\mathrm{C}(38)-\mathrm{C}(39)-\mathrm{H}(39)$ \\
\hline 19.6 & $C(41)-C(40)-C(39)$ \\
\hline 9.6 & $\mathrm{C}(39)-\mathrm{C}(40)-\mathrm{H}(40)$ \\
\hline $9.5(5)$ & $\mathrm{C}(40)-\mathrm{C}(41)-\mathrm{H}(41)$ \\
\hline 0.3 & $C(41)-C(42)-C(43)$ \\
\hline .0 & $\mathrm{C}(43)-\mathrm{C}(42)-\mathrm{H}(42)$ \\
\hline $120.9(6)$ & $\mathrm{C}(42)-\mathrm{C}(43)-\mathrm{H}(43)$ \\
\hline 19.6 & $C(49)-C(44)-C(45)$ \\
\hline $24.7(5)$ & $\mathrm{C}(45)-\mathrm{C}(44)-\mathrm{P}(4)$ \\
\hline $121.5(6)$ & $\mathrm{C}(46)-\mathrm{C}(45)-\mathrm{H}(45)$ \\
\hline 19.2 & $C(47)-C(46)-C(45)$ \\
\hline 20.2 & $\mathrm{C}(45)-\mathrm{C}(46)-\mathrm{H}(46)$ \\
\hline $20.3(6)$ & $\mathrm{C}(48)-\mathrm{C}(47)-\mathrm{H}(47)$ \\
\hline 9.9 & $C(47)-C(48)-C(49)$ \\
\hline 9.4 & $\mathrm{C}(49)-\mathrm{C}(48)-\mathrm{H}(48)$ \\
\hline $9.4(6)$ & $\mathrm{C}(44)-\mathrm{C}(49)-\mathrm{H}(49)$ \\
\hline .3 & $C(51)-C(50)-C(55)$ \\
\hline $18.7(4)$ & $C(55)-C(50)-P(4)$ \\
\hline $21.4(5)$ & $\mathrm{C}(52)-\mathrm{C}(51)-\mathrm{H}(51)$ \\
\hline 19.3 & $C(51)-C(52)-C(53)$ \\
\hline 19.8 & $\mathrm{C}(53)-\mathrm{C}(52)-\mathrm{H}(52)$ \\
\hline $19.1(5)$ & $\mathrm{C}(54)-\mathrm{C}(53)-\mathrm{H}(53)$ \\
\hline 20.5 & $C(53)-C(54)-C(55)$ \\
\hline 19.6 & $\mathrm{C}(55)-\mathrm{C}(54)-\mathrm{H}(54)$ \\
\hline $0.2(6)$ & $\mathrm{C}(54)-\mathrm{C}(55)-\mathrm{H}(55)$ \\
\hline 9.9 & $C(57)-C(56)-C(61)$ \\
\hline $4.5(4)$ & $C(61)-C(56)-P(4)$ \\
\hline $4(5)$ & $\mathrm{C}(56)-\mathrm{C}(57)-\mathrm{H}(57)$ \\
\hline 18.8 & $C(57)-C(58)-C(59)$ \\
\hline 20.2 & $\mathrm{C}(59)-\mathrm{C}(58)-\mathrm{H}(58)$ \\
\hline $119.4(5)$ & $\mathrm{C}(60)-\mathrm{C}(59)-\mathrm{H}(59)$ \\
\hline 20.3 & $C(61)-C(60)-C(59)$ \\
\hline 20.0 & $\mathrm{C}(59)-\mathrm{C}(60)-\mathrm{H}(60)$ \\
\hline $21.9(5)$ & $\mathrm{C}(60)-\mathrm{C}(61)-\mathrm{H}(61)$ \\
\hline 19.1 & $C(64)-\operatorname{Ru}(2)-C(62)$ \\
\hline $.2(1)$ & $\mathrm{C}(62)-\mathrm{Ru}(2)-\mathrm{P}(7)$ \\
\hline $.3(1)$ & $\mathrm{C}(62)-\mathrm{Ru}(2)-\mathrm{P}(8)$ \\
\hline $3.29(5)$ & $C(64)-R u(2)-S(3)$ \\
\hline (1) & $P(7)-R u(2)-S(3)$ \\
\hline $86.90(4)$ & $\mathrm{C}(64)-\mathrm{Ru}(2)-\mathrm{S}(4)$ \\
\hline $7.6(1)$ & $\mathrm{P}(7)-\mathrm{Ru}(2)-\mathrm{S}(4)$ \\
\hline $5.16(4)$ & $S(3)-\operatorname{Ru}(2)-S(4)$ \\
\hline $53.4(1)$ & $C(62)-R u(2)-P(5)$ \\
\hline $23.79(4)$ & $\mathrm{P}(8)-\mathrm{Ru}(2)-\mathrm{P}(5)$ \\
\hline $4.66(4)$ & $S(4)-R u(2)-P(5)$ \\
\hline $2.44(5)$ & $P(6)-S(4)-R u(2)$ \\
\hline $03.3(2)$ & $C(62)-P(5)-C(69)$ \\
\hline $1.1(2)$ & $C(62)-P(5)-S(3)$ \\
\hline $5.9(2)$ & $C(69)-P(5)-S(3)$ \\
\hline (2) & $C(63)-P(5)-R u(2)$ \\
\hline 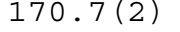 & $\mathrm{S}(3)-\mathrm{P}(5)-\mathrm{Ru}(2$ \\
\hline
\end{tabular}

$119.6(4)$

120.1

$122.1(6)$

118.9

120.8

$120.6(6)$

119.7

119.5

$118.4(5)$

$122.2(4)$

119.5

$119.8(5)$

120.1

120.0

$120.4(5)$

119.8

119.9

$118.0(5)$

$116.1(4)$

119.6

$120.8(6)$

119.6

120.3

$120.1(6)$

120.0

119.6

$118.1(5)$

$116.9(4)$

119.2

$119.5(6)$

120.2

119.9

$121.2(6)$

119.4

120.3

$118.2(5)$

$122.9(4)$

119.3

$120.3(6)$

119.8

120.5

120.7 (6)

119.6

119.9

$116.8(5)$

$118.7(4)$

118.8

119.5 (5)

120.2

120.3

$120.0(5)$

120.0

119.1

$86.6(2)$

$95.2(1)$

$160.9(1)$

$84.8(1)$

$168.44(4)$

$163.6(1)$

$97.75(4)$

$86.58(4)$

$40.2(1)$

$126.87(4)$

$100.85(4)$

$79.96(5)$

$117.9(2)$

$102.1(2)$

$115.1(2)$

$77.9(2)$

$62.90(5)$ 
$108.6(2)$

$102.4(2)$

$111.1(2)$

$100.4(2)$

$97.8(2)$

$120.6(2)$

$103.0(3)$

$100.4(2)$

$119.8(2)$

$119.7(3)$

$94.8(2)$

120.1

$124.0(4)$

$106.8(3)$

$111.4(3)$

$121.1(5)$

119.5

119.1

119.1 (5)

120.5

120.6

$119.9(5)$

$119.7(4)$

119.7

$120.8(7)$

119.6

120.4

$121.0(7)$

119.5

120.8

$118.5(5)$

$121.5(4)$

119.5

$119.1(6)$

120.5

119.5

$119.0(7)$

120.5

119.3

$118.8(5)$

$123.4(4)$

120.3

$120.9(6)$

119.6

120.0

$120.4(6)$

119.8

119.7

$117.5(4)$

$118.1(4)$

119.2

$120.2(5)$

119.9

120.1

$119.6(5)$

120.2

119.3

$118.0(5)$

$123.2(4)$

119.8

$122.0(6)$

119.0

120.9

$120.3(5)$

119.8

119.5

$118.0(4)$

$120.3(4)$

119.5

$120.4(5)$
$\mathrm{C}(62)-\mathrm{P}(6)-\mathrm{C}(75)$

$C(62)-P(6)-S(4)$

$C(75)-P(6)-S(4)$

$C(93)-P(7)-C(99)$

$\mathrm{C}(93)-\mathrm{P}(7)-\mathrm{Ru}(2)$

$\mathrm{C}(99)-\mathrm{P}(7)-\mathrm{Ru}(2)$

$C(105)-P(8)-C(117)$

$C(105)-P(8)-R u(2)$

$\mathrm{C}(117)-\mathrm{P}(8)-\mathrm{Ru}(2)$

$P(5)-C(62)-R u(2)$

$\mathrm{P}(5)-\mathrm{C}(62)-\mathrm{H}(62)$

$\mathrm{Ru}(2)-\mathrm{C}(62)-\mathrm{H}(62)$

$\mathrm{C}(68)-\mathrm{C}(63)-\mathrm{P}(5)$

$C(63)-C(64)-C(65)$

$C(65)-C(64)-\operatorname{Ru}(2)$

$\mathrm{C}(66)-\mathrm{C}(65)-\mathrm{H}(65)$

$C(65)-C(66)-C(67)$

$\mathrm{C}(67)-\mathrm{C}(66)-\mathrm{H}(66)$

$\mathrm{C}(68)-\mathrm{C}(67)-\mathrm{H}(67)$

$C(67)-C(68)-C(63)$

$\mathrm{C}(63)-\mathrm{C}(68)-\mathrm{H}(68)$

$C(70)-C(69)-P(5)$

$C(69)-C(70)-C(71)$

$\mathrm{C}(71)-\mathrm{C}(70)-\mathrm{H}(70)$

$\mathrm{C}(72)-\mathrm{C}(71)-\mathrm{H}(71)$

$C(71)-C(72)-C(73)$

$\mathrm{C}(73)-\mathrm{C}(72)-\mathrm{H}(72)$

$\mathrm{C}(72)-\mathrm{C}(73)-\mathrm{H}(73)$

$C(73)-C(74)-C(69)$

$\mathrm{C}(69)-\mathrm{C}(74)-\mathrm{H}(74)$

$\mathrm{C}(80)-\mathrm{C}(75)-\mathrm{P}(6)$

$C(75)-C(76)-C(77)$

$\mathrm{C}(77)-\mathrm{C}(76)-\mathrm{H}(76)$

$\mathrm{C}(78)-\mathrm{C}(77)-\mathrm{H}(77)$

$\mathrm{C}(77)-\mathrm{C}(78)-\mathrm{C}(79)$

$\mathrm{C}(79)-\mathrm{C}(78)-\mathrm{H}(78)$

$\mathrm{C}(80)-\mathrm{C}(79)-\mathrm{H}(79)$

$C(79)-C(80)-C(75)$

$\mathrm{C}(75)-\mathrm{C}(80)-\mathrm{H}(80)$

$C(86)-C(81)-P(6)$

$C(81)-C(82)-C(83)$

$\mathrm{C}(83)-\mathrm{C}(82)-\mathrm{H}(82)$

$\mathrm{C}(84)-\mathrm{C}(83)-\mathrm{H}(83)$

$C(83)-C(84)-C(85)$

$\mathrm{C}(85)-\mathrm{C}(84)-\mathrm{H}(84)$

$\mathrm{C}(86)-\mathrm{C}(85)-\mathrm{H}(85)$

$C(85)-C(86)-C(81)$

$\mathrm{C}(81)-\mathrm{C}(86)-\mathrm{H}(86)$

$\mathrm{C}(92)-\mathrm{C}(87)-\mathrm{P}(7)$

$C(89)-C(88)-C(87)$

$\mathrm{C}(87)-\mathrm{C}(88)-\mathrm{H}(88)$

$\mathrm{C}(88)-\mathrm{C}(89)-\mathrm{H}(89)$

$C(91)-C(90)-C(89)$

$\mathrm{C}(89)-\mathrm{C}(90)-\mathrm{H}(90)$

$\mathrm{C}(90)-\mathrm{C}(91)-\mathrm{H}(91)$

$C(87)-C(92)-C(91)$

$\mathrm{C}(91)-\mathrm{C}(92)-\mathrm{H}(92)$

$\mathrm{C}(98)-\mathrm{C}(93)-\mathrm{P}(7)$

$C(95)-C(94)-C(93)$

$\mathrm{C}(93)-\mathrm{C}(94)-\mathrm{H}(94)$

$\mathrm{C}(96)-\mathrm{C}(95)-\mathrm{H}(95)$

$C(95)-C(96)-C(97)$

$\mathrm{C}(97)-\mathrm{C}(96)-\mathrm{H}(96)$

$\mathrm{C}(96)-\mathrm{C}(97)-\mathrm{H}(97)$

$\mathrm{C}(93)-\mathrm{C}(98)-\mathrm{C}(97)$

$\mathrm{C}(97)-\mathrm{C}(98)-\mathrm{H}(98)$

$C(104)-C(99)-P(7)$

$C(101)-C(100)-C(99)$

$\mathrm{C}(99)-\mathrm{C}(100)-\mathrm{H}(100)$

$\mathrm{C}(102)-\mathrm{C}(101)-\mathrm{H}(101)$
$114.9(2)$

$107.2(2)$

$112.6(2)$

$99.8(2)$

$118.0(2)$

$116.3(2)$

$96.6(2)$

$120.0(2)$

$113.0(2)$

84.1 (2)

120.1

91.1

$129.1(4)$

$115.4(4)$

$133.0(4)$

119.5

$121.8(5)$

119.1

120.5

$118.7(5)$

120.6

$120.3(5)$

$120.6(7)$

119.7

119.6

$119.2(6)$

120.4

119.5

$118.4(6)$

120.8

$120.0(4)$

$120.9(5)$

119.5

120.5

$121.0(6)$

119.5

120.5

$121.4(6)$

119.3

$117.8(4)$

$119.3(6)$

120.3

119.6

$119.9(6)$

120.0

119.8

120.7 (5)

119.7

$124.3(4)$

$121.5(5)$

119.2

119.9

119.7 (5)

120.1

120.2

$121.4(5)$

119.3

$118.8(4)$

$120.4(5)$

119.8

119.0

118.2 (5)

120.9

119.8

$121.0(5)$

119.5

$121.5(4)$

$121.0(5)$

119.5

119.8 


\begin{tabular}{|c|c|c|c|}
\hline $\mathrm{C}(100)-\mathrm{C}(101)-\mathrm{H}(101)$ & 119.8 & $C(103)-C(102)-C(101)$ & $119.3(5)$ \\
\hline $\mathrm{C}(103)-\mathrm{C}(102)-\mathrm{H}(102)$ & 120.3 & $\mathrm{C}(101)-\mathrm{C}(102)-\mathrm{H}(102)$ & 120.3 \\
\hline$C(102)-C(103)-C(104)$ & $120.2(5)$ & $\mathrm{C}(102)-\mathrm{C}(103)-\mathrm{H}(103)$ & 119.9 \\
\hline $\mathrm{C}(104)-\mathrm{C}(103)-\mathrm{H}(103)$ & 119.9 & $C(99)-C(104)-C(103)$ & $121.0(5)$ \\
\hline $\mathrm{C}(99)-\mathrm{C}(104)-\mathrm{H}(104)$ & 119.5 & $\mathrm{C}(103)-\mathrm{C}(104)-\mathrm{H}(104)$ & 119.5 \\
\hline$C(106)-C(105)-C(110)$ & $118.1(5)$ & $C(106)-C(105)-P(8)$ & $120.4(4)$ \\
\hline$C(110)-C(105)-P(8)$ & $121.4(4)$ & $C(105)-C(106)-C(107)$ & $121.4(5)$ \\
\hline $\mathrm{C}(105)-\mathrm{C}(106)-\mathrm{H}(106)$ & 119.3 & $\mathrm{C}(107)-\mathrm{C}(106)-\mathrm{H}(106)$ & 119.3 \\
\hline$C(108)-C(107)-C(106)$ & $120.0(6)$ & $\mathrm{C}(108)-\mathrm{C}(107)-\mathrm{H}(107)$ & 120.0 \\
\hline $\mathrm{C}(106)-\mathrm{C}(107)-\mathrm{H}(107)$ & 120.0 & $C(109)-C(108)-C(107)$ & $119.0(5)$ \\
\hline $\mathrm{C}(109)-\mathrm{C}(108)-\mathrm{H}(108)$ & 120.5 & $\mathrm{C}(107)-\mathrm{C}(108)-\mathrm{H}(108)$ & 120.5 \\
\hline$C(108)-C(109)-C(110)$ & $121.4(6)$ & $\mathrm{C}(108)-\mathrm{C}(109)-\mathrm{H}(109)$ & 119.3 \\
\hline $\mathrm{C}(110)-\mathrm{C}(109)-\mathrm{H}(109)$ & 119.3 & $C(109)-C(110)-C(105)$ & $120.1(6)$ \\
\hline $\mathrm{C}(109)-\mathrm{C}(110)-\mathrm{H}(110)$ & 119.9 & $\mathrm{C}(105)-\mathrm{C}(110)-\mathrm{H}(110)$ & 119.9 \\
\hline$C(116)-C(111)-C(112)$ & $118.9(6)$ & $C(116)-C(111)-P(8)$ & $117.4(5)$ \\
\hline$C(112)-C(111)-P(8)$ & $123.7(5)$ & $C(113)-C(112)-C(111)$ & $123.0(8)$ \\
\hline $\mathrm{C}(113)-\mathrm{C}(112)-\mathrm{H}(112)$ & 118.5 & $\mathrm{C}(111)-\mathrm{C}(112)-\mathrm{H}(112)$ & 118.5 \\
\hline$C(114)-C(113)-C(112)$ & $118.6(8)$ & $\mathrm{C}(114)-\mathrm{C}(113)-\mathrm{H}(113)$ & 120.7 \\
\hline $\mathrm{C}(112)-\mathrm{C}(113)-\mathrm{H}(113)$ & 120.7 & $C(113)-C(114)-C(115)$ & $121.4(7)$ \\
\hline $\mathrm{C}(113)-\mathrm{C}(114)-\mathrm{H}(114)$ & 119.3 & $\mathrm{C}(115)-\mathrm{C}(114)-\mathrm{H}(114)$ & 119.3 \\
\hline$C(114)-C(115)-C(116)$ & $119.4(8)$ & $\mathrm{C}(114)-\mathrm{C}(115)-\mathrm{H}(115)$ & 120.3 \\
\hline $\mathrm{C}(116)-\mathrm{C}(115)-\mathrm{H}(115)$ & 120.3 & $C(111)-C(116)-C(115)$ & $118.7(7)$ \\
\hline $\mathrm{C}(111)-\mathrm{C}(116)-\mathrm{H}(116)$ & 120.7 & $\mathrm{C}(115)-\mathrm{C}(116)-\mathrm{H}(116)$ & 120.7 \\
\hline$C(118)-C(117)-C(122)$ & $118.8(5)$ & $C(118)-C(117)-P(8)$ & $124.2(4)$ \\
\hline$C(122)-C(117)-P(8)$ & $116.9(4)$ & $C(117)-C(118)-C(119)$ & $120.2(5)$ \\
\hline $\mathrm{C}(117)-\mathrm{C}(118)-\mathrm{H}(118)$ & 119.9 & $\mathrm{C}(119)-\mathrm{C}(118)-\mathrm{H}(118)$ & 119.9 \\
\hline$C(120)-C(119)-C(118)$ & $120.5(5)$ & $\mathrm{C}(120)-\mathrm{C}(119)-\mathrm{H}(119)$ & 119.8 \\
\hline $\mathrm{C}(118)-\mathrm{C}(119)-\mathrm{H}(119)$ & 119.8 & $C(119)-C(120)-C(121)$ & $119.7(5)$ \\
\hline $\mathrm{C}(119)-\mathrm{C}(120)-\mathrm{H}(120)$ & 120.1 & $\mathrm{C}(121)-\mathrm{C}(120)-\mathrm{H}(120)$ & 120.1 \\
\hline$C(120)-C(121)-C(122)$ & $120.2(5)$ & $\mathrm{C}(120)-\mathrm{C}(121)-\mathrm{H}(121)$ & 119.9 \\
\hline $\mathrm{C}(122)-\mathrm{C}(121)-\mathrm{H}(121)$ & 119.9 & $C(121)-C(122)-C(117)$ & $120.6(5)$ \\
\hline $\mathrm{C}(121)-\mathrm{C}(122)-\mathrm{H}(122)$ & 119.7 & $\mathrm{C}(117)-\mathrm{C}(122)-\mathrm{H}(122)$ & 119.7 \\
\hline$C(124) \# 4-C(123)-C l(1)$ & $111.3(5)$ & $\mathrm{C}(124) \# 4-\mathrm{C}(123)-\mathrm{H}(12 \mathrm{~A})$ & 109.4 \\
\hline $\mathrm{Cl}(1)-\mathrm{C}(123)-\mathrm{H}(12 \mathrm{~A})$ & 109.4 & $\mathrm{C}(124) \# 4-\mathrm{C}(123)-\mathrm{H}(12 \mathrm{~B})$ & 109.4 \\
\hline $\mathrm{Cl}(1)-\mathrm{C}(123)-\mathrm{H}(12 \mathrm{~B})$ & 109.4 & $\mathrm{H}(12 \mathrm{~A})-\mathrm{C}(123)-\mathrm{H}(12 \mathrm{~B})$ & 108.0 \\
\hline $\mathrm{C}(123) \# 4-\mathrm{C}(124)-\mathrm{Cl}(2) \# 4$ & $111.5(5)$ & $\mathrm{C}(123) \# 4-\mathrm{C}(124)-\mathrm{H}(12 \mathrm{C})$ & 109.3 \\
\hline $\mathrm{Cl}(2) \# 4-\mathrm{C}(124)-\mathrm{H}(12 \mathrm{C})$ & 109.3 & $\mathrm{C}(123) \# 4-\mathrm{C}(124)-\mathrm{H}(12 \mathrm{D})$ & 109.3 \\
\hline $\mathrm{Cl}(2) \# 4-\mathrm{C}(124)-\mathrm{H}(12 \mathrm{D})$ & 109.3 & $\mathrm{H}(12 \mathrm{C})-\mathrm{C}(124)-\mathrm{H}(12 \mathrm{D})$ & 108.0 \\
\hline$C(126)-C(125)-C 1(3)$ & $110(1)$ & $\mathrm{C}(126)-\mathrm{C}(125)-\mathrm{H}(12 \mathrm{E})$ & 109.7 \\
\hline $\mathrm{Cl}(3)-\mathrm{C}(125)-\mathrm{H}(12 \mathrm{E})$ & 109.7 & $\mathrm{C}(126)-\mathrm{C}(125)-\mathrm{H}(12 \mathrm{~F})$ & 109.7 \\
\hline $\mathrm{Cl}(3)-\mathrm{C}(125)-\mathrm{H}(12 \mathrm{~F})$ & 109.7 & $\mathrm{H}(12 \mathrm{E})-\mathrm{C}(125)-\mathrm{H}(12 \mathrm{~F})$ & 108.2 \\
\hline$C(125)-C(126)-C 1(4) \# 2$ & $110(1)$ & $\mathrm{C}(125)-\mathrm{C}(126)-\mathrm{H}(12 \mathrm{G})$ & 109.7 \\
\hline $\mathrm{Cl}(4) \# 2-\mathrm{C}(126)-\mathrm{H}(12 \mathrm{G})$ & 109.7 & $\mathrm{C}(125)-\mathrm{C}(126)-\mathrm{H}(12 \mathrm{H})$ & 109.7 \\
\hline $\mathrm{Cl}(4) \# 2-\mathrm{C}(126)-\mathrm{H}(12 \mathrm{H})$ & 109.7 & $\mathrm{H}(12 \mathrm{G})-\mathrm{C}(126)-\mathrm{H}(12 \mathrm{H})$ & 108.2 \\
\hline
\end{tabular}

Estimated standard deviations are given in the parenthesis. Symmetry operators : :
1: $\mathrm{x}, \mathrm{y}, \mathrm{z}$
$4: x,-y-1 / 2, z-1 / 2$
$2:-x, y+1 / 2,-z+1 / 2 \quad 3:-x,-y,-z$ 
Table S2-4. Anisotropic displacement parameters $\left(A^{\wedge} 2 \times 10^{\wedge} 3\right.$ ) for 3

\begin{tabular}{|c|c|c|c|c|c|c|}
\hline atom & U11 & $\mathrm{U} 22$ & U33 & U2 3 & U13 & U12 \\
\hline 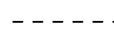 & & & 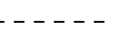 & . & - - & ------ \\
\hline $\operatorname{Ru}(1)$ & $17(1)$ & $15(1)$ & $14(1)$ & $0(1)$ & $1(1)$ & $1(1)$ \\
\hline$S(1)$ & $17(1)$ & $19(1)$ & $19(1)$ & $-2(1)$ & $1(1)$ & $3(1)$ \\
\hline$S(2)$ & $22(1)$ & $18(1)$ & $15(1)$ & $-2(1)$ & $0(1)$ & $-1(1)$ \\
\hline$P(1)$ & $19(1)$ & $18(1)$ & $17(1)$ & $-2(1)$ & $0(1)$ & $1(1)$ \\
\hline$P(2)$ & $18(1)$ & $15(1)$ & $17(1)$ & $1(1)$ & $0(1)$ & $1(1)$ \\
\hline$P(3)$ & $17(1)$ & $17(1)$ & $18(1)$ & $2(1)$ & $1(1)$ & $2(1)$ \\
\hline P (4) & $21(1)$ & $15(1)$ & $19(1)$ & $0(1)$ & $-1(1)$ & $-1(1)$ \\
\hline$C(1)$ & $18(2)$ & $16(2)$ & $11(2)$ & $2(2)$ & $4(2)$ & $2(2)$ \\
\hline$C(2)$ & $17(2)$ & $24(2)$ & $21(2)$ & $-1(2)$ & $0(2)$ & $4(2)$ \\
\hline$C(3)$ & $14(2)$ & $21(2)$ & $21(2)$ & $2(2)$ & $0(2)$ & $1(2)$ \\
\hline$C(4)$ & $18(2)$ & $26(2)$ & $22(2)$ & $4(2)$ & $2(2)$ & $1(2)$ \\
\hline$C(5)$ & $25(2)$ & $36(3)$ & $20(2)$ & $7(2)$ & $2(2)$ & $-1(2)$ \\
\hline$C(6)$ & $30(3)$ & $42(3)$ & $12(2)$ & $0(2)$ & $-1(2)$ & $2(2)$ \\
\hline$C(7)$ & $24(2)$ & $27(2)$ & $19(2)$ & $-4(2)$ & $0(2)$ & $3(2)$ \\
\hline$C(8)$ & $30(3)$ & $21(2)$ & $23(2)$ & $-4(2)$ & $1(2)$ & $2(2)$ \\
\hline$C(9)$ & $36(3)$ & $21(2)$ & $28(3)$ & $1(2)$ & $3(2)$ & $5(2)$ \\
\hline$C(10)$ & $61(4)$ & $19(2)$ & $35(3)$ & $-4(2)$ & $15(3)$ & $10(3)$ \\
\hline$C(11)$ & $75(5)$ & $23(3)$ & $41(3)$ & $-10(2)$ & $17(3)$ & $-1(3)$ \\
\hline$C(12)$ & $58(4)$ & $37(3)$ & $44(4)$ & $-15(3)$ & $-2(3)$ & $-11(3)$ \\
\hline$C(13)$ & $46(3)$ & $31(3)$ & $35(3)$ & $-8(2)$ & $3(3)$ & $-2(2)$ \\
\hline$C(14)$ & $22(2)$ & $21(2)$ & $21(2)$ & $7(2)$ & $1(2)$ & $2(2)$ \\
\hline$C(15)$ & $24(2)$ & $37(3)$ & $24(3)$ & $-3(2)$ & $0(2)$ & $3(2)$ \\
\hline$C(16)$ & $23(3)$ & $60(4)$ & $34(3)$ & $1(3)$ & $9(2)$ & $5(3)$ \\
\hline $\mathrm{C}(17)$ & $24(3)$ & $53(4)$ & $50(4)$ & $1(3)$ & $-1(3)$ & $-5(3)$ \\
\hline$C(18)$ & $29(3)$ & $34(3)$ & $42(3)$ & $-5(3)$ & $-8(2)$ & $-3(2)$ \\
\hline$C(19)$ & $22(2)$ & $31(3)$ & $30(3)$ & $-2(2)$ & $-2(2)$ & $-3(2)$ \\
\hline$C(20)$ & $22(2)$ & $18(2)$ & $21(2)$ & $1(2)$ & $4(2)$ & $1(2)$ \\
\hline$C(21)$ & $23(2)$ & $24(2)$ & $23(2)$ & $-1(2)$ & $2(2)$ & $0(2)$ \\
\hline$C(22)$ & $26(2)$ & $28(3)$ & $27(3)$ & $4(2)$ & $1(2)$ & $6(2)$ \\
\hline$C(23)$ & $34(3)$ & $24(3)$ & $40(3)$ & $10(2)$ & $3(2)$ & $10(2)$ \\
\hline$C(24)$ & $38(3)$ & $22(2)$ & $36(3)$ & $-5(2)$ & $6(2)$ & $3(2)$ \\
\hline$C(25)$ & $31(3)$ & $24(2)$ & $29(3)$ & $2(2)$ & $-6(2)$ & $-2(2)$ \\
\hline$C(26)$ & $18(2)$ & $17(2)$ & $28(3)$ & $2(2)$ & $1(2)$ & $5(2)$ \\
\hline$C(27)$ & $34(3)$ & $30(3)$ & $33(3)$ & $-3(2)$ & $10(2)$ & $-2(2)$ \\
\hline $\mathrm{C}(28)$ & $27(3)$ & $34(3)$ & $57(4)$ & $-5(3)$ & $3(3)$ & $-6(2)$ \\
\hline$C(29)$ & $34(3)$ & $50(4)$ & $47(4)$ & $-13(3)$ & $-7(3)$ & $-8(3)$ \\
\hline$C(30)$ & $42(3)$ & $40(3)$ & $33(3)$ & $-9(3)$ & $0(3)$ & $-2(3)$ \\
\hline$C(31)$ & $28(2)$ & $29(3)$ & $26(3)$ & $0(2)$ & $-2(2)$ & $-3(2)$ \\
\hline$C(32)$ & $24(2)$ & $19(2)$ & $27(3)$ & $-1(2)$ & $3(2)$ & $3(2)$ \\
\hline$C(33)$ & $27(2)$ & $20(2)$ & $28(3)$ & $1(2)$ & $0(2)$ & $4(2)$ \\
\hline$C(34)$ & $29(3)$ & $26(3)$ & $39(3)$ & $0(2)$ & $9(2)$ & $8(2)$ \\
\hline$C(35)$ & $36(3)$ & $28(3)$ & $36(3)$ & $-3(2)$ & $11(2)$ & $6(2)$ \\
\hline$C(36)$ & $49(3)$ & $33(3)$ & $29(3)$ & $4(2)$ & $15(3)$ & $13(3)$ \\
\hline$C(37)$ & $35(3)$ & $25(3)$ & $27(3)$ & $4(2)$ & $7(2)$ & $10(2)$ \\
\hline$C(38)$ & $17(2)$ & $18(2)$ & $26(2)$ & $3(2)$ & $5(2)$ & $7(2)$ \\
\hline$C(39)$ & $44(3)$ & $24(3)$ & $27(3)$ & $6(2)$ & $2(2)$ & $3(2)$ \\
\hline$C(40)$ & $58(4)$ & $30(3)$ & $34(3)$ & $13(3)$ & $16(3)$ & $10(3)$ \\
\hline$C(41)$ & $35(3)$ & $20(3)$ & $67(4)$ & $19(3)$ & $20(3)$ & $4(2)$ \\
\hline$C(42)$ & $49(4)$ & $18(2)$ & $55(4)$ & $2(3)$ & $-7(3)$ & $-3(2)$ \\
\hline$C(43)$ & $46(3)$ & $17(2)$ & $43(3)$ & $2(2)$ & $-5(3)$ & $0(2)$ \\
\hline$C(44)$ & $32(3)$ & $23(2)$ & $23(2)$ & $5(2)$ & $-3(2)$ & $-4(2)$ \\
\hline$C(45)$ & $25(3)$ & $40(3)$ & $33(3)$ & $0(2)$ & $-3(2)$ & $-10(2)$ \\
\hline$C(46)$ & $35(3)$ & $58(4)$ & $33(3)$ & $-1(3)$ & $9(2)$ & $-11(3)$ \\
\hline$C(47)$ & $48(4)$ & $59(4)$ & $32(3)$ & $15(3)$ & $-3(3)$ & $-16(3)$ \\
\hline$C(48)$ & $53(4)$ & $29(3)$ & $49(4)$ & $16(3)$ & $-9(3)$ & $-7(3)$ \\
\hline$C(49)$ & $55(4)$ & $23(2)$ & $32(3)$ & $7(2)$ & $-5(3)$ & $1(3)$ \\
\hline$C(50)$ & $28(2)$ & $21(2)$ & $20(2)$ & $-5(2)$ & $-9(2)$ & $5(2)$ \\
\hline$C(51)$ & $32(3)$ & $25(2)$ & $27(3)$ & $-4(2)$ & $-3(2)$ & $6(2)$ \\
\hline$C(52)$ & $49(3)$ & $38(3)$ & $23(3)$ & $-1(2)$ & $4(2)$ & $14(3)$ \\
\hline$C(53)$ & $69(4)$ & $29(3)$ & $30(3)$ & $-14(2)$ & $-13(3)$ & $18(3)$ \\
\hline $\mathrm{C}(54)$ & $60(4)$ & $21(3)$ & $37(3)$ & $-6(2)$ & $-20(3)$ & $1(3)$ \\
\hline$C(55)$ & $37(3)$ & $22(2)$ & $35(3)$ & $3(2)$ & $-5(2)$ & $-2(2)$ \\
\hline$C(56)$ & $26(2)$ & $17(2)$ & $23(2)$ & $-4(2)$ & $-4(2)$ & $1(2)$ \\
\hline$C(57)$ & $29(3)$ & $36(3)$ & $37(3)$ & $16(3)$ & $3(2)$ & $-3(2)$ \\
\hline
\end{tabular}




\begin{tabular}{|c|c|c|c|c|c|c|}
\hline$C(58)$ & $25(3)$ & $59(4)$ & $47(4)$ & $15(3)$ & $2(2)$ & $5(3)$ \\
\hline C (59) & $26(3)$ & $43(3)$ & $36(3)$ & $-2(3)$ & $-5(2)$ & $8(2)$ \\
\hline$C(60)$ & $30(3)$ & $28(3)$ & $26(3)$ & $7(2)$ & $-3(2)$ & $4(2)$ \\
\hline$C(61)$ & $23(2)$ & $28(2)$ & $22(2)$ & $-2(2)$ & $-2(2)$ & $-1(2)$ \\
\hline Ru (2) & 16 (1) & $17(1)$ & $15(1)$ & $2(1)$ & $0(1)$ & $1(1)$ \\
\hline$S(3)$ & $18(1)$ & $24(1)$ & $23(1)$ & $2(1)$ & $1(1)$ & $3(1)$ \\
\hline S (4) & $24(1)$ & $23(1)$ & $17(1)$ & $3(1)$ & $1(1)$ & $-3(1)$ \\
\hline$P(5)$ & $22(1)$ & $20(1)$ & $19(1)$ & $4(1)$ & $0(1)$ & $3(1)$ \\
\hline$P(6)$ & 19 (1) & $19(1)$ & $17(1)$ & $0(1)$ & $1(1)$ & $1(1)$ \\
\hline $\mathrm{P}(7)$ & $17(1)$ & $19(1)$ & $17(1)$ & $2(1)$ & $-1(1)$ & $3(1)$ \\
\hline $\mathrm{P}(8)$ & $20(1)$ & 19 (1) & $20(1)$ & $1(1)$ & $2(1)$ & $-2(1)$ \\
\hline$C(62)$ & $26(2)$ & $16(2)$ & $20(2)$ & $2(2)$ & $0(2)$ & $2(2)$ \\
\hline$C(63)$ & $22(2)$ & $25(2)$ & $14(2)$ & $-2(2)$ & $-2(2)$ & $2(2)$ \\
\hline$C(64)$ & $19(2)$ & $27(2)$ & $9(2)$ & $-4(2)$ & $-2(2)$ & $0(2)$ \\
\hline$C(65)$ & $18(2)$ & $26(2)$ & $24(2)$ & $2(2)$ & $-1(2)$ & $3(2)$ \\
\hline$C(66)$ & $28(3)$ & $34(3)$ & $19(2)$ & $-5(2)$ & $-1(2)$ & $2(2)$ \\
\hline$C(67)$ & $33(3)$ & $44(3)$ & $15(2)$ & $8(2)$ & $0(2)$ & $4(2)$ \\
\hline$C(68)$ & $24(2)$ & $30(3)$ & $22(2)$ & $9(2)$ & $0(2)$ & $3(2)$ \\
\hline$C(69)$ & $28(3)$ & $27(3)$ & $30(3)$ & $5(2)$ & $-7(2)$ & $-1(2)$ \\
\hline$C(70)$ & $67(5)$ & $41(4)$ & $97(7)$ & $32(4)$ & $42(5)$ & $29(4)$ \\
\hline$C(71)$ & $90(6)$ & $41(4)$ & $106(7)$ & $32(5)$ & $38(6)$ & $39(4)$ \\
\hline$C(72)$ & $85(6)$ & $28(3)$ & $66(5)$ & $10(3)$ & $-22(4)$ & $10(3)$ \\
\hline$C(73)$ & $60(5)$ & $41(4)$ & $72(5)$ & $23(4)$ & $-11(4)$ & $-17(3)$ \\
\hline$C(74)$ & $31(3)$ & $34(3)$ & $60(4)$ & $15(3)$ & $-3(3)$ & $0(2)$ \\
\hline$C(75)$ & $29(3)$ & $25(2)$ & $22(2)$ & $1(2)$ & $-5(2)$ & $7(2)$ \\
\hline$C(76)$ & $22(2)$ & $36(3)$ & $26(3)$ & $-5(2)$ & $0(2)$ & $3(2)$ \\
\hline$C(77)$ & $33(3)$ & $49(4)$ & $37(3)$ & $-12(3)$ & $1(2)$ & $14(3)$ \\
\hline$C(78)$ & $63(5)$ & $41(4)$ & $63(5)$ & $-8(3)$ & $15(4)$ & $26(3)$ \\
\hline$C(79)$ & $79(6)$ & $27(3)$ & $79(6)$ & $9(3)$ & $11(4)$ & $18(3)$ \\
\hline$C(80)$ & $47(3)$ & $24(3)$ & $66(5)$ & $10(3)$ & $27(3)$ & $7(3)$ \\
\hline$C(81)$ & $18(2)$ & $19(2)$ & $29(3)$ & $-1(2)$ & $5(2)$ & $2(2)$ \\
\hline$C(82)$ & $28(3)$ & $35(3)$ & $36(3)$ & $-5(2)$ & $9(2)$ & $-10(2)$ \\
\hline$C(83)$ & $29(3)$ & $45(3)$ & $47(4)$ & $-2(3)$ & $14(3)$ & $-11(3)$ \\
\hline$C(84)$ & $27(3)$ & $51(4)$ & $50(4)$ & $-6(3)$ & $-2(3)$ & $-10(3)$ \\
\hline$C(85)$ & $26(3)$ & $48(4)$ & $40(3)$ & $-6(3)$ & $-7(2)$ & $-1(2)$ \\
\hline$C(86)$ & $24(2)$ & $33(3)$ & $26(3)$ & $2(2)$ & $0(2)$ & $1(2)$ \\
\hline$C(87)$ & $18(2)$ & $19(2)$ & $17(2)$ & $3(2)$ & $-1(2)$ & $4(2)$ \\
\hline$C(88)$ & $21(2)$ & $28(3)$ & $34(3)$ & $-6(2)$ & $-3(2)$ & $5(2)$ \\
\hline$C(89)$ & $32(3)$ & $41(3)$ & $30(3)$ & $-11(2)$ & $-10(2)$ & $7(2)$ \\
\hline$C(90)$ & $33(3)$ & $43(3)$ & $26(3)$ & $9(2)$ & $-8(2)$ & $5(2)$ \\
\hline C (91) & $41(3)$ & $30(3)$ & $44(3)$ & $13(3)$ & $-15(3)$ & $5(2)$ \\
\hline C (92) & $31(3)$ & $22(2)$ & $34(3)$ & $2(2)$ & $-11(2)$ & $-2(2)$ \\
\hline$C(93)$ & $25(2)$ & $21(2)$ & $20(2)$ & $-1(2)$ & $-2(2)$ & $5(2)$ \\
\hline$C(94)$ & $34(3)$ & $30(3)$ & $28(3)$ & $-1(2)$ & $4(2)$ & $-2(2)$ \\
\hline$C(95)$ & $50(4)$ & $38(3)$ & $27(3)$ & $-12(2)$ & $4(3)$ & $-2(3)$ \\
\hline$C(96)$ & $51(4)$ & $27(3)$ & $34(3)$ & $-12(2)$ & $-5(3)$ & $0(2)$ \\
\hline$C(97)$ & $42(3)$ & $21(2)$ & $35(3)$ & $-2(2)$ & $-6(2)$ & $0(2)$ \\
\hline$C(98)$ & $30(3)$ & $27(3)$ & $20(2)$ & $0(2)$ & $-3(2)$ & $-1(2)$ \\
\hline C (99) & $19(2)$ & $23(2)$ & $19(2)$ & $-3(2)$ & $5(2)$ & $0(2)$ \\
\hline$C(100)$ & $21(2)$ & $27(2)$ & $20(2)$ & $3(2)$ & $2(2)$ & $5(2)$ \\
\hline$C(101)$ & $31(3)$ & $35(3)$ & $22(3)$ & $9(2)$ & $2(2)$ & $-1(2)$ \\
\hline$C(102)$ & $24(3)$ & $36(3)$ & $34(3)$ & $2(2)$ & $10(2)$ & $-6(2)$ \\
\hline$C(103)$ & $17(2)$ & $48(3)$ & $30(3)$ & $1(2)$ & $-1(2)$ & $1(2)$ \\
\hline$C(104)$ & $22(2)$ & $34(3)$ & $27(3)$ & $5(2)$ & $5(2)$ & $-1(2)$ \\
\hline$C(105)$ & $28(3)$ & $23(2)$ & $25(3)$ & $2(2)$ & $8(2)$ & $1(2)$ \\
\hline$C(106)$ & $33(3)$ & $31(3)$ & $24(3)$ & $4(2)$ & $7(2)$ & $7(2)$ \\
\hline$C(107)$ & $52(4)$ & $34(3)$ & $24(3)$ & $5(2)$ & $2(2)$ & $8(3)$ \\
\hline$C(108)$ & $62(4)$ & $29(3)$ & $28(3)$ & $11(2)$ & $12(3)$ & $15(3)$ \\
\hline$C(109)$ & $49(4)$ & $28(3)$ & $44(4)$ & $13(3)$ & $18(3)$ & $0(3)$ \\
\hline$C(110)$ & $35(3)$ & $30(3)$ & $42(3)$ & $7(2)$ & $10(3)$ & $-4(2)$ \\
\hline $\mathrm{C}(111)$ & $17(2)$ & $48(3)$ & $30(3)$ & $-12(2)$ & $5(2)$ & $-9(2)$ \\
\hline $\mathrm{C}(112)$ & $30(3)$ & $45(3)$ & $55(4)$ & $-25(3)$ & $17(3)$ & $-13(3)$ \\
\hline$C(113)$ & $65(5)$ & $62(5)$ & $63(5)$ & $-34(4)$ & $26(4)$ & $-27(4)$ \\
\hline$C(114)$ & $57(5)$ & $80(6)$ & $60(5)$ & $-40(5)$ & $21(4)$ & $-32(4)$ \\
\hline$C(115)$ & $39(4)$ & $103(7)$ & $33(4)$ & $-13(4)$ & $7(3)$ & $-18(4)$ \\
\hline$C(116)$ & $30(3)$ & $74(5)$ & $29(3)$ & $-10(3)$ & $5(2)$ & $-16(3)$ \\
\hline$C(117)$ & $24(2)$ & $21(2)$ & $23(2)$ & $4(2)$ & $5(2)$ & $-3(2)$ \\
\hline$C(118)$ & $22(2)$ & $32(3)$ & $25(3)$ & $-1(2)$ & $2(2)$ & $-4(2)$ \\
\hline$C(119)$ & $25(3)$ & $41(3)$ & $38(3)$ & $0(3)$ & $-7(2)$ & $2(2)$ \\
\hline$C(120)$ & $20(2)$ & $51(4)$ & $44(3)$ & $-6(3)$ & $10(2)$ & $3(2)$ \\
\hline
\end{tabular}




$\begin{array}{lllllll}C(121) & 32(3) & 48(3) & 28(3) & -11(3) & 4(2) & 3(2) \\ \mathrm{C}(122) & 26(2) & 41(3) & 23(3) & -2(2) & 1(2) & -1(2) \\ \mathrm{Cl}(1) & 52(1) & 65(1) & 46(1) & 1(1) & -5(1) & 15(1) \\ \mathrm{Cl}(2) & 84(2) & 62(1) & 71(1) & -25(1) & 4(1) & 8(1) \\ \mathrm{C}(123) & 58(4) & 38(3) & 43(4) & -3(3) & -4(3) & 7(3) \\ \mathrm{C}(124) & 64(5) & 46(4) & 47(4) & 5(3) & -10(3) & 0(3) \\ \mathrm{Cl}(3) & 152(3) & 64(2) & 94(2) & -19(1) & 42(2) & -37(2) \\ \mathrm{Cl}(4) & 107(2) & 118(2) & 237(5) & -100(3) & 109(3) & -49(2) \\ \mathrm{C}(125) & 160(20) & 170(20) & 90(10) & 10(10) & -10(10) & -30(10) \\ \mathrm{C}(126) & 120(10) & 104(9) & 101(9) & 31(8) & 31(8) & 23(8) \\ -\end{array}$

The anisotropic displacement factor exponent takes the form $2 \mathrm{pi}^{\wedge} 2\left[\mathrm{~h}^{\wedge} 2 \mathrm{a}^{\star \wedge} 2 \mathrm{U}(11)+\ldots+2 \mathrm{hka} \mathrm{H}^{*} \mathrm{~b} \mathrm{U}(12)\right]$ 
Table S2-5. Hydrogen Coordinates (A $\times 10^{\wedge} 4$ ) and equivalent isotropic displacement parameters $\left(A^{\wedge} 2 \times 10^{\wedge} 3\right)$ for 3

\begin{tabular}{|c|c|c|c|c|}
\hline atom & $\mathrm{x}$ & Y & $\mathrm{z}$ & $\mathrm{U}(\mathrm{eq})$ \\
\hline $\mathrm{H}(1)$ & -3444 & -336 & -2356 & 18 \\
\hline $\mathrm{H}(4)$ & -4592 & -1556 & -1461 & 26 \\
\hline $\mathrm{H}(5)$ & -4651 & -1441 & -258 & 32 \\
\hline $\mathrm{H}(6)$ & -4603 & -709 & 221 & 33 \\
\hline $\mathrm{H}(7)$ & -4537 & -67 & -522 & 28 \\
\hline $\mathrm{H}(9)$ & -5600 & 617 & -1999 & 34 \\
\hline $\mathrm{H}(10)$ & -5698 & 1359 & -1550 & 46 \\
\hline $\mathrm{H}(11)$ & -4753 & 1720 & -1046 & 55 \\
\hline $\mathrm{H}(12)$ & -3728 & 1322 & -901 & 56 \\
\hline $\mathrm{H}(13)$ & -3637 & 574 & -1341 & 45 \\
\hline $\mathrm{H}(15)$ & -2870 & -303 & -4539 & 34 \\
\hline $\mathrm{H}(16)$ & -1719 & -211 & -4742 & 46 \\
\hline $\mathrm{H}(17)$ & -1047 & 243 & -4009 & 51 \\
\hline $\mathrm{H}(18)$ & -1500 & 574 & -3010.9998 & 42 \\
\hline $\mathrm{H}(19)$ & -2654 & 479 & -2787 & 33 \\
\hline $\mathrm{H}(21)$ & -4742 & 359 & -4477 & 28 \\
\hline $\mathrm{H}(22)$ & -5223 & 1082 & -4692 & 32 \\
\hline $\mathrm{H}(23)$ & -4973 & 1689 & -3940 & 39 \\
\hline $\mathrm{H}(24)$ & -4241 & 1584 & -2970 & 38 \\
\hline $\mathrm{H}(25)$ & -3704 & 873 & -2788 & 34 \\
\hline $\mathrm{H}(27)$ & -2156 & -828 & -2892 & 39 \\
\hline $\mathrm{H}(28)$ & -1425 & -373.0000 & -2226 & 47 \\
\hline $\mathrm{H}(29)$ & -1536.0001 & -300 & -1029 & 53 \\
\hline $\mathrm{H}(30)$ & -2482 & -624 & -503.0000 & 46 \\
\hline $\mathrm{H}(31)$ & -3289 & -1017 & -1176 & 33 \\
\hline $\mathrm{H}(33)$ & -2321 & -1913 & -2737.9998 & 30 \\
\hline $\mathrm{H}(34)$ & -1534 & -2121 & -3574.9998 & 38 \\
\hline $\mathrm{H}(35)$ & -1630 & -1826.0001 & -4707 & 39 \\
\hline $\mathrm{H}(36)$ & -2488 & -1311 & -4998 & 44 \\
\hline $\mathrm{H}(37)$ & -3272 & -1092 & -4162 & 35 \\
\hline $\mathrm{H}(39)$ & -3161 & -1733 & -1258 & 38 \\
\hline $\mathrm{H}(40)$ & -3382 & -2460 & -802 & 48 \\
\hline $\mathrm{H}(41)$ & -3868 & -3022 & -1500.9999 & 48 \\
\hline $\mathrm{H}(42)$ & -4110 & -2872 & -2681 & 49 \\
\hline $\mathrm{H}(43)$ & -3950 & -2137 & -3127 & 43 \\
\hline $\mathrm{H}(45)$ & -6014.9995 & -1067 & -1985.0001 & 39 \\
\hline $\mathrm{H}(46)$ & -6504 & -1411 & -1016 & 50 \\
\hline $\mathrm{H}(47)$ & -6357 & -2191 & -818 & 56 \\
\hline $\mathrm{H}(48)$ & -5768 & -2631 & -1596 & 53 \\
\hline $\mathrm{H}(49)$ & -5303 & -2302 & -2602 & 44 \\
\hline $\mathrm{H}(51)$ & -4212 & -1531 & -4084 & 33 \\
\hline $\mathrm{H}(52)$ & -4034 & -2049 & -4984 & 44 \\
\hline $\mathrm{H}(53)$ & -4845 & -2613 & -5258 & 52 \\
\hline $\mathrm{H}(54)$ & -5850 & -2634 & -4640 & 48 \\
\hline $\mathrm{H}(55)$ & -6031 & -2116 & -3732 & 38 \\
\hline $\mathrm{H}(57)$ & -6743 & -1400 & -2992 & 41 \\
\hline $\mathrm{H}(58)$ & -7682 & -1062 & -3546.0002 & 52 \\
\hline $\mathrm{H}(59)$ & -7526.9995 & -533 & -4459 & 42 \\
\hline $\mathrm{H}(60)$ & -6426 & -334 & -4772 & 34 \\
\hline $\mathrm{H}(61)$ & -5497 & -675 & -4207 & 29 \\
\hline $\mathrm{H}(62)$ & -1631 & -4652 & -2356 & 25 \\
\hline $\mathrm{H}(65)$ & -637 & -3441.9998 & -1287 & 27 \\
\hline $\mathrm{H}(66)$ & -523 & -3606 & -100 & 33 \\
\hline $\mathrm{H}(67)$ & -431 & -4363 & 306 & 36 \\
\hline $\mathrm{H}(68)$ & -427 & -4966 & -507 & 31 \\
\hline $\mathrm{H}(70)$ & 491 & -5612 & -2302 & 81 \\
\hline $\mathrm{H}(71)$ & 651 & -6368 & -1949 & 94 \\
\hline $\mathrm{H}(72)$ & -133 & -6728 & -1263.0001 & 72 \\
\hline $\mathrm{H}(73)$ & -1080 & -6325 & -903 & 69 \\
\hline $\mathrm{H}(74)$ & -1252 & -5560 & -1249 & 50 \\
\hline $\mathrm{H}(76)$ & -89 & -5123 & -4204 & 34 \\
\hline $\mathrm{H}(77)$ & 475 & -5797 & -4519 & 47 \\
\hline $\mathrm{H}(78)$ & -32 & -6503.0005 & -4323 & 66 \\
\hline
\end{tabular}




\begin{tabular}{|c|c|c|c|c|}
\hline $\mathrm{H}(79)$ & -1089 & -6545 & -3798 & 74 \\
\hline $\mathrm{H}(80)$ & -1597.0001 & -5877 & -3406.0002 & 54 \\
\hline $\mathrm{H}(82)$ & -2570.9998 & -5131.0005 & -2737 & 40 \\
\hline $\mathrm{H}(83)$ & -3697.0002 & -5278 & -3112 & 48 \\
\hline $\mathrm{H}(84)$ & -3987.9998 & -5303 & -4283 & 51 \\
\hline $\mathrm{H}(85)$ & -3177 & -5150 & -5115 & 46 \\
\hline $\mathrm{H}(86)$ & -2066 & -4994 & -4767 & 34 \\
\hline $\mathrm{H}(88)$ & -2424 & -4154 & -3685 & 33 \\
\hline $\mathrm{H}(89)$ & -3147 & -3974 & -4615 & 41 \\
\hline $\mathrm{H}(90)$ & -3450 & -3210 & -4835 & 41 \\
\hline $\mathrm{H}(91)$ & -3008 & -2629 & -4117 & 46 \\
\hline $\mathrm{H}(92)$ & -2293 & -2815 & -3160.0002 & 35 \\
\hline $\mathrm{H}(94)$ & -2233 & -3281 & -1251 & 36 \\
\hline $\mathrm{H}(95)$ & -2087 & -2585 & -685 & 46 \\
\hline $\mathrm{H}(96)$ & -1458 & -1997 & -1171 & 45 \\
\hline $\mathrm{H}(97)$ & -923 & -2130 & -2234 & 39 \\
\hline $\mathrm{H}(98)$ & -1022 & -2846 & -2787 & 31 \\
\hline $\mathrm{H}\left(\begin{array}{llll}1 & 0 & 0\end{array}\right)$ & -1770 & -4302 & -1376 & 27 \\
\hline $\mathrm{H}(101)$ & -2606 & -4646 & -718 & 35 \\
\hline $\mathrm{H}(102)$ & -3749.0002 & -4500 & -970 & 37 \\
\hline $\mathrm{H}(1003)$ & -4049.0002 & -3999 & -1879.0001 & 38 \\
\hline $\mathrm{H}(104)$ & -3211 & -3664 & -2557 & 33 \\
\hline $\mathrm{H}(106)$ & -1102 & -3359 & -3931 & 35 \\
\hline $\mathrm{H}(107)$ & -1336 & -2864 & -4869 & 44 \\
\hline $\mathrm{H}(108)$ & -490 & -2374 & -5269 & 48 \\
\hline $\mathrm{H}(109)$ & 579 & -2391 & -4730 & 48 \\
\hline $\mathrm{H}(110)$ & 818 & -2882 & -3800 & 43 \\
\hline $\mathrm{H}(112)$ & 183 & -2565 & -2770 & 52 \\
\hline $\mathrm{H}(113)$ & 592 & -2106 & -1874 & 75 \\
\hline $\mathrm{H}(114)$ & 1119 & -2437 & -915 & 78 \\
\hline $\mathrm{H}(115)$ & 1167 & -3236 & -796 & 70 \\
\hline $\mathrm{H}(116)$ & 706 & -3709 & -1703.9999 & 53 \\
\hline $\mathrm{H}(118)$ & 1527 & -3553 & -2667 & 31 \\
\hline $\mathrm{H}(119)$ & 2478 & -3865.9998 & -3200 & 42 \\
\hline $\mathrm{H}(120)$ & 2351 & -4292 & -4229 & 46 \\
\hline $\mathrm{H}(121)$ & 1270 & -4424 & -4715 & 43 \\
\hline $\mathrm{H}(122)$ & 317 & -4120 & -4183 & 36 \\
\hline $\mathrm{H}(12 \mathrm{~A})$ & 2350 & -3718 & -533 & 56 \\
\hline $\mathrm{H}(12 \mathrm{~B})$ & 3070 & -3907.0002 & -776.9999 & 56 \\
\hline $\mathrm{H}(12 \mathrm{C})$ & -6723 & -1529 & -4795 & 63 \\
\hline $\mathrm{H}(12 \mathrm{D})$ & -6439 & -1812 & -5445 & 63 \\
\hline $\mathrm{H}(12 \mathrm{E})$ & -7498.0005 & -2199 & -3499 & 171 \\
\hline $\mathrm{H}(12 \mathrm{~F})$ & -8038 & -2577 & -3262 & 171 \\
\hline $\mathrm{H}(12 \mathrm{G})$ & -7100 & -2187 & -2360 & 129 \\
\hline $\mathrm{H}(12 \mathrm{H})$ & -7745 & -2495 & -2169 & 129 \\
\hline
\end{tabular}




\section{Theoretical data for the conversion of 2' in 3'.}

\section{Computational details}

All calculations were carried out using the Gaussian $03 \mathrm{~W}$ package. ${ }^{[3]}$ Geometry optimizations were carried out at the $\mathrm{B} 3 \mathrm{LYP}^{[4 \mathrm{a}]}$ level of theory, which is a hybrid functional consisting of Becke's exchange, ${ }^{[4 \mathrm{~b}]}$ Slater's exchange ${ }^{[4 \mathrm{c}]}$ exact Hartree-Fock exchange, VWN correlation, ${ }^{[4 \mathrm{~d}]}$ and the nonlocal (gradient) part of the LYP correlation ${ }^{[4 \mathrm{e}]}$ functionnals. The basis set LANL2DZ ${ }^{[4 f]}$ plus an f-type polarization function with an exponent of 0.400 was used for Ru. $(\mathrm{C}, \mathrm{H})$ atoms were represented by the $6-31 G^{*}$ basis set. $(P, S)$ atoms were represented by the $6-31+\mathrm{G}^{* *}$ basis set. 


\section{Theoretical data of 2':}

Figure S3-1: Molecular structure of 2'

View of the optimized structure Cartesian coordinates and energy computed at the B3LYP/[6-31+G*(H,C),6$\left.31+\mathrm{G}^{* *}(\mathrm{P}, \mathrm{S}), \mathrm{LANL} 2 \mathrm{DZ}+\mathrm{f}(\mathrm{Ru})\right]$ level of theory.

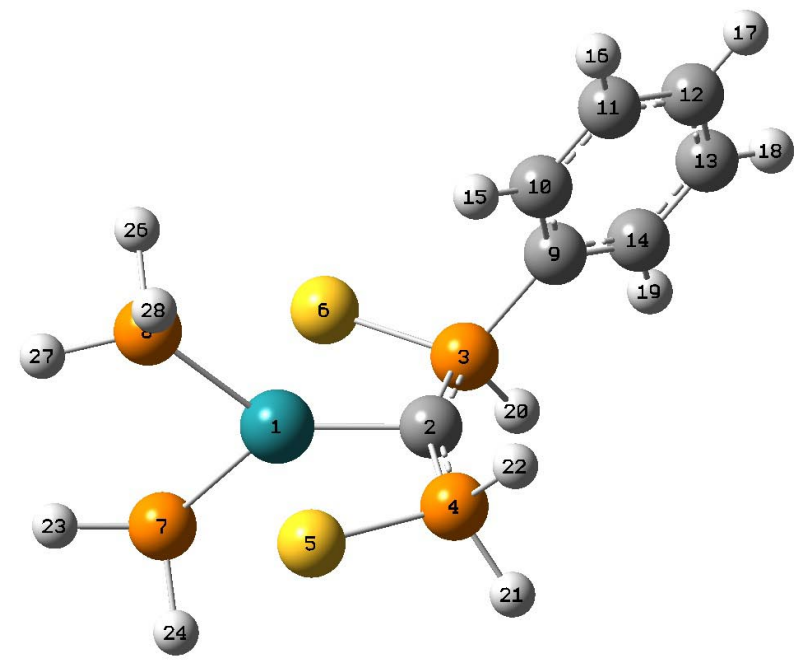


Table S3-2: Coordinates for 2'

Standard orientation:

\begin{tabular}{|c|c|c|c|c|c|}
\hline \multirow{2}{*}{$\begin{array}{l}\text { Center } \\
\text { Number }\end{array}$} & \multirow{2}{*}{$\begin{array}{l}\text { Atomic } \\
\text { Number }\end{array}$} & \multirow{2}{*}{$\begin{array}{c}\text { Atomic } \\
\text { Type }\end{array}$} & \multicolumn{3}{|c|}{ Coordinates (Angstroms) } \\
\hline & & & $\mathrm{X}$ & $\mathrm{Y}$ & $\mathrm{Z}$ \\
\hline-- & --- & $\ldots$ & ------- & -------- & ------- \\
\hline 1 & 44 & 0 & .033515 & -.065255 & -.004800 \\
\hline 2 & 6 & 0 & .025930 & -.063861 & 2.020476 \\
\hline 3 & 15 & 0 & 1.671718 & -.027990 & 2.416014 \\
\hline 4 & 15 & 0 & -1.613872 & -.252345 & 2.376878 \\
\hline 5 & 16 & 0 & -2.481112 & -.272169 & .520614 \\
\hline 6 & 16 & 0 & 2.512049 & .156293 & .549946 \\
\hline 7 & 15 & 0 & .186045 & -1.725263 & -1.554540 \\
\hline 8 & 15 & 0 & -.077777 & 1.496122 & -1.674983 \\
\hline 9 & 6 & 0 & 2.202457 & 1.241404 & 3.616628 \\
\hline 10 & 6 & 0 & 1.771193 & 2.569382 & 3.467072 \\
\hline 11 & 6 & 0 & 2.181720 & 3.543159 & 4.377485 \\
\hline 12 & 6 & 0 & 3.028184 & 3.200495 & 5.439396 \\
\hline 13 & 6 & 0 & 3.464240 & 1.881759 & 5.589338 \\
\hline 14 & 6 & 0 & 3.053297 & .903251 & 4.678593 \\
\hline 15 & 1 & 0 & 1.117742 & 2.832035 & 2.639013 \\
\hline 16 & 1 & 0 & 1.844992 & 4.569890 & 4.259795 \\
\hline 17 & 1 & 0 & 3.346938 & 3.961687 & 6.146890 \\
\hline 18 & 1 & 0 & 4.122444 & 1.613329 & 6.411617 \\
\hline 19 & 1 & 0 & 3.397027 & -.121898 & 4.799882 \\
\hline 20 & 1 & 0 & 2.157681 & -1.192567 & 3.067757 \\
\hline 21 & 1 & 0 & -1.956711 & -1.409779 & 3.130250 \\
\hline 22 & 1 & 0 & -2.181153 & .744981 & 3.218208 \\
\hline 23 & 1 & 0 & -.013880 & -1.593393 & -2.962625 \\
\hline 24 & 1 & 0 & -.683330 & -2.829137 & -1.370020 \\
\hline 25 & 1 & 0 & 1.408195 & -2.440343 & -1.624367 \\
\hline 26 & 1 & 0 & 1.024059 & 2.374989 & -1.832617 \\
\hline 27 & 1 & 0 & -.284885 & 1.241122 & -3.064783 \\
\hline 28 & 1 & 0 & -1.090673 & 2.478712 & -1.540677 \\
\hline
\end{tabular}




\section{Theoretical data of 2'TS4':}

\section{Figure S3-2: Molecular structure of 2'TS4'}

View of the optimized structure Cartesian coordinates, energy and the three lower frequencies computed at the B3LYP/[6-31+G*(H,C),6-31+G**(P,S),LANL2DZ+f(Ru)] level of theory.

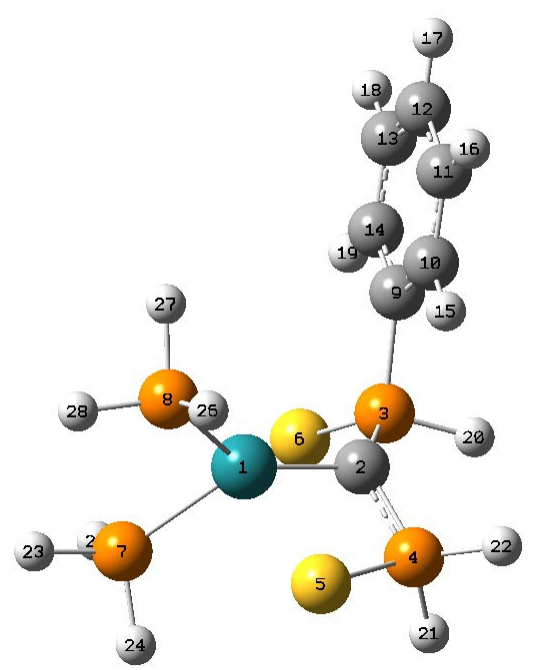


Table S3-2: Coordinates for 2'TS4'

Standard orientation:

\begin{tabular}{|c|c|c|c|c|c|}
\hline \multirow{2}{*}{$\begin{array}{l}\text { Center } \\
\text { Number }\end{array}$} & \multirow{2}{*}{$\begin{array}{l}\text { Atomic } \\
\text { Number }\end{array}$} & \multirow{2}{*}{$\begin{array}{l}\text { Atomic } \\
\text { Type }\end{array}$} & \multicolumn{3}{|c|}{ Coordinates (Angstroms) } \\
\hline & & & $\mathrm{X}$ & $\mathrm{Y}$ & Z \\
\hline-----1 & ------ & ------ & -------- & -------1 & ------- \\
\hline 1 & 44 & 0 & -.018103 & .034776 & -.009112 \\
\hline 2 & 6 & 0 & -.013213 & .029204 & 1.969081 \\
\hline 3 & 15 & 0 & 1.443944 & .007266 & 2.975677 \\
\hline 4 & 15 & 0 & -1.595869 & -.532026 & 2.330055 \\
\hline 5 & 16 & 0 & -2.375577 & -.479987 & .427527 \\
\hline 6 & 16 & 0 & 2.807064 & -1.375292 & 2.539656 \\
\hline 7 & 15 & 0 & .413667 & -1.780938 & -1.342798 \\
\hline 8 & 15 & 0 & -.346586 & 1.478916 & -1.826851 \\
\hline 9 & 6 & 0 & 2.127004 & 1.720416 & 2.984539 \\
\hline 10 & 6 & 0 & 1.276626 & 2.830978 & 2.873028 \\
\hline 11 & 6 & 0 & 1.797858 & 4.125901 & 2.941927 \\
\hline 12 & 6 & 0 & 3.171799 & 4.320249 & 3.117557 \\
\hline 13 & 6 & 0 & 4.022860 & 3.216086 & 3.222731 \\
\hline 14 & 6 & 0 & 3.503602 & 1.919661 & 3.156697 \\
\hline 15 & 1 & 0 & .212716 & 2.680448 & 2.712806 \\
\hline 16 & 1 & 0 & 1.132574 & 4.981280 & 2.851896 \\
\hline 17 & 1 & 0 & 3.577046 & 5.327946 & 3.167737 \\
\hline 18 & 1 & 0 & 5.092383 & 3.361895 & 3.352320 \\
\hline 19 & 1 & 0 & 4.161449 & 1.057352 & 3.224560 \\
\hline 20 & 1 & 0 & .979065 & -.108904 & 4.316325 \\
\hline 21 & 1 & 0 & -1.709617 & -1.825606 & 2.909131 \\
\hline 22 & 1 & 0 & -2.393160 & .207061 & 3.252433 \\
\hline 23 & 1 & 0 & .499087 & -1.839300 & -2.765100 \\
\hline 24 & 1 & 0 & -.457482 & -2.882530 & -1.171648 \\
\hline 25 & 1 & 0 & 1.643299 & -2.425783 & -1.064346 \\
\hline 26 & 1 & 0 & -1.606286 & 2.124550 & -1.879815 \\
\hline 27 & 1 & 0 & .459697 & 2.646841 & -1.862507 \\
\hline 28 & 1 & 0 & -.250586 & 1.195493 & -3.221555 \\
\hline
\end{tabular}

Sum of electronic and thermal Enthalpies=

$-2530.619172$

\begin{tabular}{lrrrr} 
& \multicolumn{1}{c}{1} & \multicolumn{1}{c}{2} & \multicolumn{1}{c}{3} \\
Frequencies -- & -33.2011 & \multicolumn{1}{c}{ A } & A \\
Red. masses -- & 9.8429 & 7.3739 & 15.4250 \\
Frc consts -- & .0064 & 3.8767 & 5.7578 \\
IR Inten & -- & 1.8897 & .0001 & .0008 \\
\end{tabular}




\section{Theoretical data of 4':}

Figure S3-3: Molecular structure of 4'

View of the optimized structure Cartesian coordinates and energy computed at the B3LYP/[6-31+G* $(H, C), 6-$ $\left.31+\mathrm{G}^{* *}(\mathrm{P}, \mathrm{S}), \mathrm{LANL} 2 \mathrm{DZ}+\mathrm{f}(\mathrm{Ru})\right]$ level of theory.

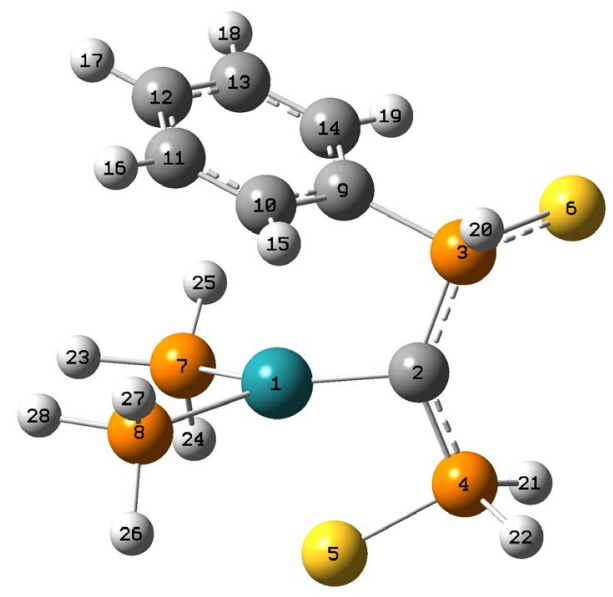


Table S3-3: Coordinates for 4'

Standard orientation:

\begin{tabular}{|c|c|c|c|c|c|}
\hline \multirow{2}{*}{$\begin{array}{l}\text { Center } \\
\text { Number }\end{array}$} & \multirow{2}{*}{$\begin{array}{l}\text { Atomic } \\
\text { Number }\end{array}$} & \multirow{2}{*}{$\begin{array}{c}\text { Atomic } \\
\text { Type }\end{array}$} & \multicolumn{3}{|c|}{ Coordinates (Angstroms) } \\
\hline & & & $\mathrm{X}$ & Y & $\mathrm{Z}$ \\
\hline \multicolumn{6}{|c|}{ 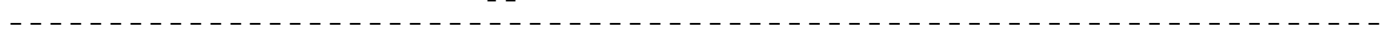 } \\
\hline 1 & 44 & 0 & .005949 & .001833 & .015312 \\
\hline 2 & 6 & 0 & .001803 & -.000063 & 1.987154 \\
\hline 3 & 15 & 0 & 1.585843 & -.023952 & 2.724176 \\
\hline 4 & 15 & 0 & -1.553567 & -.591692 & 2.390157 \\
\hline 5 & 16 & 0 & -2.354759 & -.670203 & .499037 \\
\hline 6 & 16 & 0 & 2.088352 & -1.450925 & 4.002168 \\
\hline 7 & 15 & 0 & .371847 & -1.625741 & -1.587599 \\
\hline 8 & 15 & 0 & -.532777 & 1.552246 & -1.627838 \\
\hline 9 & 6 & 0 & 2.553256 & .012009 & 1.143937 \\
\hline 10 & 6 & 0 & 2.417245 & 1.108843 & .263549 \\
\hline 11 & 6 & 0 & 3.155465 & 1.133050 & -.939115 \\
\hline 12 & 6 & 0 & 4.009950 & .083738 & -1.259087 \\
\hline 13 & 6 & 0 & 4.149826 & -1.001799 & -.375474 \\
\hline 14 & 6 & 0 & 3.429553 & -1.038989 & .814234 \\
\hline 15 & 1 & 0 & 1.865494 & 1.992662 & .573687 \\
\hline 16 & 1 & 0 & 3.069762 & 1.989946 & -1.603163 \\
\hline 17 & 1 & 0 & 4.584163 & .110967 & -2.181624 \\
\hline 18 & 1 & 0 & 4.836069 & -1.809926 & -.616477 \\
\hline 19 & 1 & 0 & 3.543296 & -1.864447 & 1.512522 \\
\hline 20 & 1 & 0 & 1.853546 & 1.266925 & 3.254167 \\
\hline 21 & 1 & 0 & -1.587827 & -1.842081 & 3.056854 \\
\hline 22 & 1 & 0 & -2.345844 & .192110 & 3.272333 \\
\hline 23 & 1 & 0 & .599696 & -1.440484 & -2.983964 \\
\hline 24 & 1 & 0 & -.616751 & -2.632420 & -1.705074 \\
\hline 25 & 1 & 0 & 1.491153 & -2.463781 & -1.356951 \\
\hline 26 & 1 & 0 & -1.909717 & 1.838279 & -1.795302 \\
\hline 27 & 1 & 0 & -.092604 & 2.886838 & -1.423820 \\
\hline 28 & 1 & 0 & -.199746 & 1.502584 & -3.015391 \\
\hline
\end{tabular}

Sum of electronic and thermal Enthalpies=

$-2530.630204$ 


\section{Theoretical data of 4'TS5':}

Figure S3-4: Molecular structure of 4'TS5'

View of the optimized structure Cartesian coordinates, energy and the three lower frequencies computed at the B3LYP/[6-31+G*(H,C),6-31+G**(P,S),LANL2DZ+f(Ru)] level of theory..

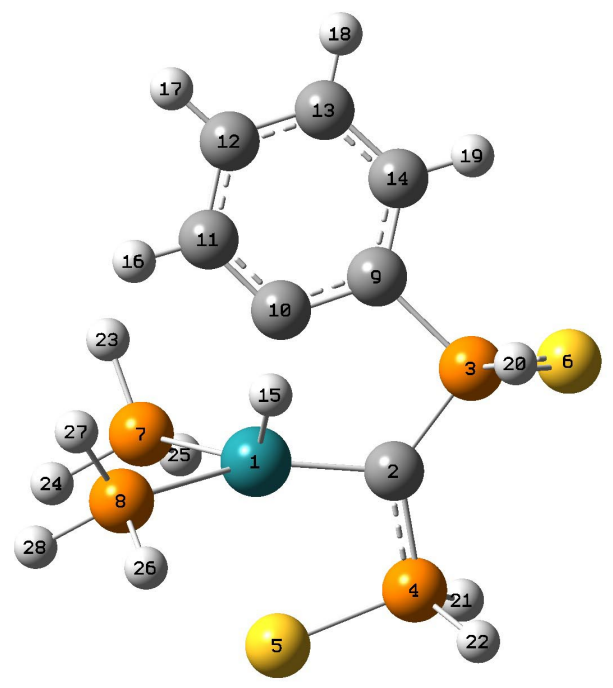




\section{Table S3-4: Coordinates for 4'TS5'}

Standard orientation:

\begin{tabular}{|c|c|c|c|c|c|}
\hline \multirow{2}{*}{$\begin{array}{l}\text { Center } \\
\text { Number }\end{array}$} & \multirow{2}{*}{$\begin{array}{l}\text { Atomic } \\
\text { Number }\end{array}$} & \multirow{2}{*}{$\begin{array}{l}\text { Atomic } \\
\text { Type }\end{array}$} & \multicolumn{3}{|c|}{ Coordinates (Angstroms) } \\
\hline & & & $\mathrm{X}$ & $\mathrm{Y}$ & $\mathrm{Z}$ \\
\hline-----1 & ----- & ------ & -------1 & -------- & ------- \\
\hline 1 & 44 & 0 & .000090 & -.008955 & .004489 \\
\hline 2 & 6 & 0 & .000125 & -.008168 & 1.926985 \\
\hline 3 & 15 & 0 & 1.494110 & -.004062 & 2.894598 \\
\hline 4 & 15 & 0 & -1.645696 & -.360839 & 2.367145 \\
\hline 5 & 16 & 0 & -2.550811 & -.497181 & .542117 \\
\hline 6 & 16 & 0 & 1.625880 & -1.317765 & 4.374003 \\
\hline 7 & 15 & 0 & .191677 & -1.920506 & -1.415755 \\
\hline 8 & 15 & 0 & -.613103 & 1.276960 & -1.950149 \\
\hline 9 & 6 & 0 & 2.672177 & -.120668 & 1.506759 \\
\hline 10 & 6 & 0 & 2.123548 & -.052243 & .204073 \\
\hline 11 & 6 & 0 & 3.032320 & -.067003 & -.869477 \\
\hline 12 & 6 & 0 & 4.408722 & -.208316 & -.662316 \\
\hline 13 & 6 & 0 & 4.922961 & -.322566 & .632755 \\
\hline 14 & 6 & 0 & 4.047351 & -.273066 & 1.719257 \\
\hline 15 & 1 & 0 & .992429 & 1.241133 & .011929 \\
\hline 16 & 1 & 0 & 2.665023 & .035090 & -1.888942 \\
\hline 17 & 1 & 0 & 5.080486 & -.227479 & -1.517960 \\
\hline 18 & 1 & 0 & 5.990802 & -.442952 & .794387 \\
\hline 19 & 1 & 0 & 4.427430 & -.368652 & 2.734204 \\
\hline 20 & 1 & 0 & 1.661899 & 1.326047 & 3.364648 \\
\hline 21 & 1 & 0 & -1.792130 & -1.525474 & 3.157255 \\
\hline 22 & 1 & 0 & -2.257155 & .608520 & 3.201466 \\
\hline 23 & 1 & 0 & 1.454215 & -2.284689 & -1.935281 \\
\hline 24 & 1 & 0 & -.588758 & -2.168055 & -2.577028 \\
\hline 25 & 1 & 0 & -.111433 & -3.119442 & -.733934 \\
\hline 26 & 1 & 0 & -1.471501 & 2.359654 & -1.655589 \\
\hline 27 & 1 & 0 & .334649 & 1.997882 & -2.718804 \\
\hline 28 & 1 & 0 & -1.357726 & .754189 & -3.041409 \\
\hline
\end{tabular}

\begin{tabular}{lrrrr} 
& \multicolumn{1}{c}{1} & \multicolumn{1}{c}{2} & \multicolumn{1}{c}{3} \\
Frequencies -- & -533.5532 & A & A \\
Red. masses -- & 1.1613 & 8.3725 & 60.3684 \\
Frc consts -- & .1948 & .0050 & 2.5431 \\
IR Inten & -- & 170.6398 & 1.3094 & .0055 \\
\end{tabular}




\section{Theoretical data of 5':}

Figure S3-5: Molecular structure of 5,

View of the optimized structure Cartesian coordinates and energy computed at the B3LYP/[6-31+G* $(\mathrm{H}, \mathrm{C}), 6-$ $\left.31+\mathrm{G}^{* *}(\mathrm{P}, \mathrm{S}), \mathrm{LANL} 2 \mathrm{DZ}+\mathrm{f}(\mathrm{Ru})\right]$ level of theory.

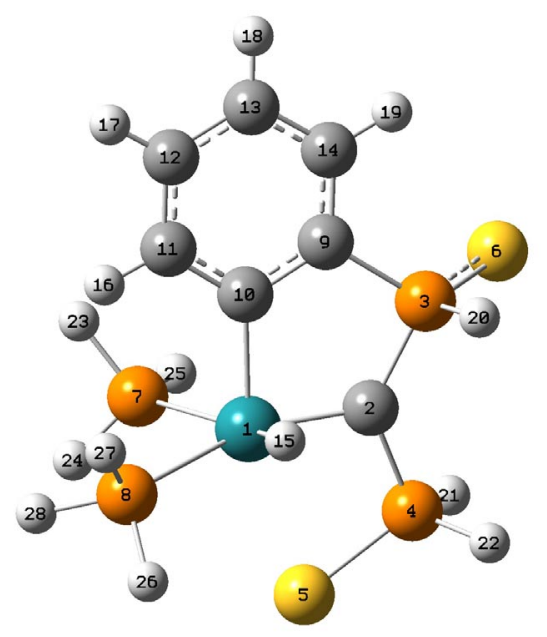


Table S3-5: Coordinates for 5'

Standard orientation:

\begin{tabular}{|c|c|c|c|c|c|}
\hline \multirow{2}{*}{$\begin{array}{l}\text { Center } \\
\text { Number }\end{array}$} & \multirow{2}{*}{$\begin{array}{l}\text { Atomic } \\
\text { Number }\end{array}$} & \multirow{2}{*}{$\begin{array}{l}\text { Atomic } \\
\text { Type }\end{array}$} & \multicolumn{3}{|c|}{ Coordinates (Angstroms) } \\
\hline & & & $\mathrm{X}$ & Y & Z \\
\hline \multicolumn{6}{|c|}{ - - - - - - - - - - - - - - - - - - - - - - - - - - - - - - - - - - - - - - - - - - - - - - - - - - - - - - - - - - - - - - - - - } \\
\hline 1 & 44 & 0 & .005923 & -.001096 & .003805 \\
\hline 2 & 6 & 0 & .004819 & .000032 & 1.881128 \\
\hline 3 & 15 & 0 & 1.533275 & -.006742 & 2.835165 \\
\hline 4 & 15 & 0 & -1.696350 & .070815 & 2.381603 \\
\hline 5 & 16 & 0 & -2.717238 & .089191 & .643280 \\
\hline 6 & 16 & 0 & 1.589002 & -1.306955 & 4.327839 \\
\hline 7 & 15 & 0 & -.090365 & -2.193619 & -1.059841 \\
\hline 8 & 15 & 0 & -.244392 & 1.097569 & -2.203010 \\
\hline 9 & 6 & 0 & 2.671263 & -.165226 & 1.428182 \\
\hline 10 & 6 & 0 & 2.070173 & -.145534 & .143965 \\
\hline 11 & 6 & 0 & 2.957097 & -.238095 & -.946876 \\
\hline 12 & 6 & 0 & 4.340659 & -.358508 & -.773417 \\
\hline 13 & 6 & 0 & 4.898219 & -.390796 & .509166 \\
\hline 14 & 6 & 0 & 4.054110 & -.293951 & 1.615778 \\
\hline 15 & 1 & 0 & .369987 & 1.579768 & .130384 \\
\hline 16 & 1 & 0 & 2.568255 & -.220334 & -1.964539 \\
\hline 17 & 1 & 0 & 4.986341 & -.430994 & -1.646612 \\
\hline 18 & 1 & 0 & 5.971798 & -.492310 & .643864 \\
\hline 19 & 1 & 0 & 4.465851 & -.332900 & 2.622346 \\
\hline 20 & 1 & 0 & 1.684714 & 1.333492 & 3.283447 \\
\hline 21 & 1 & 0 & -1.997417 & -1.001541 & 3.252864 \\
\hline 22 & 1 & 0 & -1.952531 & 1.180651 & 3.226278 \\
\hline 23 & 1 & 0 & 1.050207 & -2.680183 & -1.737813 \\
\hline 24 & 1 & 0 & -1.071184 & -2.555357 & -2.014678 \\
\hline 25 & 1 & 0 & -.278695 & -3.271520 & -.167816 \\
\hline 26 & 1 & 0 & -1.273837 & 2.063038 & -2.237536 \\
\hline 27 & 1 & 0 & .803435 & 1.909007 & -2.696267 \\
\hline 28 & 1 & 0 & -.560448 & .461419 & -3.434205 \\
\hline
\end{tabular}

Sum of electronic and thermal Enthalpies=

$-2530.616194$ 


\section{Theoretical data of 5'TS6':}

Figure S3-6: Molecular structure of 5'TS6'

View of the optimized structure Cartesian coordinates, energy and the three lower frequencies computed at the B3LYP/[6-31+G*(H,C),6-31+G**(P,S),LANL2DZ+f(Ru)] level of theory.

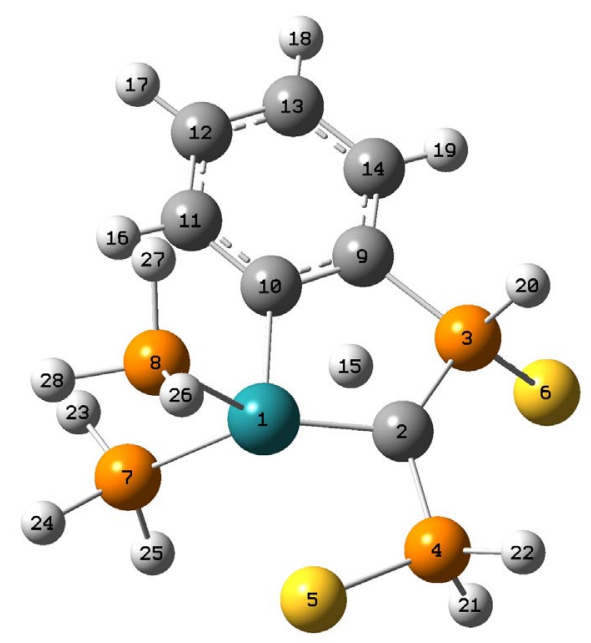




\section{Table S3-6: Coordinates for 5'TS6'}

\begin{tabular}{|c|c|c|c|c|c|}
\hline \multirow{2}{*}{$\begin{array}{l}\text { Center } \\
\text { Number }\end{array}$} & \multirow{2}{*}{$\begin{array}{l}\text { Atomic } \\
\text { Number }\end{array}$} & \multirow{2}{*}{$\begin{array}{c}\text { Atomic } \\
\text { Type }\end{array}$} & \multicolumn{3}{|c|}{ Coordinates (Angstroms) } \\
\hline & & & $\mathrm{X}$ & $\mathrm{Y}$ & $\mathrm{Z}$ \\
\hline---- & ---- & --- & -------- & -------- & ------- \\
\hline 1 & 44 & 0 & .002979 & .002132 & .002380 \\
\hline 2 & 6 & 0 & -.000632 & .009615 & 1.922084 \\
\hline 3 & 15 & 0 & 1.530525 & -.009412 & 2.859406 \\
\hline 4 & 15 & 0 & -1.701642 & -.121867 & 2.397508 \\
\hline 5 & 16 & 0 & -2.687489 & -.020186 & .638011 \\
\hline 6 & 16 & 0 & 1.572869 & -1.405049 & 4.261840 \\
\hline 7 & 15 & 0 & -.033202 & -1.875076 & -1.460814 \\
\hline 8 & 15 & 0 & -.113842 & 1.522025 & -1.819491 \\
\hline 9 & 6 & 0 & 2.670531 & -.060029 & 1.444189 \\
\hline 10 & 6 & 0 & 2.075024 & -.035811 & .154733 \\
\hline 11 & 6 & 0 & 2.978059 & -.040181 & -.928319 \\
\hline 12 & 6 & 0 & 4.364956 & -.083133 & -.747771 \\
\hline 13 & 6 & 0 & 4.914847 & -.122845 & .537582 \\
\hline 14 & 6 & 0 & 4.057969 & -.110359 & 1.638408 \\
\hline 15 & 1 & 0 & .148828 & 1.420730 & .893362 \\
\hline 16 & 1 & 0 & 2.598827 & -.013406 & -1.949645 \\
\hline 17 & 1 & 0 & 5.019266 & -.089241 & -1.617559 \\
\hline 18 & 1 & 0 & 5.991499 & -.165736 & .678841 \\
\hline 19 & 1 & 0 & 4.466127 & -.155290 & 2.646331 \\
\hline 20 & 1 & 0 & 1.693492 & 1.294393 & 3.403071 \\
\hline 21 & 1 & 0 & -1.837233 & -1.326687 & 3.119021 \\
\hline 22 & 1 & 0 & -2.147483 & .821862 & 3.361867 \\
\hline 23 & 1 & 0 & 1.172682 & -2.373150 & -2.007532 \\
\hline 24 & 1 & 0 & -.820703 & -1.925334 & -2.638065 \\
\hline 25 & 1 & 0 & -.504438 & -3.066264 & -.864856 \\
\hline 26 & 1 & 0 & -1.123258 & 2.504843 & -1.713222 \\
\hline 27 & 1 & 0 & .989068 & 2.381792 & -2.030830 \\
\hline 28 & 1 & 0 & -.338951 & 1.185497 & -3.182952 \\
\hline
\end{tabular}

\begin{tabular}{|c|c|c|c|}
\hline & 1 & 2 & 3 \\
\hline & A & A & A \\
\hline Frequencies & -750.3772 & 36.7917 & 56.5917 \\
\hline Red. masses & 1.1697 & 7.8626 & 1.0389 \\
\hline Frc consts & .3880 & .0063 & .0020 \\
\hline IR Inten & 33.7770 & 1.9796 & .1755 \\
\hline
\end{tabular}




\section{Theoretical data of 6':}

Figure S3-7: Molecular structure of 6'

View of the optimized structure Cartesian coordinates and energy computed at the B3LYP/[6-31+G* $(H, C), 6-$ $\left.31+\mathrm{G}^{* *}(\mathrm{P}, \mathrm{S}), \mathrm{LANL} 2 \mathrm{DZ}+\mathrm{f}(\mathrm{Ru})\right]$ level of theory.

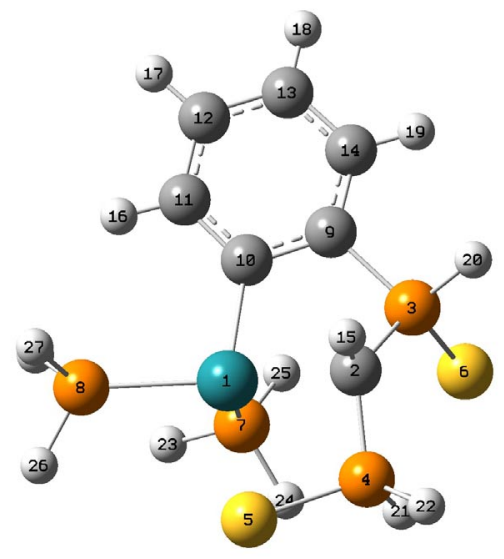


Table S3-7: Coordinates for 6'

Standard orientation:

\begin{tabular}{|c|c|c|c|c|c|}
\hline \multirow{2}{*}{$\begin{array}{l}\text { Center } \\
\text { Number }\end{array}$} & \multirow{2}{*}{$\begin{array}{l}\text { Atomic } \\
\text { Number }\end{array}$} & \multirow{2}{*}{$\begin{array}{c}\text { Atomic } \\
\text { Type }\end{array}$} & \multicolumn{3}{|c|}{ Coordinates (Angstroms) } \\
\hline & & & $\mathrm{X}$ & $\mathrm{Y}$ & Z \\
\hline 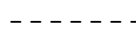 & & & --------- & -------- & -------- \\
\hline 1 & 44 & 0 & .001295 & .001336 & .002807 \\
\hline 2 & 6 & 0 & .000133 & .000783 & 2.183092 \\
\hline 3 & 15 & 0 & 1.719364 & -.001374 & 2.720681 \\
\hline 4 & 15 & 0 & -1.117083 & -1.365748 & 2.291699 \\
\hline 5 & 16 & 0 & -2.249605 & -1.224221 & .609404 \\
\hline 6 & 16 & 0 & 2.536074 & -1.804189 & 2.940228 \\
\hline 7 & 15 & 0 & 1.338372 & -1.698308 & -.530556 \\
\hline 8 & 15 & 0 & -.465720 & .400435 & -2.260031 \\
\hline 9 & 6 & 0 & 2.387667 & 1.130593 & 1.461808 \\
\hline 10 & 6 & 0 & 1.702791 & 1.168126 & .211936 \\
\hline 11 & 6 & 0 & 2.236959 & 2.063163 & -.740209 \\
\hline 12 & 6 & 0 & 3.346219 & 2.872877 & -.470855 \\
\hline 13 & 6 & 0 & 3.990190 & 2.810320 & .769100 \\
\hline 14 & 6 & 0 & 3.505834 & 1.930724 & 1.738083 \\
\hline 15 & 1 & 0 & -.499616 & .952564 & 2.410952 \\
\hline 16 & 1 & 0 & 1.778689 & 2.144182 & -1.724117 \\
\hline 17 & 1 & 0 & 3.711732 & 3.554090 & -1.236877 \\
\hline 18 & 1 & 0 & 4.855195 & 3.434866 & .976225 \\
\hline 19 & 1 & 0 & 4.000897 & 1.867358 & 2.705612 \\
\hline 20 & 1 & 0 & 1.819176 & .745531 & 3.923315 \\
\hline 21 & 1 & 0 & -.318533 & -2.521416 & 2.375395 \\
\hline 22 & 1 & 0 & -1.900597 & -1.468784 & 3.473759 \\
\hline 23 & 1 & 0 & 1.476641 & -2.035940 & -1.903380 \\
\hline 24 & 1 & 0 & 1.012878 & -2.984313 & -.040500 \\
\hline 25 & 1 & 0 & 2.702119 & -1.649232 & -.177880 \\
\hline 26 & 1 & 0 & -1.367970 & -.432836 & -2.969893 \\
\hline 27 & 1 & 0 & -1.093052 & 1.633251 & -2.574398 \\
\hline 28 & 1 & 0 & .528348 & .458267 & -3.274086 \\
\hline
\end{tabular}

Sum of electronic and thermal Enthalpies=

$-2530.667823$ 


\section{Theoretical data of 6'TS3':}

Figure S3-8: Molecular structure of 6'TS3'

View of the optimized structure Cartesian coordinates, energy and the three lower frequencies computed at the B3LYP/[6-31+G*(H,C),6-31+G**(P,S),LANL2DZ+f(Ru)] level of theory.

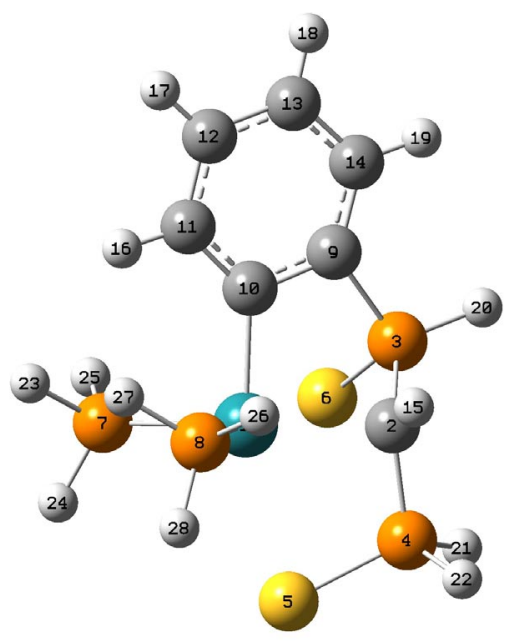




\section{Table S3-8: Coordinates for 6'TS3'}

\begin{tabular}{|c|c|c|c|c|c|}
\hline \multirow{2}{*}{$\begin{array}{l}\text { Center } \\
\text { Number }\end{array}$} & \multirow{2}{*}{$\begin{array}{l}\text { Atomic } \\
\text { Number }\end{array}$} & \multirow{2}{*}{$\begin{array}{c}\text { Atomic } \\
\text { Type }\end{array}$} & \multicolumn{3}{|c|}{ Coordinates (Angstroms) } \\
\hline & & & $\mathrm{X}$ & $\mathrm{Y}$ & $\mathrm{Z}$ \\
\hline----- & ---- & ---- & -------- & -------- & -------- \\
\hline 1 & 44 & 0 & -.000667 & .000352 & -.000161 \\
\hline 2 & 6 & 0 & -.000603 & -.001521 & 2.258265 \\
\hline 3 & 15 & 0 & 1.745840 & .002615 & 2.693194 \\
\hline 4 & 15 & 0 & -1.043746 & -1.417708 & 2.401683 \\
\hline 5 & 16 & 0 & -2.011119 & -1.572025 & .613985 \\
\hline 6 & 16 & 0 & 2.564473 & -1.805747 & 2.824849 \\
\hline 7 & 15 & 0 & .855076 & -1.144367 & -1.725335 \\
\hline 8 & 15 & 0 & -1.102178 & 1.299505 & -1.428413 \\
\hline 9 & 6 & 0 & 2.391255 & 1.132984 & 1.423126 \\
\hline 10 & 6 & 0 & 1.705257 & 1.169153 & .174338 \\
\hline 11 & 6 & 0 & 2.286856 & 1.998688 & -.808993 \\
\hline 12 & 6 & 0 & 3.440814 & 2.752321 & -.571573 \\
\hline 13 & 6 & 0 & 4.079969 & 2.701398 & .671779 \\
\hline 14 & 6 & 0 & 3.553378 & 1.879483 & 1.668387 \\
\hline 15 & 1 & 0 & -.512811 & .943325 & 2.469146 \\
\hline 16 & 1 & 0 & 1.833217 & 2.067625 & -1.796380 \\
\hline 17 & 1 & 0 & 3.844559 & 3.381783 & -1.362362 \\
\hline 18 & 1 & 0 & 4.976202 & 3.287247 & .858348 \\
\hline 19 & 1 & 0 & 4.052627 & 1.814122 & 2.633593 \\
\hline 20 & 1 & 0 & 1.921578 & .729122 & 3.900401 \\
\hline 21 & 1 & 0 & -.203488 & -2.506782 & 2.703974 \\
\hline 22 & 1 & 0 & -1.949603 & -1.409412 & 3.493949 \\
\hline 23 & 1 & 0 & 1.844497 & -.569172 & -2.562553 \\
\hline 24 & 1 & 0 & .052192 & -1.729824 & -2.740531 \\
\hline 25 & 1 & 0 & 1.559051 & -2.304399 & -1.326385 \\
\hline 26 & 1 & 0 & -2.054451 & 2.154001 & -.818931 \\
\hline 27 & 1 & 0 & -.441769 & 2.271514 & -2.223904 \\
\hline 28 & 1 & 0 & -1.944792 & .767208 & -2.441451 \\
\hline
\end{tabular}

\begin{tabular}{lrrrr} 
& & 1 & \multicolumn{1}{c}{2} & \multicolumn{1}{c}{3} \\
& & A & \multicolumn{1}{c}{ A } & A \\
Frequencies -- & -104.1978 & 30.3285 & 50.3809 \\
Red. masses -- & 9.7113 & 8.1835 & 1.0354 \\
Frc consts & -- & .0621 & .0044 & .0015 \\
IR Inten & -- & 1.7347 & 2.1646 & .2707
\end{tabular}




\section{Theoretical data of 3':}

Figure S3-9: Molecular structure of 3,

View of the optimized structure Cartesian coordinates and energy computed at the B3LYP/[6-31+G* $(H, C), 6-$ $\left.31+\mathrm{G}^{* *}(\mathrm{P}, \mathrm{S}), \mathrm{LANL} 2 \mathrm{DZ}+\mathrm{f}(\mathrm{Ru})\right]$ level of theory.

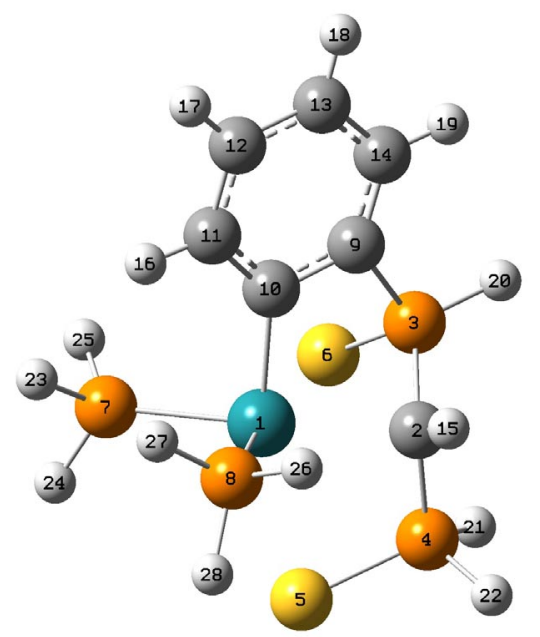


Table S3-9: Coordinates for 3'

Standard orientation:

\begin{tabular}{|c|c|c|c|c|c|}
\hline \multirow{2}{*}{$\begin{array}{l}\text { Center } \\
\text { Number }\end{array}$} & \multirow{2}{*}{$\begin{array}{l}\text { Atomic } \\
\text { Number }\end{array}$} & \multirow{2}{*}{$\begin{array}{c}\text { Atomic } \\
\text { Type }\end{array}$} & \multicolumn{3}{|c|}{ Coordinates (Angstroms) } \\
\hline & & & $\mathrm{X}$ & $\mathrm{Y}$ & Z \\
\hline \multicolumn{6}{|c|}{ 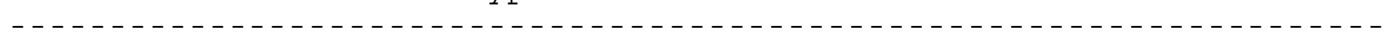 } \\
\hline 1 & 44 & 0 & .002049 & -.001312 & .003517 \\
\hline 2 & 6 & 0 & .000445 & -.000076 & 2.260212 \\
\hline 3 & 15 & 0 & 1.774574 & -.001824 & 2.069699 \\
\hline 4 & 15 & 0 & -.894291 & -1.542581 & 2.392927 \\
\hline 5 & 16 & 0 & -1.673863 & -2.074399 & .615930 \\
\hline 6 & 16 & 0 & 2.052971 & -1.502844 & .731729 \\
\hline 7 & 15 & 0 & .326166 & -.303741 & -2.277244 \\
\hline 8 & 15 & 0 & -1.758333 & 1.415551 & -.215333 \\
\hline 9 & 6 & 0 & 2.098654 & 1.573644 & 1.276856 \\
\hline 10 & 6 & 0 & 1.273718 & 1.631143 & .121432 \\
\hline 11 & 6 & 0 & 1.444774 & 2.771601 & -.686539 \\
\hline 12 & 6 & 0 & 2.367729 & 3.771437 & -.351245 \\
\hline 13 & 6 & 0 & 3.161238 & 3.674393 & .799655 \\
\hline 14 & 6 & 0 & 3.031052 & 2.555003 & 1.625938 \\
\hline 15 & 1 & 0 & -.446223 & .849432 & 2.780124 \\
\hline 16 & 1 & 0 & .856500 & 2.894632 & -1.595109 \\
\hline 17 & 1 & 0 & 2.473346 & 4.639348 & -.999648 \\
\hline 18 & 1 & 0 & 3.873393 & 4.457915 & 1.044468 \\
\hline 19 & 1 & 0 & 3.647840 & 2.453324 & 2.517306 \\
\hline 20 & 1 & 0 & 2.585902 & -.094754 & 3.238720 \\
\hline 21 & 1 & 0 & -.075304 & -2.538000 & 2.968776 \\
\hline 22 & 1 & 0 & -1.869195 & -1.377279 & 3.407615 \\
\hline 23 & 1 & 0 & .115882 & .751323 & -3.206167 \\
\hline 24 & 1 & 0 & -.379646 & -1.298911 & -3.002366 \\
\hline 25 & 1 & 0 & 1.626831 & -.653511 & -2.712138 \\
\hline 26 & 1 & 0 & -2.056808 & 2.290272 & .861214 \\
\hline 27 & 1 & 0 & -1.779309 & 2.398661 & -1.239806 \\
\hline 28 & 1 & 0 & -3.068434 & .911250 & -.413935 \\
\hline--- & & - & ---------- & -------- & --------- \\
\hline of & $i^{2}$ & & 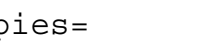 & -2530 & 099 \\
\hline
\end{tabular}




\section{References:}

[1] A. Altomare, M. C. Burla, M. Camalli, G. Cascarano, C. Giacovazzo, A. Guagliardi, A. G. G. Moliterni, G. Polidori, R. Spagna, SIR97, an integrated package of computer programs for the solution and refinement of crystal structures using single crystal data.

[2] G. M. Sheldrick, SHELXL-97, Universität Göttingen, Göttingen, Germany, 1997.

[3] Gaussian 03, Revision B.04, M. J. Frisch, G. W. Trucks, H. B. Schlegel, G. E. Scuseria, M. A. Robb, J. R. Cheeseman, J. A. Montgomery, Jr., T. Vreven, K. N. Kudin, J. C. Burant, J. M. Millam, S. S. Iyengar, J.

Tomasi, V. Barone, B. Mennucci, M. Cossi, G. Scalmani, N. Rega, G. A. Petersson, H. Nakatsuji, M. Hada, M. Ehara, K. Toyota, R. Fukuda, J. Hasegawa, M. Ishida, T. Nakajima, Y. Honda, O. Kitao, H. Nakai, M. Klene, X. Li, J. E. Knox, H. P. Hratchian, J. B. Cross, V. Bakken, C. Adamo, J. Jaramillo, R. Gomperts, R. E. Stratmann, O. Yazyev, A. J. Austin, R. Cammi, C. Pomelli, J. W. Ochterski, P. Y. Ayala, K. Morokuma, G. A. Voth, P. Salvador, J. J. Dannenberg, V. G. Zakrzewski, S. Dapprich, A. D. Daniels, M. C. Strain, O. Farkas, D. K. Malick, A. D. Rabuck, K. Raghavachari, J. B. Foresman, J. V. Ortiz, Q. Cui, A. G. Baboul, S. Clifford, J. Cioslowski, B. B. Stefanov, G. Liu, A. Liashenko, P. Piskorz, I. Komaromi, R. L. Martin, D. J. Fox, T. Keith, M. A. Al-Laham, C. Y. Peng, A. Nanayakkara, M. Challacombe, P. M. W. Gill, B. Johnson, W. Chen, M. W. Wong, C. Gonzalez, and J. A. Pople, Gaussian, Inc., Wallingford CT, 2004.

[4] a) A. D. Becke, J. Chem. Phys., 1993, 98, 5648; b) A. D. Becke, Phys. Rev. A, 1998, 38, 3098; c) P. Hohenberg, W. Kohn, Phys. Rev. B, 1964, 136, 864; W. Kohn, L. J. Sham, Phys. Rev. A, 1965, 140, 1133; J. C. Slater In Quantum Theory of Molecular and Solids Vol. 4 : The Self-Consistent Field for Molecular and Solids, McGraw-Hill, New York, 1974; d) S H. Vosko, L. Wilk, M. Nusair, Can. J. Phys., 1980, 58, 1200; e) C. Lee, W. Yang, R.G. Parr, Phys. Rev. B, 1988, 37, 785; B. Miehlich, A. Savin, H. Stoll, H. Preuss, Chem. Phys. Lett., 1989, 157, 200; f) Hay, P. J.; Wadt, W. R. J. Chem. Phys., 1985, 82, 299. 